Florida International University

FIU Digital Commons

8-29-2018

\title{
An Investigation into the Adduct Forming Potential of Drugs of Abuse with Peptides and Proteins
}

Richard Allen Gilliland

Florida International University, rgill024@fiu.edu

Follow this and additional works at: https://digitalcommons.fiu.edu/etd

Part of the Other Chemistry Commons

\section{Recommended Citation}

Gilliland, Richard Allen, "An Investigation into the Adduct Forming Potential of Drugs of Abuse with Peptides and Proteins" (2018). FIU Electronic Theses and Dissertations. 3869.

https://digitalcommons.fiu.edu/etd/3869

This work is brought to you for free and open access by the University Graduate School at FIU Digital Commons. It has been accepted for inclusion in FIU Electronic Theses and Dissertations by an authorized administrator of FIU Digital Commons. For more information, please contact dcc@fiu.edu. 


\title{
FLORIDA INTERNATIONAL UNIVERSITY \\ Miami, Florida
}

\section{AN INVESTIGATION INTO THE ADDUCT FORMING POTENTIAL OF DRUGS OF ABUSE WITH PEPTIDES AND PROTEINS}

\author{
A dissertation submitted in partial fulfillment of \\ the requirements for the degree of \\ DOCTOR OF PHILOSOPHY \\ in \\ CHEMISTRY \\ by
}

Richard Allen Gilliland

2018 
To: Dean Michael R. Heithaus

College of Arts, Sciences, and Education

This dissertation, written by Richard Allen Gilliland, and entitled An Investigation into the Adduct Forming Potential of Drugs of Abuse with Peptides and Proteins, having been approved in respect to style and intellectual content, is referred to you for judgment.

We have read this dissertation and recommend that it be approved.

$\begin{array}{r}\text { Deitrich Lorke } \\ \hline \text { Bruce McCord } \\ \hline \text { Yi Xiao } \\ \hline \text { Yuk-Ching Tse-Dinh } \\ \hline \text { Anthony DeCaprio, Major Professor }\end{array}$

Date of Defense: August 29, 2018

The dissertation of Richard Allen Gilliland is approved.

Dean Michael R. Heithaus
College of Arts, Science, and Education

College of Arts, Science, and Education

Andrés G. Gil

Vice President for Research and Economic Development and Dean of the University Graduate School

Florida International University, 2018 
(C) Copyright 2018 by Richard Allen Gilliland

All rights reserved by the author with the exception of Figures 6 and 7. Figures 6 and 7 have been included with the permission of the respective publishers. 


\section{DEDICATION}

This is dedicated to my friends and family who have helped me not only through my time in graduate school but also through life. I should hope to someday be able to impart the wisdom, strength, forgiveness, love, and generosity which you have all shown to me.

"I do not feel obliged to believe that the same God who has endowed us with senses, reason, and intellect has intended us to forego their use..."

- Galileo Galilei 


\section{ACKNOWLEDGMENTS}

I would like to first acknowledge my advisor, Dr. Anthony DeCaprio. You have been able to challenge me to achieve so much in my time at FIU, and you have been there to provide support, feedback, and ideas when I was in need of any of the three. I would like to thank my committee members, Dr. Bruce McCord, Dr. Dietrich Lorke, Dr. Yi Xiao, and Dr. Yuk-Ching Tse-Dinh, for sitting through all of my presentations, reading my papers, and ensuring that I was on the right track for my research by providing suggestions and posing questions.

I would like to thank the National Institute of Justice for funding provided to my research, allowing me to travel to conferences and present my research on local, regional, and national scales. I would also like to thank the FIU Department of Chemistry for providing funding through a teaching assistantship and Dr. Graves for being a wonderful mentor through my teaching experiences.

The transition to graduate school was a difficult one, and one that I would not have been able to make were it not for several DeCaprio lab alumni, including Dr. Vanessa Quinn and Dr. Kevin Schneider. Tips and advice that may have seemed insignificant to you made a world of difference to me as I was starting out. Thank you for both being such meticulous note takers, record keepers, and super scientists.

To my fellow DeCaprio lab mates, l'd like to say thank you for keeping me focused, on track, accountable, and most importantly sane. Dr. Melanie Eckberg, Jenna Chenevert, and Ashley Kimble, you have all been there for times when I had questions, concerns, needed to discuss ideas, or needed anything 
proofread. The rigors of graduate school are hard, but they would have been much worse had I not been surrounded by people like you every day. Much of what I've been able to accomplish in graduate school was because of the help of Dr. Carolina Moller. You have taught me more about peptides, proteins, and their respective analyses, than I could have ever hoped. Thank you for taking your time and expending the effort into making sure I knew what I needed to know. To William Morrison and Ludmyla Tavares, I say thank you for continuing this project and furthering the research which I started. I wish you both the best of luck.

Lastly, I would like to say thank you to my friends, both at FIU and not, and family. To Quentin Gauthier, Dr. Alison Simon, and Dr. Peter Regier, thank you for broadening my interests and knowledge by introducing me to topics, methods, and fields of research that I knew nothing about. To my family, thank you for your endless love and support. My siblings, Michael and Rebecca, and my parents, Richard and Melissa, have been instrumental in me finding the courage and fortitude to continue through the many hardships of graduate school and even the decision to pursue a graduate degree in the first place. To my fiancée Mariah Martinez, thank you for your never ending love, patience, and understanding. You have pushed me to achieve milestones throughout this process and been there to celebrate along the way. 


\section{ABSTRACT OF THE DISSERTATION \\ AN INVESTIGATION INTO THE ADDUCT FORMING POTENTIAL OF DRUGS \\ OF ABUSE WITH PEPTIDES AND PROTEINS}

by

Richard Allen Gilliland

Florida International University, 2018

Miami, Florida

Professor Anthony DeCaprio, Major Professor

Hemoglobin and serum albumin, two prevalent proteins in human blood, contain unbound cysteine thiol moieties, creating a nucleophilic site with the potential for covalent modification by reactive chemical species. These covalent modifications, called "adducts", are stable entities that accumulate during acute and chronic exposure and remain covalently bound for the life-span of the protein. Despite their current use as exposure markers for a variety of compounds, the use of adducts in assessing exposure to drugs of abuse has not yet been explored. The goal of this work was to examine the in vitro adduct forming capability of selected drugs of abuse with hemoglobin to provide additional proof of principle for the development of a real-world detection and monitoring analysis method. This goal was accomplished by first analyzing the binding capabilities of the drugs of interest with glutathione, a smaller tripeptide. Use of protein adducts as biomarkers of drug exposure may allow for an increased window of detection, from several days to several months, as compared to current blood analysis methods. In total, there were 16 drugs 
analyzed in the research, and they covered a wide range of abused drugs, including cocaine, methamphetamine, and $\Delta^{9}-\mathrm{THC}$.

Results from the glutathione trials showed that 10 of the 16 the drugs of interest were able to form covalent adducts with the free thiol moiety, with four drugs forming more than one novel adduct. The MS results for hemoglobin showed 11 adducts formed for five of the drugs under investigation. Additional MS/MS confirmatory data was obtained for two of those 11 adducts. I successfully identifyied adducts formed between drugs of abuse and glutathione and hemoglobin, which have the potential to be used as long-term biomarkers of exposure. 


\section{TABLE OF CONTENTS}

CHAPTER

PAGE

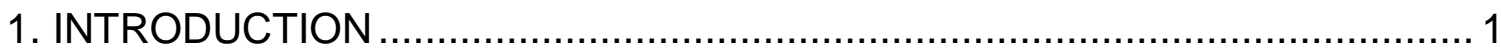

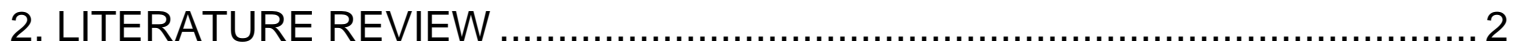

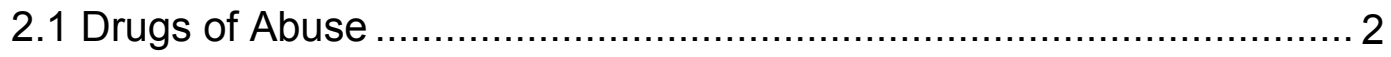

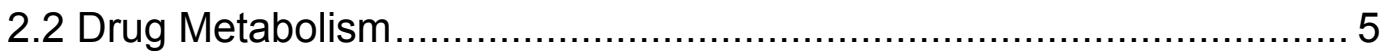

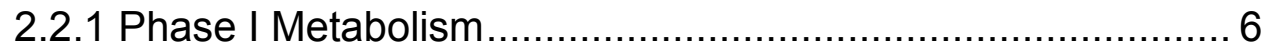

2.2.2 Phase II Metabolism.................................................... 9

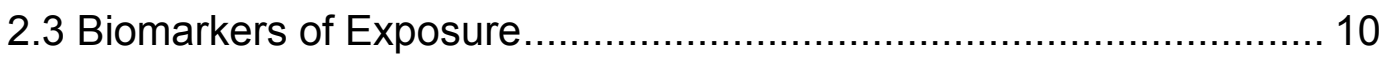

2.3.1 Blood and Urine as a Matrix ............................................ 10

2.3.2 Hair as a Matrix ................................................................. 11

2.4 Use of Protein Adducts as Exposure Biomarkers ........................... 13

2.4.1 Protein Adduction by Drugs of Abuse ……....................... 14

2.4.2 Hard-Soft Acid-Base Theory ......................................... 16

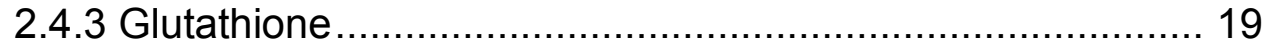

2.4.4 Hemoglobin as a Target for Reactive Xenobiotics ............... 21

2.5 LC-MS Analysis of Covalent Protein Modifications ........................... 24

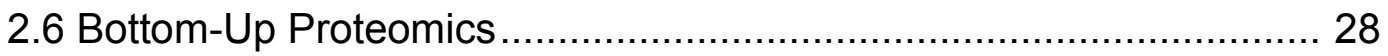

2.6.1 LC-MS Peptide and Protein Analysis ............................... 30

2.7 In Vitro Metabolic Assays............................................................. 31

2.7.1 In Vitro Metabolic Trapping Assays ................................... 31

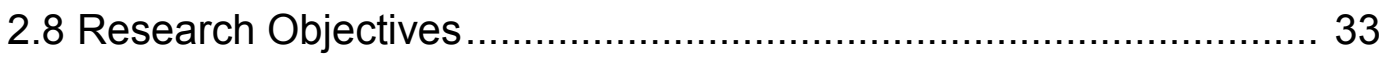

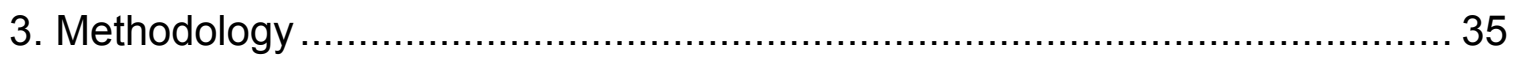

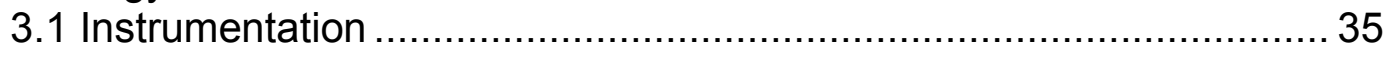

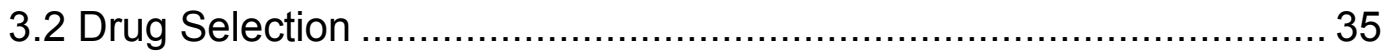

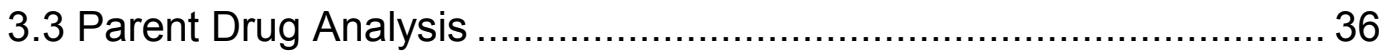

3.4 Drugs of Abuse Stable Metabolites ................................................ 37

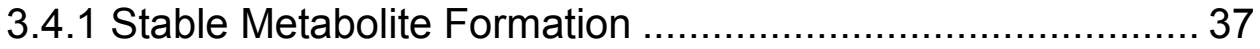

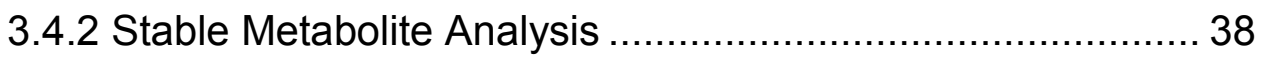

3.5 Metabolic Trapping Assay with Glutathione ................................... 39

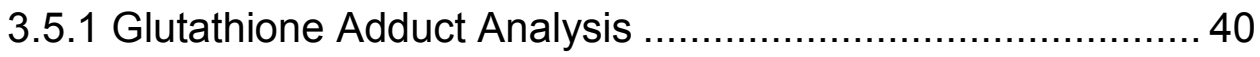

3.5.2 Glutathione Adduct Confirmation ...................................... 41

3.6 Identification of Glutathione Adduct Structures ............................... 42

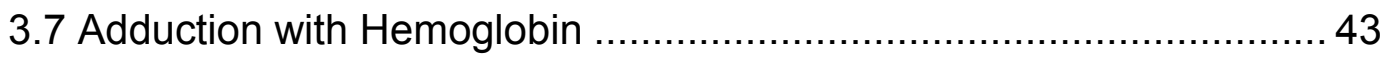

3.7.1 Hemoglobin Trapping Assay Method ............................... 43

3.7.1 Hemoglobin Adduct Analysis........................................... 45 
4. Results

4.1 Task 1: Create and optimize a LC-MS/MS method for the detection and characterization of drugs of interest and stable drug metabolites. .... 47

4.1.1 Parent Drug Retention Times ............................................ 47

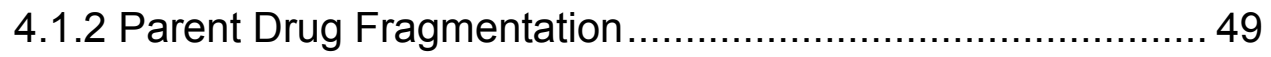

4.1.3 Stable Drug Metabolites ................................................ 58

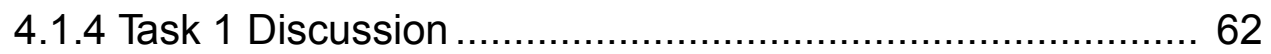

4.2 Task 2: Create and optimize a LC-MS/MS method for the detection and characterization of reactive drug metabolites....................................6 63

4.2.1 Metabolic Trapping Assay Optimization ............................. 63

4.2.2 MS and MS/MS Analysis of Glutathione Adducts................. 64

4.2.3 Glutathione Adduct Structure Identification ......................... 77

4.2.4 Task 2 Discussion ..................................................... 83

4.3 Confirmation of adduction in human $\mathrm{Hb}$ by drugs of interest ............ 87

4.3.1 Protein Adduction Assay Optimization .............................. 87

4.3.2 Analysis of Hemoglobin Trapping Assay Products .............. 88

4.3.3 MS/MS Analysis of Hemoglobin Adducts .......................... 91

4.3.4 Task 3 Discussion ....................................................... 96

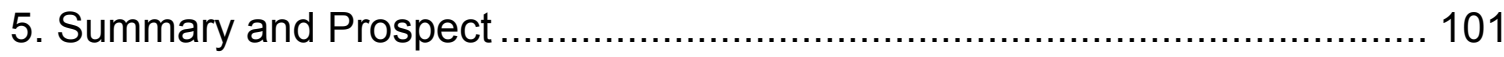

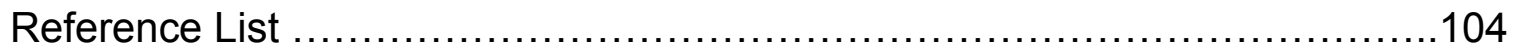

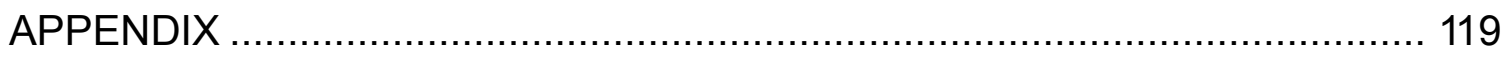

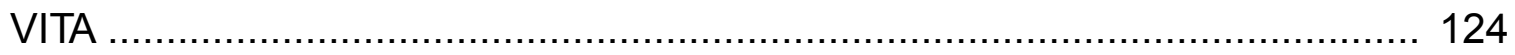




\section{LIST OF TABLES}

TABLE

PAGE

Table 1. Drug schedules as determined by the U.S. DEA ............................... 3

Table 2. The drugs of interest selected for this study in addition to the abbreviations used for each. Table 2 also lists the formula, US DEA schedule, and general class of each drug of interest.

Table 3. Some of the chemical groups which may be metabolized by CYP enzymes and the reactions possible with each.

Table 4. Phase II metabolic processes and the respective enzymes and cofactors required for each ......................................................................

Table 5. A summary of the retention times in blood and urine for methamphetamine, MDMA, cannabis, and heroin, as reported in hours. 11

Table 6. Mixtures of parent drugs created to collect transitions

Table 7. Retention time and fragmentation data collected for all 16 drugs of interest in this research. Retention time is in minutes. Transitions collected were the three most prominent across all collision energies..... 58

Table 8. Theoretical adduct masses collected via QQQ-MS/MS and QTOF MS/MS.

Table 9. Summary of fragmentation of all GSH adducts observed. ................... 76

Table 10. Summary of MS data collected for $\mathrm{Hb}$ adducts. The asterisk for adduct 11 indicates a missed tryptic cleavage in the identified adduct. ...91

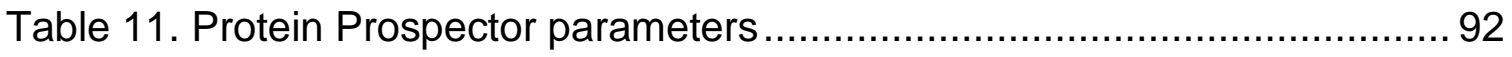

Table 12. Summary of MS/MS peak information for $\mathrm{Hb}+\mathrm{APAP}$ adduct.

Possible match peaks are indicated with an asterisk.

Table 13. Summary of MS/MS peak information for $\mathrm{Hb}+\mathrm{a}-\mathrm{PVP}$ adduct. Possible match peaks are indicated with an asterisk. 


\section{LIST OF FIGURES}

FIGURE

PAGE

Figure 1 . The process of heme cycling by a CYP enzyme to produce an oxidized substrate. 8

Figure 2. The window of detection for cocaine and its metabolites in blood, urine, and hair set to a logarithmic scale. ${ }^{35}$

Figure 3. The chemical structure of glutathione showing the appropriate charges on the terminal carboxy groups and the amine group.

Figure 4. The porphyrin ring system found in the heme B iron complex of hemoglobin.

Figure 5. This diagram shows the four major scanning modes for an LC-QqQMS system.

Figure 6. Schematic for a generic QTOF instrument, showing the quadrupole for ion selection $\left(Q_{1}\right)$, the collision cell $\left(q_{2}\right)$, orthogonal accelerator, reflectron, and detector. ${ }^{112}$ The gas used in the collision cell for this research was $\mathrm{N}_{2}$

Figure 7. This diagram shows the general procedures for top-down, middledown, and bottom-up proteomics. ${ }^{113}$

Figure 8. Peptide fragmentation patterns labeled with the common "a,b,c" and " $\mathrm{x}, \mathrm{y}, \mathrm{z}$ " nomenclature.

Figure 9. Illustration of the metabolic trapping assay method utilizing GSH as a trapping agent.

Figure 10. Illustration of the metabolic trapping assay method utilizing $\mathrm{Hb}$ as a trapping agent.

Figure 11. Flowchart of workflow for $\mathrm{Hb}$ peptide adduct analysis. 46

Figure 12. A zoomed in portion of the overlaid TICs for a-PVP, ALP, BUP, COC, DZP, MDMA, MDPV, META, METH, METY, MOR, NAL and OXY 48

Figure 13. TICs for CLZ (top) and APAP (bottom), the two positive controls, used to collect their respective retention times.

Figure 14. TIC (top) and XIC (bottom) for THC, showing the retention time for $\mathrm{THC}$ 
Figure 15. Targeted MS/MS TICs for the five mixtures of the parent drugs. 52

Figure 16. MS/MS data for the parent drugs. The data are shown at $10 \mathrm{eV}$ (top), $20 \mathrm{eV}$ (middle), and $40 \mathrm{eV}$ (bottom) for each drug.

Figure 17. Two major metabolites for clozapine, clozapine N-oxide (left) and Ndesmethylclozapine (right) with their exact masses.

Figure 18. The TICs for the in vitro metabolic assay for clozapine (top) and the negative control with no metabolic system (bottom). The two new peaks are labeled with their corresponding metabolite.

Figure 19. XIC data for $\mathrm{N}$-desmethylclozapine (top) and clozapine $\mathrm{N}$-oxide (bottom).

Figure 20 The TICs for the in vitro metabolic assay for COC; the two new peaks are labeled with their corresponding metabolite.

Figure $21 \mathrm{XIC}$ data for cocaethylene (top) and benzoylecgonine (bottom).

Figure 22. (A) TIC of APAP+GSH collected by product ion scan mode via LCQqQ-MS in negative ionization mode. (B) XICs for ions at $m / z 471$ and 455 , the two relevant peaks of interest from the TIC.

Figure 23. LC-QTOF-MS/MS data collected for glutathione. The peaks labeled all correspond to a known characteristic GSH peak. Negative ionization structures for each peak are provided for each peak.

Figure 24. Detailed breakdown of the nomenclature used for the fragmentation of the GSH adducts. Data presented are from Xie et al. ${ }^{88}$.

Figure 25. MS/MS spectra for all 12 previously unreported adducts. Proposed structures for 9 of the 12 adducts are also shown with GSH linkage indicated.

Figure 26. MS/MS data collected for all previously reported GSH adducts ........ 74

Figure 27. MS/MS data collected for APAP1 and APAP2 ............................. 78

Figure 28. TIC (a) and TCC (b) for the APAP $+\mathrm{Hb}$ adduct. The removal of certain peaks was accomplished using the BioConfirm software............. 89

Figure 29. BioConfirm method parameters...................................................... 90

Figure 30. The BioConfirm sample processing method utilized for the adducted

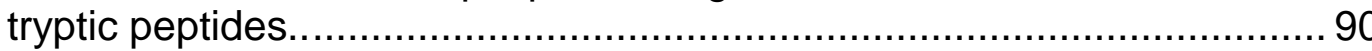


Figure 31. MS/MS identification of the adduct formed between APAP and $\mathrm{Hb}$

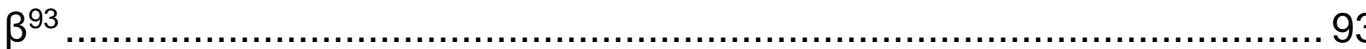

Figure 32. MS/MS data for the unmodified peptide containing the $\mathrm{Hb} \beta^{93}$

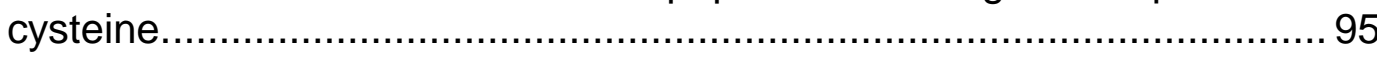

Figure 33. MS/MS identification of the adduct formed between $\alpha$-PVP and $\mathrm{Hb}$

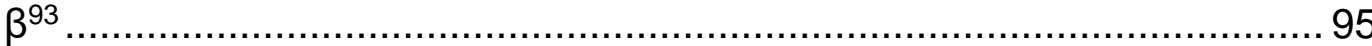

Figure 34. Example of custom modification settings used for BioConfirm analysis

Figure 35. Space-filled model of $\mathrm{Hb}$ with $\mathrm{Hb} \beta^{93}$ highlighted in green................ 99

Figure 36. Space-filled model of $\mathrm{Hb}$ with $\mathrm{His}$ and Lys residues highlighted in green 99 


\section{LIST OF ABBREVIATIONS}

\begin{tabular}{ll} 
ADME & Adsorption, distribution, metabolism, excretion \\
ALP & Alprazolam \\
APAP & Acetaminophen \\
BSA & Bovine serum albumin \\
BUP & Buprenorphine \\
CID & Collision induced dissociation \\
CLZ & Clozapine \\
CNS & Central nervous system \\
COC & Cocaine \\
CYP & Cytochrome P450 enzyme \\
Cys & Cysteine \\
DEA & Drug Enforcement Administration \\
DZP & Diazepam \\
ESI & Electrospray ionization \\
FMO & Frontier molecular orbitals \\
G6P & Glucose-6-phosphate \\
G6PD & Glucose-6-phosphate dehydrogenase \\
GSH & Glutathione \\
GST & Glutathione-s-transferase \\
Hb & Hemoglobin \\
Hb a & Hemoglobin a subunit \\
\hline
\end{tabular}




\begin{tabular}{ll} 
Hb $\beta$ & Hemoglobin $\beta$ subunit \\
Histidine & His \\
HLM & Human liver microsomes \\
HOMO & Highest occupied molecular orbital \\
HSA & Human serum albumin \\
HSAB & Hard and soft acids and bases theory \\
IAM & lodoacetamide \\
LC & Liquid chromatography \\
LUMO & Lowest unoccupied molecular orbital \\
Lysine & Lys \\
m/z & Mass-to-charge ratio \\
MDMA & Methylenedioxymethamphetamine \\
MDPV & Methylenedioxypyrovalerone \\
META & Methadone \\
METH & Methamphetamine \\
METY & Mthylone \\
MOR & Morphine \\
MRM & Multiple reaction monitoring \\
MS & Mass spectrometry \\
MS/MS & Tandem mass spectrometry \\
NADPH & nicotinamide adenine dinucleotide phosphate \\
NAL & Naltrexone \\
\hline M &
\end{tabular}




\begin{tabular}{ll} 
NAPQI & N-acetyl-p-benzoquinone imine \\
NLS & Neutral loss scan \\
NPS & Novel psychoactive substances \\
OTC & Over the counter \\
OXY & Oxycodone \\
PAPS & 3'-phosphoadenosine-5'-phosphosulfate \\
PIS & Precursor ion scan \\
QqQ & Triple quadrupole \\
QTOF & Quadrupole time-of-flight \\
RNS & Reactive nitrogen species \\
ROS & Reactive oxygen species \\
SAM & S-andeosyl-L-methionine \\
TCC & Total compound chromatogram \\
THC & $\Delta^{9}$-tetrahydrocannabinol \\
TIC & Total ion chromatogram \\
TOF & Time-of-flight \\
UHPLC & Ultra high performance liquid chromatography \\
XIC & Extracted ion chromatogram \\
a-PVP & $\alpha$-Pyrrolidinopentiophenone \\
\hline
\end{tabular}




\section{INTRODUCTION}

Xenobiotic drugs have been used by humans for millennia and continue to be a problem today. The current detection methods for drugs of abuse involve the identification of a biomarker of exposure in a bodily matrix; typically blood, urine, or hair. The biomarkers used for drug analysis are either the parent drug or a common metabolite which can be analyzed. For blood and urine analysis, drugs and metabolites are generally only observable for several days, with lipophilic drugs existing in the system for no more than two weeks. For hair analysis, the biomarkers may be detected several months, or possibly years, after ingestion, depending upon the length and rate of growth of the individual's hair. Current hair analysis has many obstacles however, including a lack of consensus regarding incorporation of biomarkers into hair, wide variation of extraction methods, and the limited ability to distinguish between internal drug incorporation and external surface exposure.

A type of biomarker that has not yet been widely applied to the analysis of drugs of abuse involves measurement of covalent protein modifications (i.e., protein "adducts"). When xenobiotics are metabolized, some of the products created may be reactive metabolic species capable of forming covalent bonds with certain amino acid residues in proteins. Such protein adducts are, in general, much longer lived in the body than the parent compound or its metabolites. 
The current research explored the capabilities of a wide variety of drugs of abuse to form covalent protein adducts with cysteine residues present in hemoglobin, a prominent and essential blood protein. This exploration was accomplished by first analyzing the adduct forming capabilities of the drugs with glutathione, a small tripeptide containing a reactive cysteine moiety, in an in vitro metabolic assay system. Following the characterization of the glutathione adducts formed, hemoglobin adducts were created and analyzed using the same in vitro assay, providing insight into the potential covalent adduct forming capabilities of the drugs of interest and the properties and localization of the generated protein modifications. My research establishes the potential for drugprotein adducts to be used as biomarkers of exposure and creates the necessary foundation for future work to continue with these compounds toward the goal of a validated and widely applicable alternative to hair analysis for retrospective abused drug detection.

\section{LITERATURE REVIEW}

\subsection{Drugs of Abuse}

In 2014, there were an estimated 44 million drug users in the United States, with the numbers continually increasing. ${ }^{1}$ Despite their prevalence and popularity in today's society, many plant-based produced drugs of abuse like heroin and cocaine have long and elaborate histories. Opium, from which heroin is derived, can be traced back over 1000 years to the ancient Greeks, whereas 
cocaine was used for religious and ceremonial reasons by the ancient civilizations in the Americas. ${ }^{2,3}$ Over the years, the number of abused drugs has continued to increase and now includes many synthetic compounds in addition to the naturally derived ones. ${ }^{4}$ In the United States, the Drug Enforcement Administration (DEA) categorizes drugs into five schedules according to a drug's medical uses and dependency potential. ${ }^{5}$ Any drug that does not fit into one of the DEA's five schedules is categorized as an over the counter (OTC) medication or non-scheduled prescription medication. Table 1 lists all of the current DEA schedule classifications and the properties of the drugs found in each one.

\begin{tabular}{|c|l|}
\hline Schedule I & $\begin{array}{l}\text { Drugs, substances, or chemicals with no currently accepted } \\
\text { medical use by the federal government and a high potential for } \\
\text { abuse }\end{array}$ \\
\hline Schedule II & $\begin{array}{l}\text { Drugs, substances, or chemicals with high potential for abuse, } \\
\text { but which do have an accepted medical use }\end{array}$ \\
\hline Schedule III & $\begin{array}{l}\text { Drugs, substances, or chemicals with moderate to low potential } \\
\text { for abuse and dependence }\end{array}$ \\
\hline Schedule IV & $\begin{array}{l}\text { Drugs, substances, or chemicals with low potential for abuse } \\
\text { and dependence }\end{array}$ \\
\hline Schedule V & $\begin{array}{l}\text { Drugs, substances, or chemicals with lower potential for abuse } \\
\text { than Schedule IV and preparations containing limited quantities } \\
\text { of certain narcotics }\end{array}$ \\
\hline
\end{tabular}

Table 1. Drug schedules as determined by the U.S. DEA.

Depending upon the source consulted, there are generally anywhere from four to eight recognized classes of abused drugs upon pharmacological effect. ${ }^{6-9}$ These classes include depressants, stimulants, hallucinogens, opioids, antipsychotics, cannabis, and novel psychoactive substances (NPS). ${ }^{10}$ These categories are a result of categorizing the drugs depending primarily upon the 
effect on the central nervous system (CNS) of the user and the source of the compound itself. There will often be some overlap regarding the CNS effects of these classes. For example, opioids have a similar effect to most depressants yet are categorized separately.

The drugs of interest in this study included a-pyrrolidinopentiophenone, acetaminophen, alprazolam, buprenorphine, clozapine, cocaine, diazepam, methadone, methamphetamine, methylenedioxymethamphetamine, methylenedioxypyrovalerone, methylone, morphine, naltrexone, oxycodone, and $\Delta^{9}$-tetrahydrocannabinol. Table 2 shows the schedule and drug class for each of these compounds along with other identifying information. 


\begin{tabular}{|c|c|c|c|c|}
\hline Drug Name & Abbreviation & Formula & Schedule & Class \\
\hline a-Pyrrolidinopentiophenone & $\alpha-P V P$ & $\mathrm{C}_{15} \mathrm{H}_{21} \mathrm{NO}$ & 1 & $\begin{array}{c}\text { NPS/ } \\
\text { Stimulant }\end{array}$ \\
\hline Acetaminophen & APAP & $\mathrm{C}_{8} \mathrm{H}_{9} \mathrm{NO}_{2}$ & OTC & Analgesic \\
\hline Alprazolam & ALP & $\mathrm{C}_{17} \mathrm{H}_{13} \mathrm{CIN}_{4}$ & IV & Depressant \\
\hline Buprenorphine & BUP & $\mathrm{C}_{29} \mathrm{H}_{41} \mathrm{NO}_{4}$ & III & Opioid \\
\hline Clozapine & CLZ & $\mathrm{C}_{18} \mathrm{H}_{19} \mathrm{CIN}_{4}$ & $\mathrm{R}_{\mathrm{x}}$ & Antipsychotic \\
\hline Cocaine & $\mathrm{COC}$ & $\mathrm{C}_{17} \mathrm{H}_{21} \mathrm{NO}_{4}$ & II & Stimulant \\
\hline Diazepam & DZP & $\mathrm{C}_{16} \mathrm{H}_{13} \mathrm{CIN}_{2} \mathrm{O}$ & IV & Depressant \\
\hline Methadone & META & $\mathrm{C}_{21} \mathrm{H}_{27} \mathrm{NO}$ & II & Opioid \\
\hline Methamphetamine & METH & $\mathrm{C}_{10} \mathrm{H}_{15} \mathrm{~N}$ & II & Stimulant \\
\hline Methylenedioxymethamphetamine & MDMA & $\mathrm{C}_{11} \mathrm{H}_{15} \mathrm{NO}_{2}$ & 1 & $\begin{array}{c}\text { Stimulant/ } \\
\text { Hallucinogen }\end{array}$ \\
\hline Methylenedioxypyrovalerone & MDPV & $\mathrm{C}_{16} \mathrm{H}_{21} \mathrm{NO}_{3}$ & 1 & $\begin{array}{c}\text { NPS/ } \\
\text { Stimulant }\end{array}$ \\
\hline Methylone & METY & $\mathrm{C}_{11} \mathrm{H}_{13} \mathrm{NO}_{3}$ & 1 & $\begin{array}{c}\text { NPS/ } \\
\text { Stimulant }\end{array}$ \\
\hline Morphine & MOR & $\mathrm{C}_{17} \mathrm{H}_{19} \mathrm{NO}$ & II & Opioid \\
\hline Naltrexone & NAL & $\mathrm{C}_{20} \mathrm{H}_{23} \mathrm{NO}_{4}$ & $\mathrm{R}_{\mathrm{x}}$ & Opioid \\
\hline Oxycodone & OXY & $\mathrm{C}_{18} \mathrm{H}_{21} \mathrm{NO}_{4}$ & II & Opioid \\
\hline$\Delta^{9}$-Tetrahydrocannabinol & $\mathrm{THC}$ & $\mathrm{C}_{21} \mathrm{H}_{30} \mathrm{O}_{2}$ & 1 & Cannabinoid \\
\hline
\end{tabular}

Table 2. The drugs of interest selected for this study in addition to the abbreviations used for each. Table 2 also lists the formula, US DEA schedule, and general class of each drug of interest.

\subsection{Drug Metabolism}

The lifespan of any xenobiotic within the body can be characterized by its absorption, distribution, metabolism, and excretion (ADME) parameters. Absorption describes the process by which a compound is taken up by the body, and the process is affected by the route of administration. Distribution refers to the overall pharmacokinetics of an absorbed chemical species, specifically the transfer of a chemical from where it was absorbed to where it may have an effect. Metabolism refers to the breakdown of a precursor chemical into its metabolites. These metabolites may be either pharmacologically active or 
inactive. Excretion is the process of removing chemicals and their metabolites from the body; failure to efficiently removed compounds may lead to accumulation over time. As previously stated, the metabolism of a chemical species may result in pharmacologically active metabolites capable of producing unforeseen and toxic results. As a result of factors such as nutritional state, gender, and dosage, no two individuals will metabolize the same drug in the same way ${ }^{11}$, although it is possible to determine general metabolic trends for drugs and classes of drugs.

\subsubsection{Phase I Metabolism}

Metabolic processes may be divided into two categories: Phase I and Phase II metabolism. Phase I metabolic processes generally form metabolites which are more polar than the parent xenobiotic and are typically a means of detoxification (or, in some cases, toxification) and preparation for excretion. Phase I metabolism refers to a number of reactions that a xenobiotic may undergo where a relatively small chemical modification, such as a hydroxylation or an epoxidation, occurs which may slightly increase hydrophilicity. While Phase I metabolic reactions occur in a variety of organs and tissues in the body, these reactions are predominantly carried out by cytochrome P450 (CYP) enzymes in the liver, with some estimates stating this organ accounts for $\sim 90 \%$ of all metabolic activity. ${ }^{12}$

The CYP enzymes are a type of enzyme known as hemoproteins, thus named due to a heme group located in the active site of the protein. For more information regarding the heme complex, see 2.4.4. The heme group is 
responsible for the CYP family's activity as Phase I metabolic enzymes. CYP enzymes are capable of catalyzing otherwise difficult chemical oxidations, including hydroxylation of unfunctionalized alkyl chains ${ }^{13}$, which is accomplished via cycling the oxidation state of the iron within the heme group, as can be seen in Figure 1. This cycling process is proposed many times in the extant literature on CYP enzymes ${ }^{14-16}$. In step (a), the ferric iron of the CYP enzyme is hexacoordinated and in the low-spin state when the substrate to be oxidized $(\mathrm{RH})$ is introduced, leading to the loss of a water molecule and the formation of a highspin penta-coordinated complex (b). Following the reduction of the iron to ferrous $\mathrm{Fe}^{\text {II }}(\mathrm{c})$, the iron can react with an oxygen molecule to form an oxygenated species (d), which is then further reduced to give a peroxy-complex (e). After the addition of two protons, heterolytic cleavage results in an oxo-ferryl oxenoid intermediate (f). A hydrogen atom is then abstracted from the substrate in solution, producing the reduced hydroxy ferric species and substrate radical (g). This production leads to a coordinated oxidized substrate species which then releases the oxidized substrate and produces the initial low-spin ferric compound (a). The CYP enzymes are not only responsible for hydroxylations, as they are capable of performing multiple other oxidative biotranformations on xenobiotics, as seen in Table 3. 


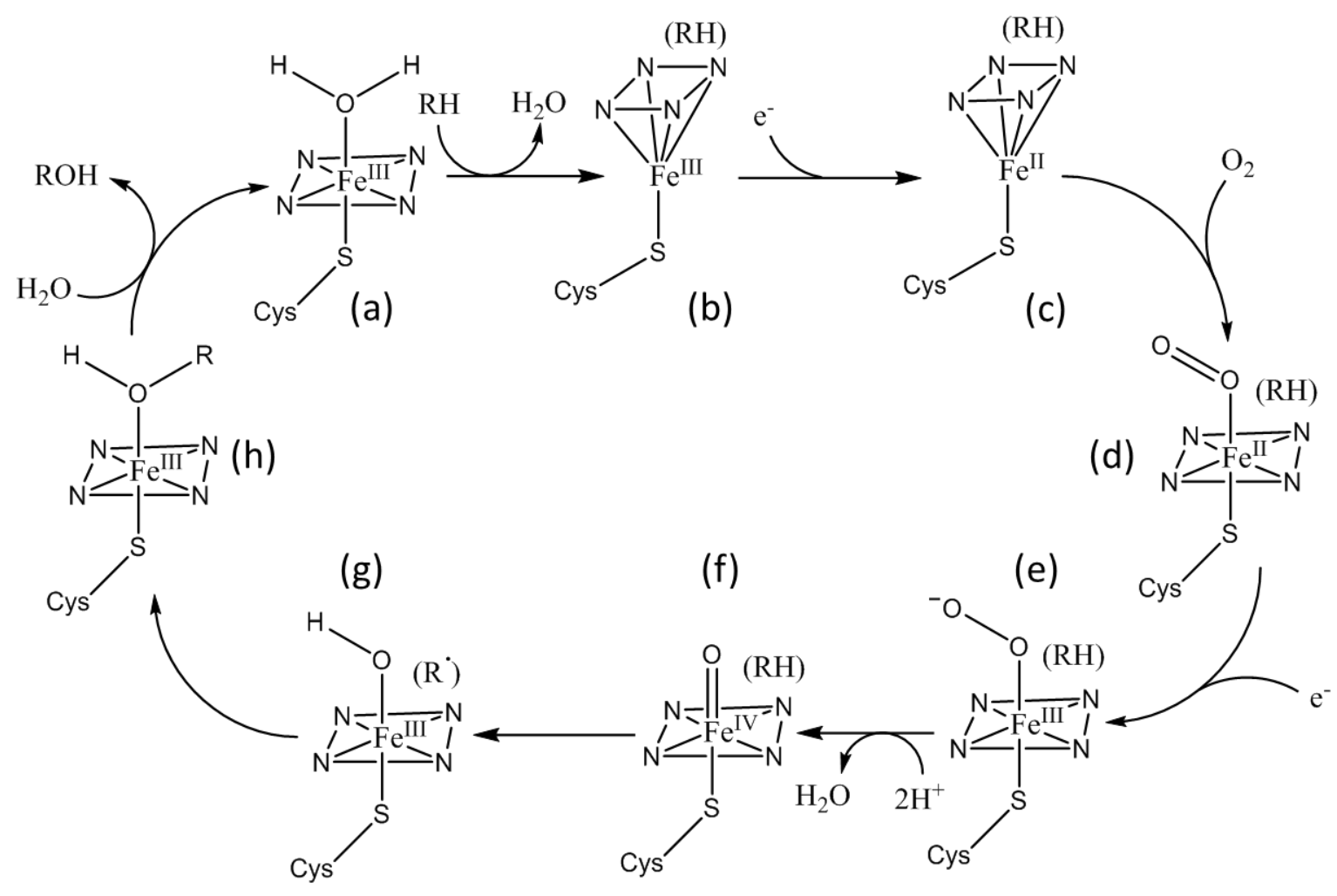

Figure 1. The process of heme cycling by a CYP enzyme to produce an oxidized substrate.

\begin{tabular}{c|c}
$\begin{array}{c}\text { Chemical } \\
\text { group }\end{array}$ & Reactions \\
\hline Alkane & $\begin{array}{c}\text { Hydroxylation, } \\
\text { dehydrogenation } \\
\text { Alkene } \\
\text { Alkyne }\end{array}$ \\
Arene & Oxidation to carboxylic acid \\
Aniline & Hydroxylation, epoxidation \\
Ester & N-hydroxylation \\
Ether & Oxidative cleavage \\
& O-dealkylation
\end{tabular}

Table 3. Some of the chemical groups which may be metabolized by CYP enzymes and the reactions possible with each. 
Another means of oxidation in a molecule is the process of an "NIH" shift, named for the National Institute of Health, where it was first described. ${ }^{17}$ There are several proposed mechanisms by which an NIH shift may occur, however, the prevailing theory, which has been experimentally corroborated, involves formation of an unstable epoxide which then undergoes a hydride shift. ${ }^{17,18}$ While the process is typically followed by a rearomatization step, in situations where GSH or a similar nucleophile is present, rearomatization is not always seen ${ }^{19} \cdot{ }^{19}$

\subsubsection{Phase II Metabolism}

Phase II metabolism refers to reactions where the addition of a large polar moiety substantially increases hydrophilicity, such as conjugation to glucuronic acid. The increase in hydrophilicity results in more facile excretion of the compound from the body in the urine. In contrast, conjugation with glutathione, particularly in the liver, can result in excretion of Phase II metabolites via bile and feces. There are several metabolic processes which may result from Phase II metabolism, as can be seen in Table 4. Table 4 also shows some of the various enzymes and cofactors required for these reactions to take place.

\begin{tabular}{lll} 
Process & Enzyme & Co-Factor \\
\hline Methylation & methyltransferase & $\begin{array}{l}\text { S-andeosyl-L- } \\
\text { methionine (SAM) }\end{array}$ \\
Sulfation & Sulfotransferase & $\begin{array}{l}\text { 3'-phosphoadenosine- } \\
\text { 5'-phosphosulfate } \\
\text { (PAPS) }\end{array}$ \\
Acetylation & N-acetyltransferase & Acetyl coenzyme A \\
$\begin{array}{l}\text { Glucuronidation } \\
\begin{array}{l}\text { Glutathione } \\
\text { conjugation }\end{array}\end{array}$ & UDP-glucuronosyltransferase & UDP-glucuronic acid \\
\hline
\end{tabular}

Table 4. Phase II metabolic processes and the respective enzymes and co-factors required for each 


\subsection{Biomarkers of Exposure}

Biomarkers of exposure are entities which are indicative of exposure to a chemical or class of chemicals. In terms of drugs of abuse, the most commonly analyzed biomarkers to determine exposure are either the parent drug or a stable metabolite. Since metabolism primarily occurs in the liver and metabolic processes are designed to facilitate excretion, biomarkers are selected on the basis of the specific matrix collected for analysis. Although there are many different matrices that may be utilized for the analysis of drugs, the three primary

ones are blood, urine, and hair, with each offering benefits and drawbacks. ${ }^{20-22}$

\subsubsection{Blood and Urine as a Matrix}

Blood and urine are both well-characterized matrices with extensive protocols for exposure biomarker analysis established in the literature. ${ }^{22-25}$ While it may be possible to detect precursor drugs and their metabolites in both blood and urine, precursor drugs are typically used as biomarkers in blood and metabolites used as biomarkers in urine. Since blood and urine are both aqueous matrices, the utilization of liquid chromatographic (LC) separation in analysis requires fewer steps, as there are typically few solubility issues with the desired biomarker analytes. While a plethora of information already in the literature addresses many potential problems with the analysis of drugs in both blood and urine, they each still have drawbacks. Both blood and urine are collected via invasive means, leading to privacy issues in collection. ${ }^{26}$ Blood and urine also have limited windows of detection for currently utilized biomarkers. ${ }^{27}$ Depending upon the properties of the drug, the window of detection may be less than a 
day $^{28}$, with some more lipophilic drug biomarkers being detectable for no more than about a few weeks. ${ }^{29} \mathrm{~A}$ comparison of several drugs of abuse and their relative windows of detection in both blood and urine can be found in Table $5 .^{28}$

\begin{tabular}{cccc} 
Parent Drug & Analyte & $\begin{array}{c}\text { Detection Time in } \\
\text { Blood }(\mathrm{h})\end{array}$ & $\begin{array}{c}\text { Detection } \\
\text { Time in Urine } \\
(\mathrm{h})\end{array}$ \\
\hline Methamphetamine & Methamphetamine & 48 & $87 \pm 51$ \\
MDMA & MDMA & 24 & 48 \\
Cannabis & THC & 5 & N/A \\
Cannabis & THCCOOH & 36 & 87 \\
Heroin & Morphine & 20 & $11-54$
\end{tabular}

Table 5. A summary of the retention times in blood and urine for methamphetamine, MDMA, cannabis, and heroin, as reported in hours.

\subsubsection{Hair as a Matrix}

The use of hair as a matrix is another common approach to drug analysis and exposure assessment. The literature regarding hair analysis is not as encompassing as it is for either blood or urine, but there are still many protocols published for the analysis of drugs in hair. Hair is collected through less invasive means than both blood and urine, and the window of detection for hair analysis can be significantly longer, with the potential for drugs to persist in hair for months or years. ${ }^{21} \mathrm{~A}$ comparison of detection windows for each matrix can be found in Figure 2. In addition to the extended window of detection, the use of hair as a matrix also allows for segmental analysis. Segmental analysis consists of the partitioning of the hairs collected and analyzing each partition separately, allowing for a timeline of previous drug exposure to be compiled for an individual. 
${ }^{30}$ The biomarkers typically examined in hair analysis are both parent drugs and their metabolites. ${ }^{31}$ Unfortunately, there is no single incorporation mechanism for drugs into the hair, as direct incorporation into hair from blood, adsorption from sweat, and adsorption from smoke or powders are all potential routes of incorporation. ${ }^{32}$ Additionally, in the existing protocols, there is no way to distinguish biomarkers present from ingestion of a compound from biomarkers present from surface contamination of the hair without first utilizing extensive washing procedures to remove external contaminants. ${ }^{33}$ These washing procedures are not guaranteed to remove all of the external contaminants, as some drugs may bind more strongly to hair than others. ${ }^{34}$ In addition, washing procedures may also result in loss of drug that was incorporated from the blood. Lastly, since hair is a solid matrix, there are several steps required prior to analysis to extract and clean up the sample to analyzed. ${ }^{30}$

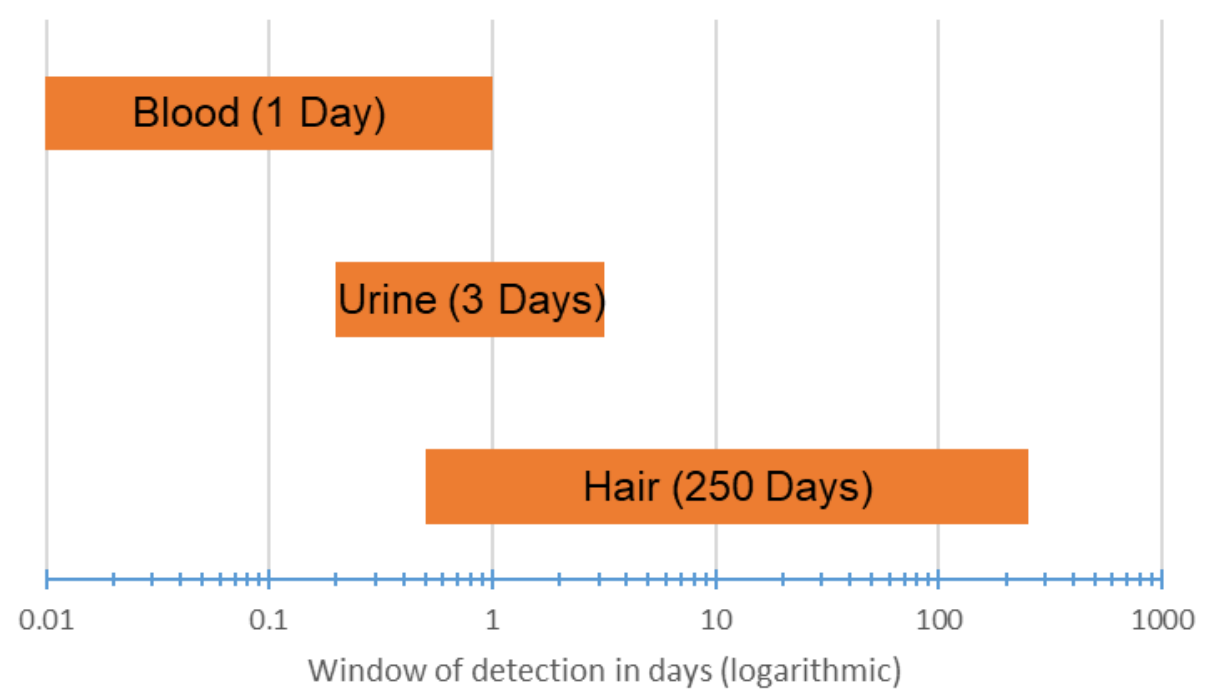

Figure 2. The window of detection for cocaine and its metabolites in blood, urine, and hair set to a logarithmic scale. ${ }^{35}$ 


\subsection{Use of Protein Adducts as Exposure Biomarkers}

The drawbacks in the use of blood, urine, or hair as testing matrices for drugs and other xenobiotics has led to interest in the use of alternative matrices or biomarkers to bypass these difficulties. One such example is the use of protein adducts as biomarkers of exposure. Protein adducts are formed as the result of a covalent modification to a protein by a directly reactive xenobiotic or a metabolite formed by the xenobiotic. Covalent protein adduction is common occurrence in the body, with many modifications being of relatively low molecular weight, for example reactive oxygen species (ROS) $)^{36,37}$ and reactive nitrogen species (RNS) ${ }^{38,39}$. Larger modifications are possible however, with examples such as glutathionylation and lipid peroxidation product adduction. ${ }^{40}$ While some protein modifications may be unstable in certain protein microenvironments, as is the case with compounds such as 4 -hydroxy-2-nonenal ${ }^{41}$, in general, covalent protein adduct formation is an irreversible phenomenon ${ }^{42}$. Unlike DNA, essentially no cellular repair mechanisms exist for covalent protein modifications ${ }^{42}$, meaning that most protein adducts are typically extant for the remaining life of the protein.

Recognition of the adduction of reactive electrophiles created in vivo to proteins first emerged in the 1930s and 1940s, first with Fieser's work regarding the hepatotoxicity of various hydrocarbons ${ }^{43}$ and then with work done by Miller and Miller regarding the binding of aminoazo dyes to the cellular constituents of the livers of rats fed $p$-dimethylaminoazobenzene, a known hepatic carcinogen ${ }^{44}$. These studies laid the groundwork for what became known as the "covalent 
binding theory", which proposed that certain chemicals were able to covalently bind to macromolecules in the body causing toxicity. ${ }^{45}$ This progression continued when Brodie, et al. were able to show that bromobenzene is metabolically activated and covalently binds to proteins in the liver, resulting in liver necrosis. ${ }^{46}$ Since then, the concept of xenobiotic induced protein adduction has continued to expand and is now a major area of interest for new drug development where such adducts may mediate drug toxicity or participate in the therapeutic mechanism of certain drugs. ${ }^{45}$

The use of protein adducts as exposure biomarkers is also not a new concept. Since the idea of utilizing them for the biomonitoring of various compounds was first suggested in the 1970 s $^{46,47}$, research regarding protein adducts has continued to expand. In 1986, Skipper et al. looked at new analytical methods to measure protein adducts with 4-aminobipheny ${ }^{48}$, an arylamine capable of causing bladder cancer in human and dogs. ${ }^{49}$ Research published in 1989 by Tornqvist et al. analyzed protein adducts formed upon exposure to four types of gasoline and diesel exhaust. ${ }^{50}$ In 1993, Christakopoulos et al. characterized styrene-protein adducts present in authentic collected blood samples from factory workers exposed to styrene. ${ }^{51}$

\subsubsection{Protein Adduction by Drugs of Abuse}

With few exceptions, there are only limited data available regarding the potential for important drugs of abuse to form covalent adducts with blood or tissue proteins. One of the major exceptions is ethanol, where its ability to interact with proteins has been widely studied. Ethanol's major metabolite, 
acetaldehyde, is known to form adducts with proteins. The reaction between acetaldehyde and serum albumin was first characterized in $1982^{52}$, and the reaction with hemoglobin was first characterized in $1985^{53}$. Acetaldehyde's protein interactions are generally the result of the formation of an unstable Schiff base, which then is able to interact with nucleophilic amino acids of proteins. ${ }^{54}$ These bases may form stable or unstable adducts, and the stable adducts may be useful as a means of retrospective detection of exposure..$^{55,56}$

Some data are also available for protein adduction by the licit drug morphine, which is both itself abused and that is a major metabolite of the illicit drug heroin. A major reactive metabolite of morphine, morphinone, has been shown, in both in vitro and in vivo studies, to covalently bind to free thiols of proteins and peptides found in the body. ${ }^{56-59}$ In vitro studies by Correia, et al. suggested that formation of a more reactive and unstable reactive species is the preferred biological transformation of morphine, as the glutathione-morphine adduct formed by this reactive intermediate was observed at levels two-fold greater than those of normorphine.${ }^{60}$ Previous work Schneider and DeCaprio also demonstrated the ability of morphine metabolites to form adducts with $\mathrm{N}$ acetylcysteine, glutathione, and a synthetic hexapeptide. ${ }^{56}$

Another example of an illicit drug which has shown the capability of binding to protein is cocaine. ${ }^{61}$ The major site of metabolic activity for cocaine is reported to be the tropane nitrogen, with numerous oxidative products capable of being formed at this site. ${ }^{62}$ Cocaine adducts formed with liver proteins have been observed in several studies, with further studies into potential hepatotoxic stress 
caused by these adducts. ${ }^{63,64}$ In 2002, Deng, et al. reported high levels of covalent binding between metabolically activated cocaine and blood proteins. ${ }^{65}$ Additional work by Schneider and DeCaprio investigated the binding of cocaine to glutathione, a synthetic hexapeptide, and $\mathrm{N}$-acetylated variants of the amino acids lysine (Lys), cysteine (Cys), and histidine (His). ${ }^{61}$ Schneider and DeCaprio also posited that epoxidation of the phenyl ring of cocaine is the first step in the formation of cocaine adducts, suggesting that the phenyl ring is the site of adduction. ${ }^{61}$ This reaction scheme contrasts with what was reported in the 1980 s and 1990s that the tropane nitrogen was the likely site of adduction. ${ }^{66,67}$

\subsubsection{Hard-Soft Acid-Base Theory}

The formation of covalent adducts from the reaction of electrophilic xenobiotics with cellular nucleophiles, including proteins, can be generally modeled by the "Hard and Soft Acids and Bases" (HSAB) Theory. The HSAB Theory states that nucleophiles and electrophiles can be categorized on a spectrum from hard to soft, depending on polarizability. Polarizability is a characteristic describing electron density for atoms or molecules, and it applies to the valence electrons in an atomic or molecular electron cloud. ${ }^{68}$ If the valence electrons in the cloud are occupying a large volume of space, they will be more susceptible to outside influence and thus be more polarizable than valence electrons which are closer to the nucleus and relatively less mobile. These electrons are considered more polarizing. ${ }^{69}$ Using this nomenclature, "hard" refers to a nucleophile or electrophile that is relatively small and highly polarizing. Alternatively, "soft" refers to a nucleophile or electrophile that is relatively large 
and highly polarizable. According to HSAB Theory, hard electrophiles will tend to react with hard nucleophiles and vice versa for soft electrophiles and nucleophiles.

A species' hardness or softness cannot be directly measured, but instead can be calculated via its relation to other properties of the compound. The energy levels of the outermost or frontier molecular orbitals (FMOs) are the most important component in determining a compounds "hardness". ${ }^{61} \mathrm{~A}$ species' hardness $(\eta)$ is equal to the energy of the Lowest Unoccupied Molecular Orbital (LUMO) minus the energy of the Highest Occupied Molecular Orbital (HOMO) divided by 2 (Equation 1). A species' softness $(\sigma)$ is then defined as the inverse of the hardness (Equation 2).

$$
\begin{aligned}
& \text { (1) } \eta=\frac{E_{L U M O}-E_{\text {HOMO }}}{2} \\
& \text { (2) } \sigma=\frac{1}{\eta}
\end{aligned}
$$

When dealing with electrophilic species, it is generally the case that a higher softness results in more facile formation of an adduct with a soft nucleophile. ${ }^{68}$ The electrophilic index $(\omega)$ is a parameter that combines softness and electric potential $(\mu)$, or the propensity for a compound to undergo a chemical change (Equations 3 and 4).

$$
\begin{aligned}
& \text { (3) } \mu=\frac{E_{L U M O}+E_{\text {HOMO }}}{2} \\
& \text { (4) } \omega=\frac{\mu^{2}}{2 \eta}
\end{aligned}
$$

The equations presented thus far only apply to an individual species and do not provide insight into bond forming capabilities between two compounds. For this, 
the nucleophilic index $\left(\omega^{-}\right)$can be calculated to predict the likelihood that an adduct will form between nucleophile A and electrophile B (Equation 5). ${ }^{70}$

$$
\text { (5) } \quad \omega^{-}=\frac{\eta_{A}\left(\mu_{A}-\mu_{B}\right)^{2}}{2\left(\eta_{A}+\eta_{B}\right)^{2}}
$$

When dealing with biological species, heteroatoms such as sulfur, oxygen, and nitrogen are the most prominent nucleophilic sites. ${ }^{71}$ Specifically when dealing with proteins, the sulfurs from amino acid thiol residues react as relatively soft nucleophiles. ${ }^{72}$ The softness of the thiol moiety increases as it is converted to a thiolate $\left(\mathrm{SH} \rightarrow \mathrm{S}^{-}\right)$ion, and the thiolate ion is one of the most reactive biological nucleophiles. ${ }^{68}$ The base $\mathrm{pK}_{\mathrm{a}}$ of cysteine is $\sim 8.3$, meaning that at physiological $\mathrm{pH}(\sim 7.4)$ most of the cysteine residues present should be unionized and not in the more reactive thiolate form ${ }^{73}$ However, the formation of the thiolate ion by cysteine residues may be promoted by the three-dimensional interactions between cysteine and any surrounding basic amino acid residues. ${ }^{74}$ Because of the softness of these thiol residues, cysteines have become a major site of interest in regards to formed protein adduct investigations.

Two key components of new drug discovery and development are HSAB interactions and the potential for drug-protein adduct formation. ${ }^{75}$ Concern for lack of protein-binding specificity and potential for unknown side effects has led pharmaceutical companies to avoid drugs that show the capability for covalent protein binding.$^{76}$ Despite this, Robertson found in 2005 that 25 of 71 biological targets examined were covalently bound by marketed drugs. ${ }^{77}$ Since so many compounds are capable of these drug-protein interactions, some researchers have begun exploring what are termed covalent inhibitors, or drugs which 
covalently bind to a protein causing inhibition. ${ }^{78}$ Whether protein adduction is the desired outcome or a negative side effect, HSAB theory is key in the preliminary steps of drugs development and discovery.

\subsubsection{Glutathione}

One prominently reactive biological thiol can be found as part of glutathione (GSH), an endogenous tripeptide which plays an important role in the removal of reactive oxygen species (ROSs) in the body. The structure of GSH consists of glutamic acid, cysteine, and glycine, with a y-peptide linkage between glutamic acid and cysteine (See Figure 3). Endogenous concentrations of GSH in human cells range up to $10 \mathrm{mM}$, with higher concentrations occurring in the liver. ${ }^{79}$ The free thiol moiety of GSH acts as a reactive nucleophilic site, and it has been shown to covalently bind to electrophiles in vivo. ${ }^{80,81}$ The capability of GSH to bind to reactive metabolites allows it to function as one of the primary cellular defenses against electrophilic/oxidative stress and damage. Because of GSH's reactivity and prevalence in the body, GSH adducts have also been used as markers to identify, analyze, and monitor exposure to and excretion of compounds of interest. ${ }^{58,82-84}$ The chemistry and structure of GSH make it an ideal candidate for a trapping agent when utilized in an in vitro metabolic assay. ${ }^{85-87}$ 


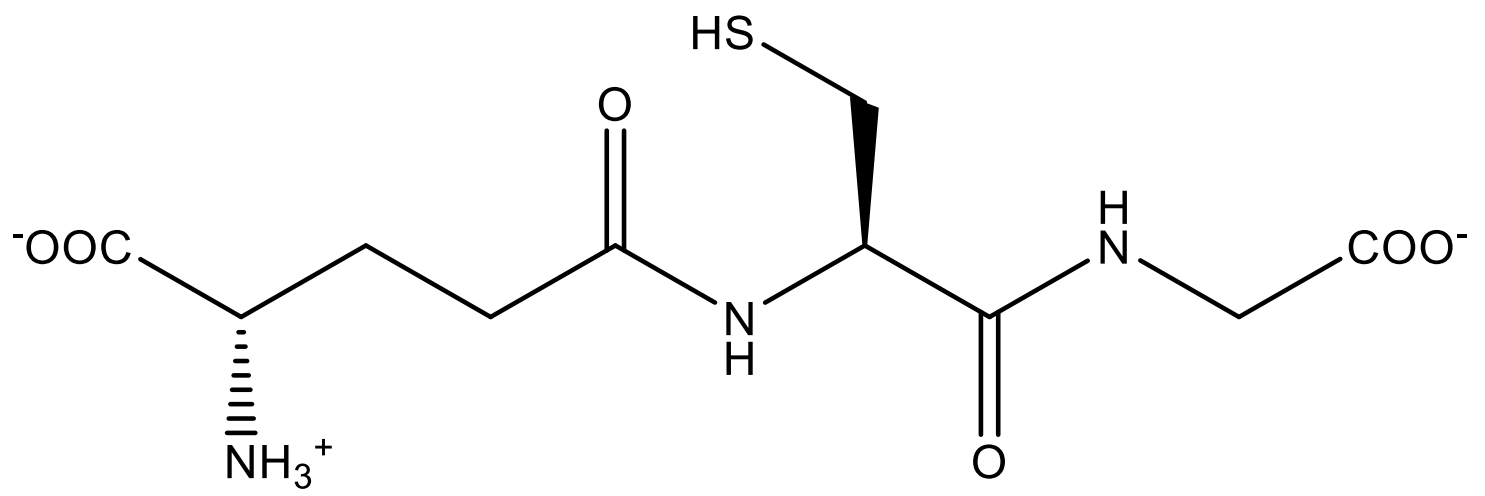

Figure 3. The chemical structure of glutathione showing the appropriate charges on the terminal carboxy groups and the amine group.

Modification of GSH by both licit and illicit drugs has been well-studied and reported in the literature..$^{56,61,84,88-92}$ The first recorded observance of a GSH adduct with acetaminophen was reported by Dahlin et al. in 1984. They reported that $N$-acetyl-p-benzoquinone imine (NAPQI), a reactive metabolite of acetaminophen, was reduced and formed a covalent conjugate with the GSH, although they could not confirm the presence of NAPQI in the assay. ${ }^{80}$ Work in 1988 by the National Institute of Environmental Health Sciences explored the HPLC-MS analysis of GSH conjugates formed with compounds such as acetaminophen and 3-methylindole. ${ }^{93}$ Fragmentation characterization of a GSH adduct formed with a derivative of acetaminophen was performed in 1993 by Ballie and Davis. ${ }^{94}$ Furthermore, a wide variety of licit drugs, such as troglitazone, dantrolene and raloxifene ${ }^{90}$; nefazodone ${ }^{92}$; and clozapine, naproxen, and ibuprofen ${ }^{88}$, have all been analyzed as GSH adducts.

Although not as widely studied as licit compounds or endogenous ROSs, GSH adducts with common illicit drugs of abuse have been reported throughout the literature. One predominant example is 3,4- 
methylenediaminemethamphetamine (MDMA). Ramaley et al., who explored the in vitro metabolism of MDMA with GSH present in the assay, observed two possible GSH adducts; one formed with MDMA and the other with the oxidized catechol of MDMA, 3,4-dihydroxymethamphetamine. ${ }^{95}$ Meyer et al. further explored the adduct forming capabilities of 15 methylenedioxy designer drugs, including both MDMA and methylenediaminepyrovalerone (MDPV), and characterized the major fragments for the observed adducts. ${ }^{84}$ They were able to successfully identify common fragmentation patterns which applied to methylenedioxy designer drugs and their major metabolites. As previously mentioned, work performed by Schneider and DeCaprio characterized the covalent conjugates formed by GSH and the drugs morphine, cocaine, and methamphetamine, and delved into the kinetics and electronics involved in the formation of the observed adducts. ${ }^{56}$ Morphine is another commonly abused drug which has a GSH adduct which has been observed. In 2005, Todaka et al. reported a GSH adduct with morphinone, the major metabolite of morphine, for the first time..$^{58}$

\subsubsection{Hemoglobin as a Target for Reactive Xenobiotics}

Hemoglobin $(\mathrm{Hb})$ is a protein found in high concentrations in erythrocytes (red blood cells). The protein is used for the transport of oxygen and carbon dioxide throughout the body. In adults, erythrocytes are produced in the bone marrow through a process called erythropoiesis. Erythrocytes have a biconcave symmetrical structure and contain no nucleus. Hemoglobin derives its name from the two heme groups contained within its four globin protein subunits. Heme is an 
iron coordination complex, where the central iron atom is coordinated to the four nitrogen atoms of a porphyrin ring system. The specific heme found in hemoglobin is Heme B (Figure 4).

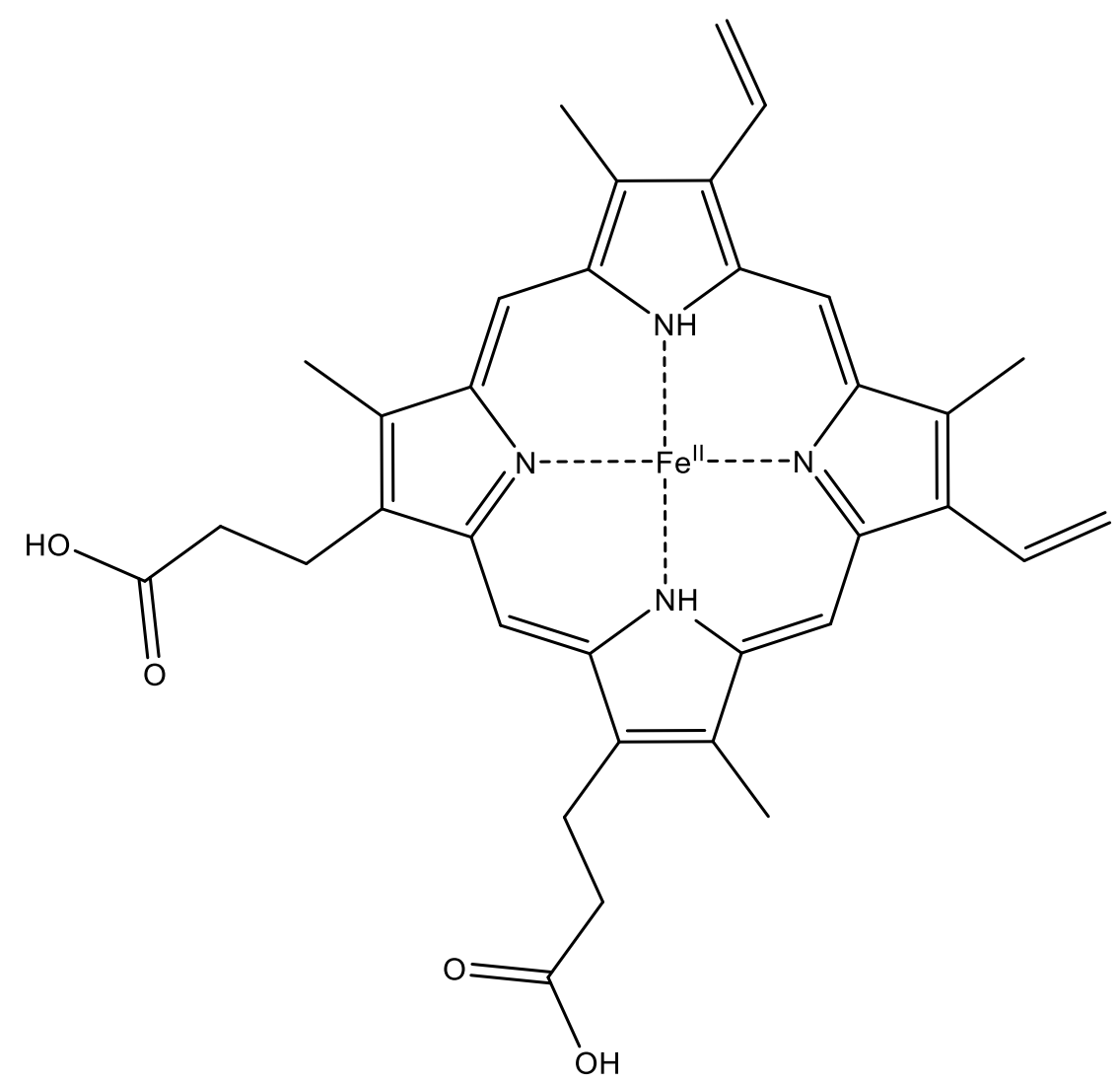

Figure 4. The porphyrin ring system found in the heme B iron complex of hemoglobin.

There are six potential subunits of the $\mathrm{Hb}$ protein, however at least $95 \%$ of the $\mathrm{Hb}$ found in an adult human is made up of the two subunits alpha and beta. ${ }^{96}$ The amino acid sequences of the alpha subunit $(\mathrm{Hba})$ of $\mathrm{Hb}$ was first reported in $1962^{97}$, with the beta subunit $(\mathrm{Hb} \beta)$ sequence being published just one year later in $1963^{98}$. There are only three cysteine residues contained in $\mathrm{Hb}, \mathrm{Hb} \mathrm{a}^{104} \mathrm{Cys}$, $\mathrm{Hb} \beta^{93} \mathrm{Cys}$, and $\mathrm{Hb} \beta^{112} \mathrm{Cys}$. Of these residues, $\mathrm{Hb} \beta^{93} \mathrm{Cys}$ has been shown to be the most susceptible to adduction by soft electrophiles, followed by $\mathrm{Hb} \beta^{112} \mathrm{Cys}$ 
and $\mathrm{Hb} \mathrm{a}^{104} \mathrm{Cys}$, respectively. ${ }^{99}$ The lifespan of erythrocytes in human blood, and thus of $\mathrm{Hb}$, is approximately 120 days, implying that the maximum lifespan for an adduct at any of these three cysteines would be 120 days. ${ }^{100}$

A key issue when utilizing hemoglobin adducts as exposure biomarkers is the capability of xenobiotics to enter into the erythrocyte to interact with the hemoglobin molecule. Erythrocytes generally travel through body about once every minute, totaling about $250 \mathrm{~km}$ throughout the lifespan of a single cell ${ }^{101}$, meaning that there is ample opportunity for xenobiotics and their metabolites to interact with the cells. While hemoglobin is contained within the cellular membrane of the erythrocyte, studies have shown that many compounds are capable of passing through this membrane to potentially reach the protein. ${ }^{101}$ There are several examples in the literature regarding uptake of chemicals by erythrocytes, including cyclosporine $\mathrm{A}^{102}$, the carbonic anhydrase inhibitor MK$927^{103}$, and chloroquine ${ }^{104}$. Although other factors, such as temperature and $\mathrm{pH}$, have been shown to play a role in the uptake of certain compounds by erythrocytes ${ }^{105}$, the above mentioned species are all partitioned via passive diffusion through the cellular membrane. ${ }^{101}$

Hemoglobin adducts have been utilized as biomarkers of exposure to xenobiotics in the past. The compounds which have been studied up to this point have primarily been focused on environmental exposure to toxic chemicals. In vivo experiments in the early 1990s used rats exposed to over 40 arylamines, including several pesticides, to analyze the adducts formed with hemoglobin. ${ }^{106,107}$ These studies noted the potential for the use of hemoglobin 
adducts as a biomarker of exposure to toxic pesticides. The focus on arylamine toxicity and adduct formation continued in 1996, when McClure et al. described hemoglobin adducts formed with the herbicides propanil and fluometuron and proposed a mechanism of adduction for these compounds. ${ }^{108}$ Another application of hemoglobin adduct biomarkers is the exposure to chemical warfare agents, in particular sulfur and nitrogen mustard gases. Sulfur and nitrogen mustards have both been shown to bind covalently to hemoglobin at multiple nucleophilic residues, including cysteine and terminal valine residues and to a lesser extent histidine and lysine residues. ${ }^{73,109}$

\subsection{LC-MS Analysis of Covalent Protein Modifications}

Since peptides and proteins are non-volatile compounds and extracted solutions of such compounds from biological matrices will contain hundreds to thousands of components with varying masses, the mass spectrometric analysis of such species generally requires LC separation prior to analysis. The LC separation requires a liquid solution containing the analytes be passed through a column packed with a sorbent material. As the solution passes through the column, the analytes interact with the sorbent material, and the strength of these interactions affects how quickly the analytes pass through the column. Thus, LC is a means to separate compounds via overall retention times within the column. Following LC separation, mass spectrometry (MS) is used to analyze the compounds as they are eluted from the column. Mass spectrometry is the 
analysis of chemicals by a mass-to-charge ratio $(\mathrm{m} / \mathrm{z})$. Using the $\mathrm{m} / \mathrm{z}$, the projected mass of a chemical species can be predicted.

Mass spectrometers can also provide information about the overall structure of a compound using fragmentation produced by collision induced dissociation (CID). The fragmentation resulting from CID is caused by high speed collisions occurring between a neutral gas molecule and a chemical analyte in the gaseous phase. When the molecules collide, kinetic energy from the neutral gas is transferred to the analyte resulting in bond breakage. Fragmentation patterns may be specific to certain functional groups or structural components. The process of data collection for the precursor analyte and the fragments produced by CID is referred to as tandem mass spectrometry, or MS/MS analysis.

Two common mass analyzers for MS/MS are the triple quadrupole ( $Q q Q)$ and the quadrupole time-of-flight (QTOF). A QqQ MS, as the name suggests, consists of three quadrupoles in series. The first of these quadrupoles (Q1), is used to select which ions are allowed to pass through the instrument. The second quadrupole (q or Q2), is used as a collision cell for CID to produce characteristic fragments. The third quadrupole (Q3), is used to select which specific fragments produced in the collision cell will pass through to the detector. There are several scanning modes possible with a QqQ depending upon which $\mathrm{m} / \mathrm{z}$ ratios are selected, including as product ion scans, precursor ion scans (PIS), neutral loss scans (NLS), and selected reaction monitoring or multiple reaction monitoring (MRM) (Figure 5). 


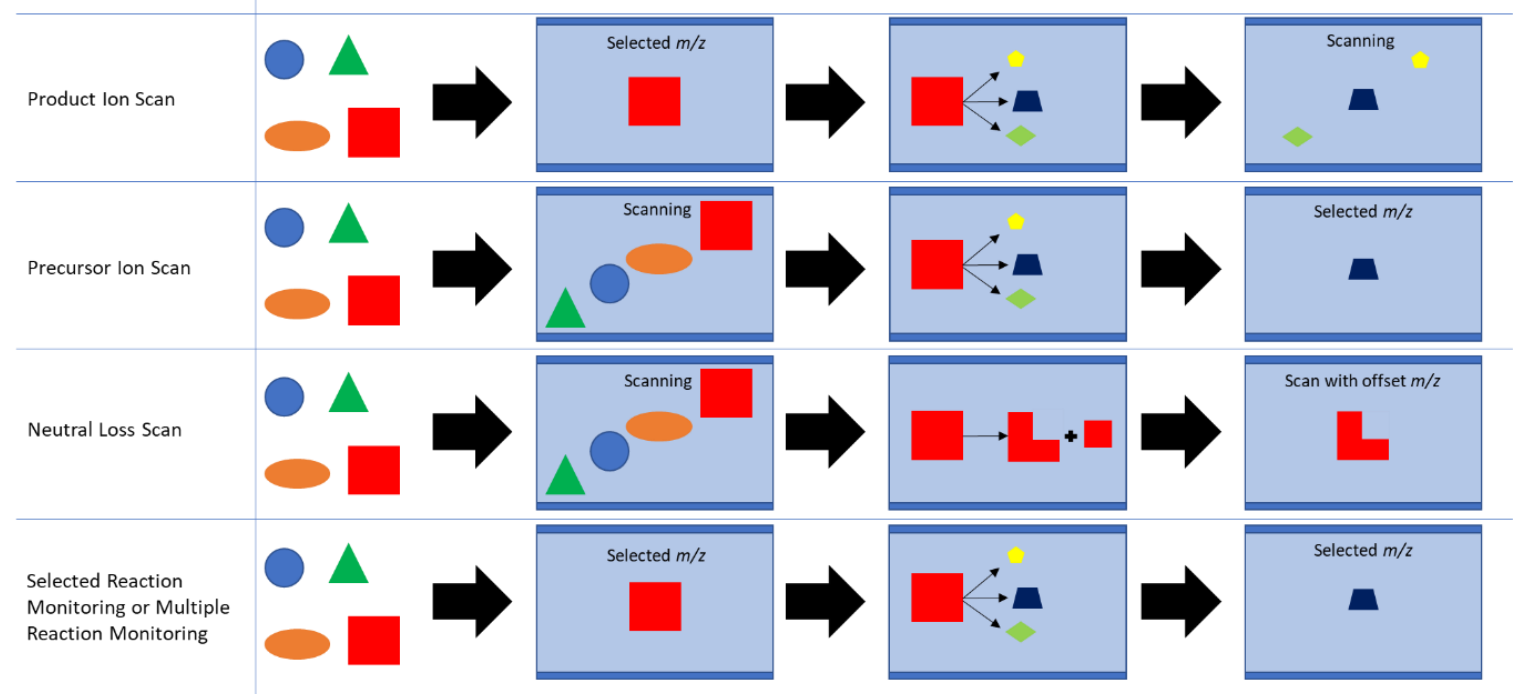

Figure 5. This diagram shows the four major scanning modes for an LC-QqQ-MS system.

Of particular interest in the present research is the PIS, as certain peptides such as GSH have specific fragmentation patterns which are indicative of GSH adducts. ${ }^{110}$ Negative mode analysis of GSH-containing compounds provides very characteristic fragments at $m / z 306,272,254,210,179,160,143$, and 128 , with more fragments possible depending upon the ionized molecule mass, or $[\mathrm{M}+\mathrm{H}]^{+}$, of the compound. ${ }^{88,110}$ For example, $[\mathrm{M}+\mathrm{H}]^{+}-273$ refers to an indicative fragment where the precursor adduct is missing a portion with a $\mathrm{m} / \mathrm{z}$ of 273. These specific fragmentation patterns of some peptides, in particular GSH, allows for more facile examination of these species than when utilizing positive ionization.

The QTOF mass analyzer consists of an initial quadrupole for ion selection in tandem with a time-of-flight (TOF) chamber. All ions that pass 
through the collision cell enter the chamber are given the same kinetic energy in a single direction by the orthogonal accelerator. The ions then all interact with the reflectron, which forces the ions back to the detector (Figure 6). This particular means of compound separation allows for high mass resolution and mass accuracy, which is helpful when analyzing complex peptide mixtures and is necessary for protein analysis. ${ }^{111,112}$

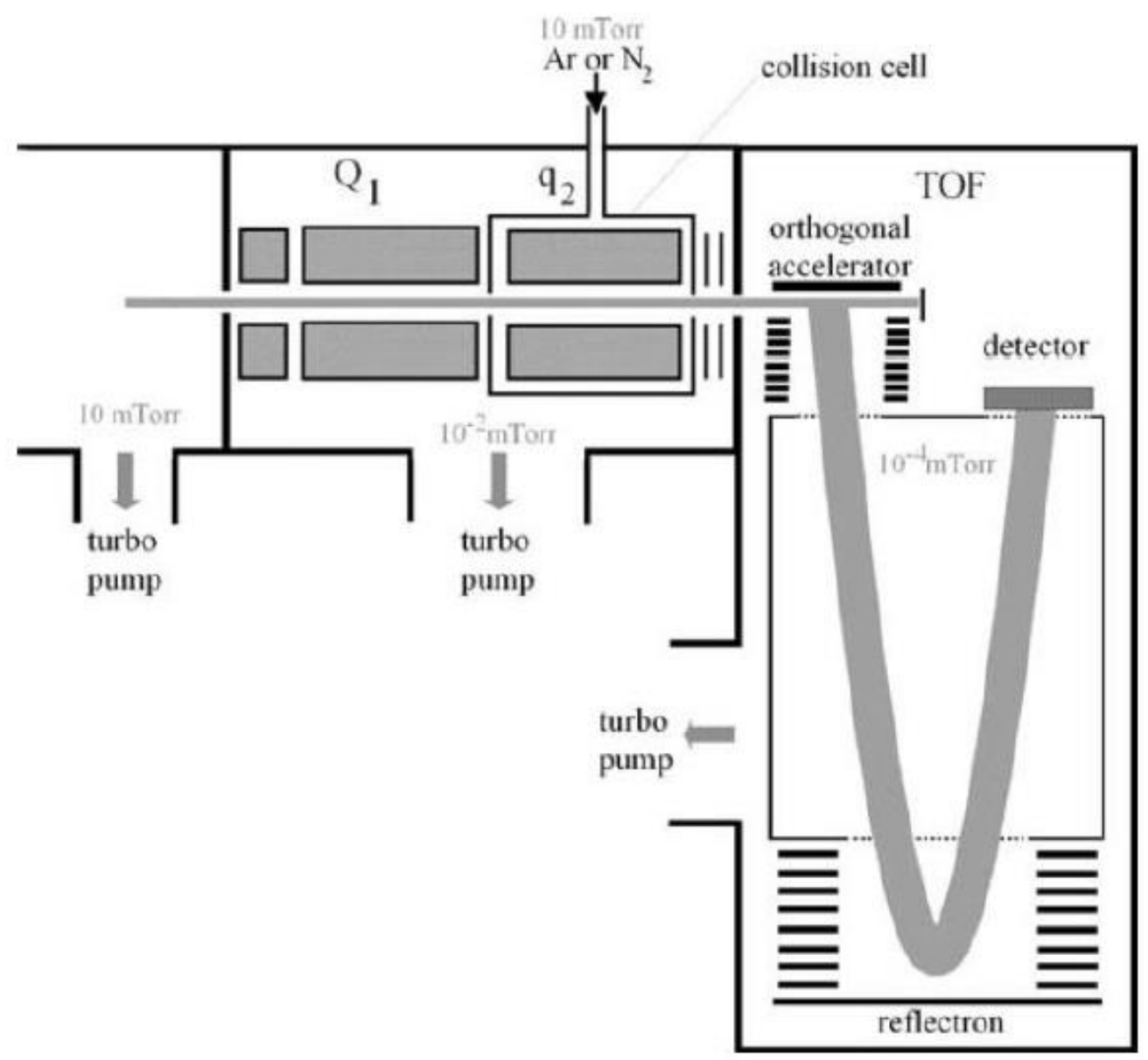

Figure 6. Schematic for a generic QTOF instrument, showing the quadrupole for ion selection $\left(Q_{1}\right)$, the collision cell $\left(q_{2}\right)$, orthogonal accelerator, reflectron, and detector. ${ }^{112}$ The gas used in the collision cell for this research was $N_{2}$. 


\subsection{Bottom-Up Proteomics}

The MS analysis of proteins such as $\mathrm{Hb}$ is generally divided into three categories: top-down proteomics, middle-down proteomics, and bottom-up proteomics (Figure 7). Top-down proteomics refers the analysis of proteins by introducing a whole protein into the mass spectrometer and then fragmenting the protein into peptides via CID. The peptides are then analyzed to determine the overall structure and sequence, thus identifying the protein and its modifications. Middle-down proteomics utilizes a proteolytic digestion to break down the protein into mid-range molecular mass peptides before introduction into the mass spectrometer. The use of tandem mass spectrometry (MS/MS) allows for fragmentation of the enzymatically-produced peptides, and these data can provide insight into the amino acid chains which makes up the peptides. Bottomup proteomics is very similar to middle-down proteomics, except that it uses a proteolytic enzyme to break the protein into much smaller peptide chains. These are more useful than the mid-range peptides when the protein being analyzed is well-characterized and the sequence is already known. 


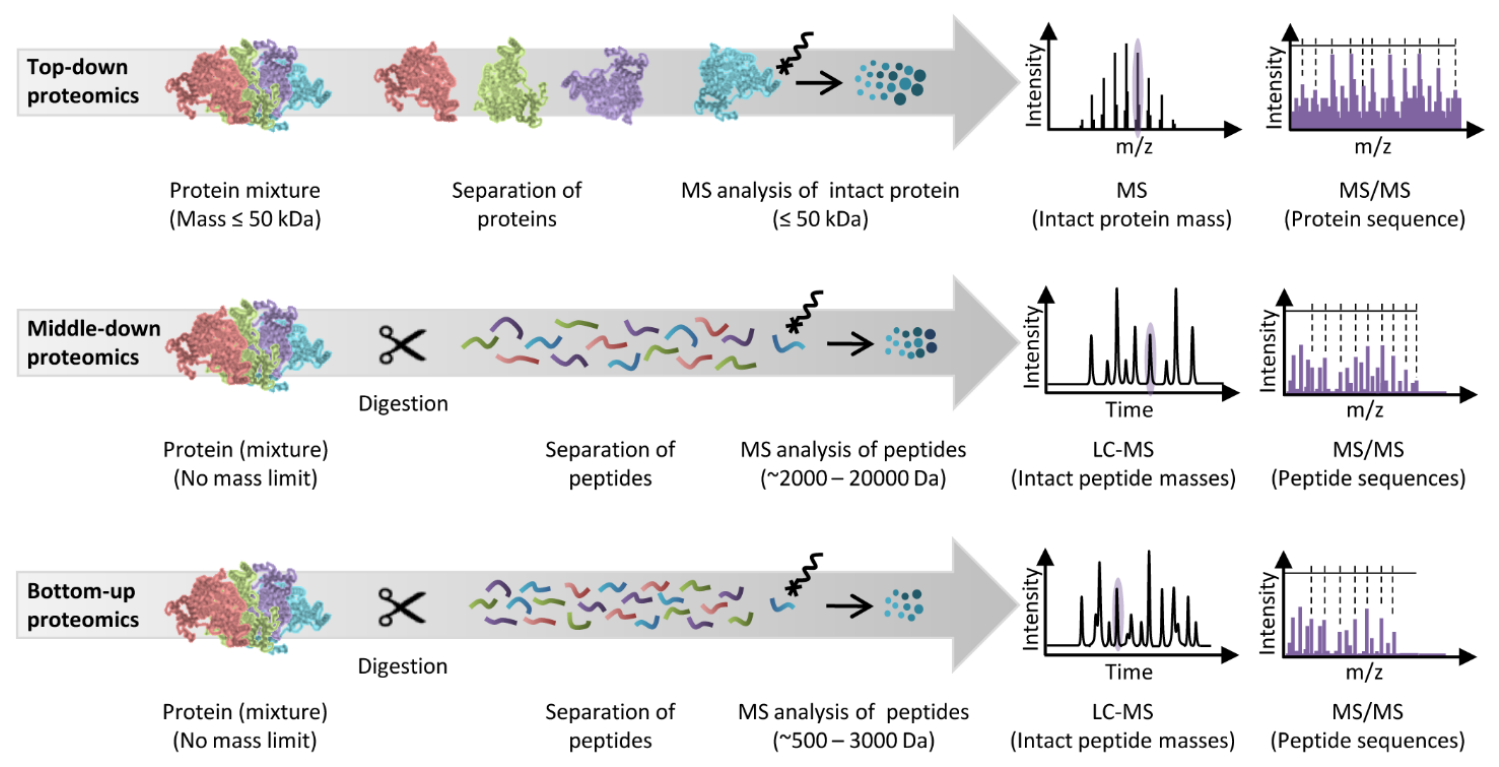

Figure 7. This diagram shows the general procedures for top-down, middle-down, and bottom-up proteomics. ${ }^{113}$

When a proteolytic enzyme with a well-characterized cleavage pattern, such as trypsin, is used for the protein digestion prior to analysis in bottom-up proteomics, the resulting peptides can be analyzed with a high degree of accuracy. Trypsin is an enzyme that cleaves peptides at the carboxylic end of both lysine and arginine residues, except when followed by proline, with very high specificity. ${ }^{114}$ This specificity implies that a protein with a known amino acid sequence, such as $\mathrm{Hb}$, will have a very predictable and reproducible collection of peptides produced by a trypsin digestion. Trypsin also produces a very manageable average peptide length of approximately 14 residues $^{114}$, suggesting that many of the produced peptides would be in a mass range most suitable for bottom-up proteomics. For example, the tryptic hemoglobin peptide containing $\mathrm{Hb} \beta^{93}$ Cys is GTFATLSELHCDK, containing just 13 amino acids. 


\subsubsection{LC-MS Peptide and Protein Analysis}

Peptides fragment in specific patterns following CID. These fragmentation patterns are well-documented in the literature and are relatively straight-forward with few exceptions. ${ }^{112}$ The fragmentation along the peptide backbone can be used to determine amino acid sequences and alterations (adduction) to the specific amino acids of a known sequence. As can be seen in Figure 8, the fragments are given the nomenclature of a,b,c (if the charged is maintained on the $\mathrm{N}$-terminus) or $\mathrm{x}, \mathrm{y}, \mathrm{z}$ (if the charge is maintained on the $\mathrm{C}$-terminus), depending upon the cleavage of the peptide bond. Of these formed fragments, the most commonly observed are the " $y$ " and "b" fragments, or the fragments formed by the cleavage of the peptic bond between the carbonyl group and the amine.

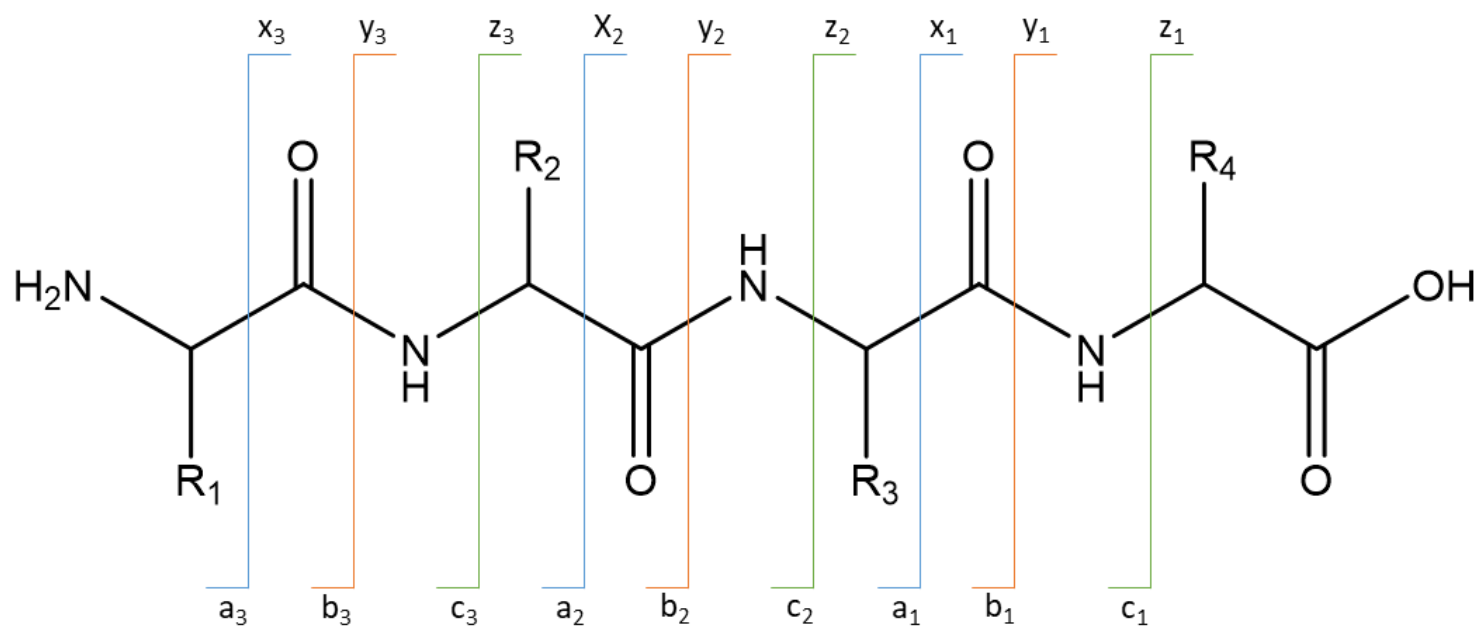

Figure 8. Peptide fragmentation patterns labeled with the common " $a, b, c$ " and " $x, y, z$ " nomenclature. 


\subsection{In Vitro Metabolic Assays}

The formation of drug metabolites in an in vitro system may be monitored by a metabolic assay system. Such metabolic assays mimic the natural metabolism processes a xenobiotic may undergo in the human body. ${ }^{45}$ In vitro metabolic assays are typically used in pharmaceutical development to characterize stable and reactive drug metabolites formed by natural metabolic processes found in the human body. ${ }^{45,115}$ The most common types of in vitro metabolic assays utilize homogenized liver fractions containing various enzymes responsible for metabolism. These fractions include the S9 fraction, cytosol, and human liver microsomes (HLM). The S9 fraction is the supernatant produced from initial low speed centrifugation of a liver homogenate. When the S9 fraction is then centrifuged at higher speed $(100 \times \mathrm{g})$, the supernatant consists of the cytosolic fraction while the pellet consists of "microsomes" derived from rough endoplasmic reticulum.. Human liver microsomes (HLM), the metabolic system utilized in this research, contains a high concentration of the CYP enzymes responsible for Phase I metabolism described in section 2.2.1.

\subsubsection{In Vitro Metabolic Trapping Assays}

As described previously, when certain xenobiotics are metabolized, reactive intermediates may also be formed, which may then modify nearby macromolecules to form covalent adducts, primarily through electrophilicnucleophilic interactions. ${ }^{116,117}$ The formation of these modifications can create a potential for organ-specific toxicity ${ }^{118,119}$ or, alternatively, can be innocuous. In

either case, such adducts may also serve as biomarkers of exposure. ${ }^{88}$ Metabolic 
trapping assays have been widely employed to study these possibly harmful products in vitro, particularly in pharmaceutical development where there is a need to identify the potential for reactive metabolite production in candidate drugs. ${ }^{89,120}$ Such assays are designed to mimic Phase I and II metabolic processes in human cells. ${ }^{45}$ When a metabolic assay is used for the purpose of examining reactive metabolite formation, a trapping agent must be added as a target for covalent modification. ${ }^{121}$ Trapping agents are typically any one of numerous, primarily nucleophilic and generally small, molecules that can bind covalently to reactive intermediates, preventing further metabolism and preserving the structure of the otherwise unstable compound. ${ }^{61}$ Examples of trapping agents used in these assays include glutathione $(\mathrm{GSH})^{89,122}, \mathrm{~N}-$ acetylcysteine ${ }^{61}$, and cyanide ${ }^{45}$, with the emphasis of this research being placed on GSH. Additionally, larger trapping agents may be used, such as larger peptides or whole proteins containing at least one nucleophilic site. 


\subsection{Research Objectives}

This research was performed to test three hypotheses:

1. In vitro metabolic assays can successfully mimic in vivo metabolic processes for relevant drugs of abuse.

2. Small peptides used as trapping agents in in vitro metabolic assays can form covalent adducts with reactive metabolites of drugs of abuse.

3. These same adducted moieties can also be observed on whole proteins. The primary objective of this research was to observe and characterize covalent adducts with hemoglobin by reactive metabolites of certain drugs of abuse. This objective was accomplished by completing the following three tasks.

1. Create and optimize a LC-MS/MS method for the detection and characterization of drugs of interest and stable drug metabolites. Specifically:

a. Create a LC-MS method to determine the retention time of the parent drugs.

b. Using retention times, create a targeted MS/MS method to determine and analyze common transitions for each parent drug.

c. Perform metabolic assays and identify and confirm metabolites formed under assay conditions via LC-MS/MS.

2. Create and optimize a LC-MS/MS method for the detection and characterization of reactive drug metabolites. Specifically:

a. Optimize a metabolic trapping assay using glutathione as a nucleophilic trapping agent. 
b. Analyze MS and MS/MS data collected from GSH studies to compile a list of possible adducts formed.

c. Determine plausible structures for observed adducts using MS and MS/MS data.

3. Confirm adduction in purified proteins by drugs of interest.

a. Optimize a metabolic trapping assay to incorporate hemoglobin as a trapping agent.

b. Analyze $\mathrm{Hb}$ trapping assays to determine sites of adduction and number of expected adducts.

c. Confirm adduction with $\mathrm{Hb}$ by collecting MS/MS data for the $\mathrm{Hb}$ tryptic peptides. 


\section{Methodology}

\subsection{Instrumentation}

Analysis by LC-QqQ-MS was performed on an Agilent 1290 Infinity ultrahigh performance liquid chromatography (UHPLC) coupled to an Agilent 6460 QqQ MS. Analysis by LC-QTOF-MS was performed on an Agilent 1290 Infinity UHPLC coupled to an Agilent 6530 QTOF MS. Both positive and negative ion scanning modes were used with the LC-QTOF-MS, while only negative mode ionization was used for the LC-QqQ-MS. Both instruments utilized Agilent Jet Stream electrospray ionization (ESI). The column utilized for both instruments was an Agilent Zorbax Eclipse Plus $\mathrm{C}_{18}$ rapid resolution HD. Elution solvents, injection volumes, and LC pump parameters varied for experiments and are discussed below. Elution solvent flow rate was $0.3 \mathrm{~mL} / \mathrm{min}$. for all runs. Data acquisition for the QqQ and QTOF was performed using Agilent's MassHunter Acquisition software (version B.06.00 for both systems). Data analysis was performed using Agilent's MassHunter Qualitative software (version B.07.00), with supplementation by Agilent's BioConfirm software (version B.08.00).

\subsection{Drug Selection}

The drugs of interest in this study were selected based on a known potential for addiction/dependence and prevalent usage, while also ensuring that various structural and pharmacological classes of drugs were represented in the final list. All of the selected drugs are examples of compounds which may be identified in authentic specimens from law enforcement cases, rehabilitation 
centers, correctional facilities, and outpatient therapy. As mentioned in section 2.1, the drugs of interest selected for this study were a-pyrrolidinopentiophenone, acetaminophen, alprazolam, buprenorphine, clozapine, cocaine, diazepam, methadone, methamphetamine, methylenedioxymethamphetamine, methylenedioxypyrovalerone, methylone, morphine, naltrexone, oxycodone, and $\Delta^{9}$-tetrahydrocannabinol. The two positive control compounds selected for this study are acetaminophen and clozapine, both of which have shown the capability of binding to endogenous nucleophiles. Radiolabeled acetaminophen has been shown to bind to the free cysteine in bovine serum albumin, with the adduct being identified as 3-cystein-S-yl-4-hydroxyaniline. ${ }^{123}$ Additional studies by James, et al. investigated the potential for liver induced toxicity by acetaminophen-protein adducts. ${ }^{124}$ McGill, et al. reported that acetaminophenprotein binding is possible at subtoxic levels of administration and before levels of glutathione, a generally more favorable target of electrophilic compounds, were depleted. ${ }^{125}$ Both acetaminophen and clozapine have been shown to exhibit affinities to covalently bind to glutathione on multiple occasions. ${ }^{110,126-128}$

\subsection{Parent Drug Analysis}

In order to determine the appropriate instrumental parameters for the analysis of the metabolites formed by the drugs of abuse, the parent drugs were analyzed for retention time and major fragments formed. Analysis of the parent drugs was performed using LC-QTOF-MS using positive ionization and full scan mode. Elution solvents for the parent drug analysis were A: 5 millimolar $(\mathrm{mM})$ 
ammonium formate in water with $0.1 \%$ formic acid and $\mathrm{B}$ : acetonitrile with $0.1 \%$ formic acid. Pump parameters were as follows: 0-2 min. 5\% B, $2-5$ min. ramp from $5 \%$ B to $50 \%$ B, $5-7$ min ramp from $50 \%$ B to $100 \%$ B, hold at $100 \%$ B until 10 min., followed by a two min. post-run for re-equilibration of the instrument to the starting configuration. A one part per million (ppm) solution in methanol was created for each of the 16 drugs used in this study (Table 2), except for APAP, which due to solubility issues was dissolved in water, and THC which was dissolved in ethanol. A $5 \mu \mathrm{L}$ aliquot of each drug-containing solution was injected into the mass spectrometer, and the corresponding retention time was collected.

Once retention times were collected for all parent drugs, mixtures were created containing three of the parent drugs with differing retention times. Targeted scan mode MS/MS analysis was performed on these five mixtures to collect major transitions using the same LC-MS parameters as the retention time collection. Transitions were collected at 10, 20, and 40 electron volts (eV) to ensure that complete fragmentation was achieved for all drugs. These scans were performed in triplicate, and the three consistently most prominent fragments were recorded.

\subsection{Drugs of Abuse Stable Metabolites}

\subsubsection{Stable Metabolite Formation}

Stable metabolites were analyzed for clozapine, which has prominently formed and well-established stable metabolites. ${ }^{129}$ These metabolites were studied to aid in the optimization of parameters for the in vitro metabolic assay 
required for later research. Briefly, the stable metabolite assay consisted of final concentrations of $100 \mu \mathrm{M}$ drug of interest, $1 \mathrm{mg} / \mathrm{mL}$ HLM, $2 \mathrm{mM}$ nicotinamide adenine dinucleotide phosphate (NADPH), $3 \mathrm{mM}$ magnesium chloride $\left(\mathrm{MgCl}_{2}\right), 3$ $\mathrm{mM}$ glucose-6-phosphate (G6P), and $0.4 \mathrm{U} / \mathrm{mL}$ glucose-6-phosphate dehydrogenase (G6PD) with a total assay volume of $125 \mu \mathrm{L}$ in $50 \mathrm{mM}$ sodium phosphate buffer $\mathrm{pH}=7.4$. A high drug concentration and the use of an activating cofactor regeneration system were employed to ensure maximum formation of metabolites. The assay components were combined in a microfuge tube and briefly vortexed to ensure uniformity. The vials were then incubated $a 7^{\circ} \mathrm{C}$ for two hours. Following incubation, enzymatic activity was ceased by the addition of $25 \mu \mathrm{L}$ ice-cold acetonitrile (6\% acetic acid). The vials were then centrifuged at $15,000 \times \mathrm{g}$ at $4^{\circ} \mathrm{C}$ for 30 minutes. After centrifugation, a $100 \mu \mathrm{L}$ aliquot was removed and placed into a clean LC-MS vial for analysis.

\subsubsection{Stable Metabolite Analysis}

Stable metabolite analysis was performed using LC-QTOF-MS using positive ionization and full scan mode. Elution solvents for the parent drug analysis were A: 5 millimolar $(\mathrm{mM})$ ammonium formate in water with $0.1 \%$ formic acid and B: acetonitrile with $0.1 \%$ formic acid. Injection volume was set to $1 \mu \mathrm{L}$. Pump parameters were as follows: $0-2 \mathrm{~min} .5 \% \mathrm{~B}, 2-5 \mathrm{~min}$. ramp from $5 \% \mathrm{~B}$ to $50 \%$ B, 5-7 min ramp from $50 \%$ B to $100 \%$ B, hold at $100 \%$ B until 10 min., followed by a two min. post-run for re-equilibration of the instrument to the starting configuration. Collected spectra were compared to negative controls without a metabolic system, and the new peaks were analyzed. 


\subsection{Metabolic Trapping Assay with Glutathione}

Once the in vitro assay parameters were optimized, adduction by reactive metabolites with GSH was studied. This process required a similar in vitro assay as the stable metabolites, but with the addition of a trapping agent. Briefly, components of the in vitro trapping assay were combined in a total assay volume of $125 \mu \mathrm{L}$ of $50 \mathrm{mM}$ sodium phosphate buffer, $\mathrm{pH} 7.4$, at the following final concentrations: $1000 \mu \mathrm{M}$ drug of interest, $1 \mathrm{mg} / \mathrm{mL} \mathrm{HLM}, 3 \mathrm{mM} \mathrm{MgCl} 2,2 \mathrm{mM}$ $\mathrm{NADPH}, 3 \mathrm{mM} \mathrm{G6P}, 0.4 \mathrm{U} / \mathrm{mL}$ G6PD, and $2 \mathrm{mM} \mathrm{GSH}$. Drug of interest is added to a clean microfuge tube and solvent is removed via vacufuge. Assay components without GSH were then combined in the microfuge vial and vortexed briefly to ensure uniformity, followed by a pre-incubation of $15 \mathrm{~min}$ at $37^{\circ} \mathrm{C}$. The GSH was then added to complete the assay and achieve final assay volume, and vials were once again vortexed to ensure proper mixing. Incubation then ensued at $37^{\circ} \mathrm{C}$ for $3 \mathrm{~h}$. Upon completion of incubation, vials were immediately centrifuged at $15,000 \times \mathrm{g}$ at $4^{\circ} \mathrm{C}$ for $30 \mathrm{~min}$. Following centrifugation, $100 \mu \mathrm{L}$ aliquots of supernatant were removed from each vial and placed in separate, clean LC vials. This assay method is illustrated in Figure 9. 
Drug is added to a clean microfuge vial. Human liver microsomes, $\mathrm{NADPH}, \mathrm{MgCl}_{2}$, glucose-6-phosphate G-6-P dehydrogenase, all in sodium phosphate buffer, are added to the vial.

Following centrifugation, supernatant is removed and added to a clean LC-MS vial for analysis.

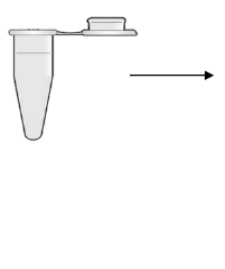

Vials are pre-incubated at $37^{\circ} \mathrm{C}$ for $15 \mathrm{~min}$

GSH is added, and vials are incubated at $37^{\circ} \mathrm{C}$ for $3 \mathrm{~h}$.

Following incubation, vials are centrifuged at $15,000 \times \mathrm{g}$ at $4^{\circ} \mathrm{C}$ for $30 \mathrm{~min}$.

Figure 9. Illustration of the metabolic trapping assay method utilizing GSH as a trapping agent.

\subsubsection{Glutathione Adduct Analysis}

Preliminary analysis of GSH adducts was performed using positive ionization and full scan mode on the LC-QTOF-MS. Elution solvents for the parent drug analysis were A: 5 millimolar ( $\mathrm{mM}$ ) ammonium formate in water with $0.1 \%$ formic acid and B: acetonitrile with $0.1 \%$ formic acid. Injection volume was set to $1 \mu \mathrm{L}$. Pump parameters were as follows: $0-2$ min. $5 \%$ B, $2-5$ min. ramp from $5 \%$ B to $50 \%$ B, $5-7$ min ramp from $50 \%$ B to $100 \%$ B, hold at $100 \%$ B until 10 min., followed by a two min. post-run for re-equilibration of the instrument to the starting configuration.

Negative mode analysis work began with the LC-QQQ-MS and used PIS. Elution solvents for GSH adduct analysis in negative mode were A: water with $0.1 \%$ acetic acid, and B: $95 \%$ acetonitrile, $4.9 \%$ water, and $0.1 \%$ acetic acid. The pump timetable was as follows: $5 \% \mathrm{~B}$ hold for $1 \mathrm{~min}, 5 \%$ to $100 \% \mathrm{~B}$ ramp from 1 to $14 \mathrm{~min}, 100 \%$ B hold for $2 \mathrm{~min}$, followed by a 3-min post-time for reequilibration to initial conditions for next injection. The precursor ion scan was 
set to identify the precursor ions for any fragments with $\mathrm{m} / \mathrm{z} 272$, a common and indicative product of GSH, corresponding to GSH less the sulfur atom. ${ }^{110}$ The scan took place over the mass range $\mathrm{m} / \mathrm{z} 400-800$ to avoid interference from unreacted GSH ([M-H] $m / z$ 306) and to maximize detection of likely adducts for all drugs. Peaks were determined to be potentially significant if they had an unscaled abundance higher than 1000 counts. All peaks determined to be potentially significant for each drug had their $\mathrm{m} / \mathrm{z}$ recorded. The extracted ion chromatogram (XIC) MS spectrum was also collected for each potentially significant peak. The XIC was examined for characteristic fragmentation common to GSH-containing compounds.

\subsubsection{Glutathione Adduct Confirmation}

Negative mode analysis was continued on the LC-QTOF-MS. Initial analyses by QTOF-MS were performed using full scan mode. In full scan mode, the mass range was again restricted to $\mathrm{m} / \mathrm{z} 400-800$ with fragmentor voltage set to $120 \mathrm{~V}$ and no collision induced dissociation. Data were collected for this mass range over the entire run time and any prominent peaks of interest were recorded and then analyzed using targeted MS/MS. In targeted mode, MS/MS data were collected only for the molecular ion peaks of interest, with the mass range set to $m / z 100-800$ to allow for smaller identifying fragments to be recorded at collision energies of 10,20 , and $40 \mathrm{eV}$ to allow for full visualization of fragments formed. Compounds with fragmentation consistent with masses commonly seen for fragmentation of GSH were recorded and compared to the list previously compiled from the initial QQQ-MS analyses. In addition, several putative adducts 
not seen during initial low-resolution MS analysis were observed by QTOF-MS. The compounds deemed of interest also showed the GSH-characteristic ion with the mass of $m / z\left(\mathrm{M}-\mathrm{H}^{+}\right)^{-}-272$. As with the QQQ-MS studies, other ions previously reported to be characteristic of GSH adducts in negative mode ESI were typically present.

\subsection{Identification of Glutathione Adduct Structures}

Adduct structures were proposed based on accurate mass data for the molecular ion of each drug-GSH adduct and for major MS/MS fragments. Masses consistent with previously reported adducts were assigned the respective structures published in the literature. For novel adducts, a list of metabolites potentially formed in situ was compiled using published metabolism data as available and, where not available, the structure of the parent drug was altered using common metabolic processes via ChemDraw Prime software (PerkinElmer, version 16.0) until a plausible adduct structure was created. For this process, metabolic reactions that were examined included hydroxylation (along with NIH shift), $\mathrm{O}$ - and $\mathrm{N}$-demethylation, oxidation/reduction, and loss of reactive moieties, as these are common for Phase I metabolic pathways. ${ }^{61}$ Structures associated with more than one metabolic transformation were also considered. The final theoretical adduct list therefore consisted of multiple target structures for each drug.

Calculated molecular ion masses of the theoretical adduct structures were then compared to those observed in the QTOF-MS/MS analysis for each drug to 
identify tentative positive hits. For these compounds, MS/MS fragmentation data were then utilized for further confirmation of adduct structure. Both GSH- and

drug-specific fragments were considered in this analysis for maximum confidence in the resultant structural assignments. Where present, exact stereo- and regiospecificity of the covalent adduct bond was not identified.

\subsection{Adduction with Hemoglobin}

The metabolic trapping assay with $\mathrm{Hb}$ as a trapping agent required some initial optimization. The first utilized assay method involved a cup insert for microfuge tubes with a dialysis membrane in the bottom, allowing for passive diffusion of smaller molecules. These dialysis membrane cups had not been used for this purpose, but were explored as an option because it would allow for full separation of the HLM enzymes and the $\mathrm{Hb}$ protein without the need for additional separation.

\subsubsection{Hemoglobin Trapping Assay Method}

Adduction with hemoglobin was performed using a trapping assay similar to the GSH trapping assay followed by proteolytic digestion by trypsin to produce smaller peptides. Briefly, components of the in vitro trapping assay were combined in a total assay volume of $250 \mu \mathrm{L}$ of $25 \mathrm{mM}$ sodium phosphate buffer, $\mathrm{pH} 7.4$, at the following final concentrations: $500 \mu \mathrm{M}$ test drug, $0.5 \mathrm{mg} / \mathrm{mL} H \mathrm{HLM}, 1$ mM NADPH, $1.5 \mathrm{mM} \mathrm{MgCl} 2,1.5 \mathrm{mM}$ G6P, 0.2 units $/ \mathrm{mL} \mathrm{G6PD} \mathrm{and} 2.5 \mathrm{mg} / \mathrm{mL}$ of a prepared human $\mathrm{Hb}$ solution $(5 \mathrm{mg} / \mathrm{mL} \mathrm{Hb}$ in $25 \mathrm{mM}$ ammonium bicarbonate buffer). Drug of interest is added to a clean microfuge tube and solvent is 
removed via vacufuge. Assay components were then combined in the microfuge vial and vortexed briefly to ensure uniformity, the tube was then incubated with agitation at $37^{\circ} \mathrm{C}$ for $6 \mathrm{~h}$. Following incubation, the vial was centrifuged for $30 \mathrm{~min}$ at $100,000 \times \mathrm{g}$ at $4^{\circ} \mathrm{C}$. After centrifugation, the supernatant was transferred into a clean microfuge tube. A freshly-prepared $15 \mathrm{mM}$ solution of iodoacetamide (IAM) was then added, and the vial was incubated at room temperature in the dark for $1 \mathrm{hr}$. Following incubation, intact $\mathrm{Hb}$ was extracted from the reaction mixture via Amicon® Ultra $0.5 \mathrm{~mL}$ centrifugal filter spun at $15,000 \mathrm{xg}$ for $10 \mathrm{~min}$. $\mathrm{Hb}$ was then collected into a new clean microfuge vial and reconstituted in a 1 $\mathrm{mg} / \mathrm{mL}$ solution of $\mathrm{CaCl}_{2}$. A $0.25 \mathrm{mg} / \mathrm{mL}$ solution of trypsin was then added, and the vial was incubated at $37^{\circ} \mathrm{C}$ for $16 \mathrm{~h}$. The vial was then centrifuged for $30 \mathrm{~min}$ at $100,000 \times \mathrm{g}$ at $4^{\circ} \mathrm{C}$, and the supernatant collected and placed into a clean LCMS vial for analysis. An illustration of this metabolic trapping assay method is found in Figure 10.

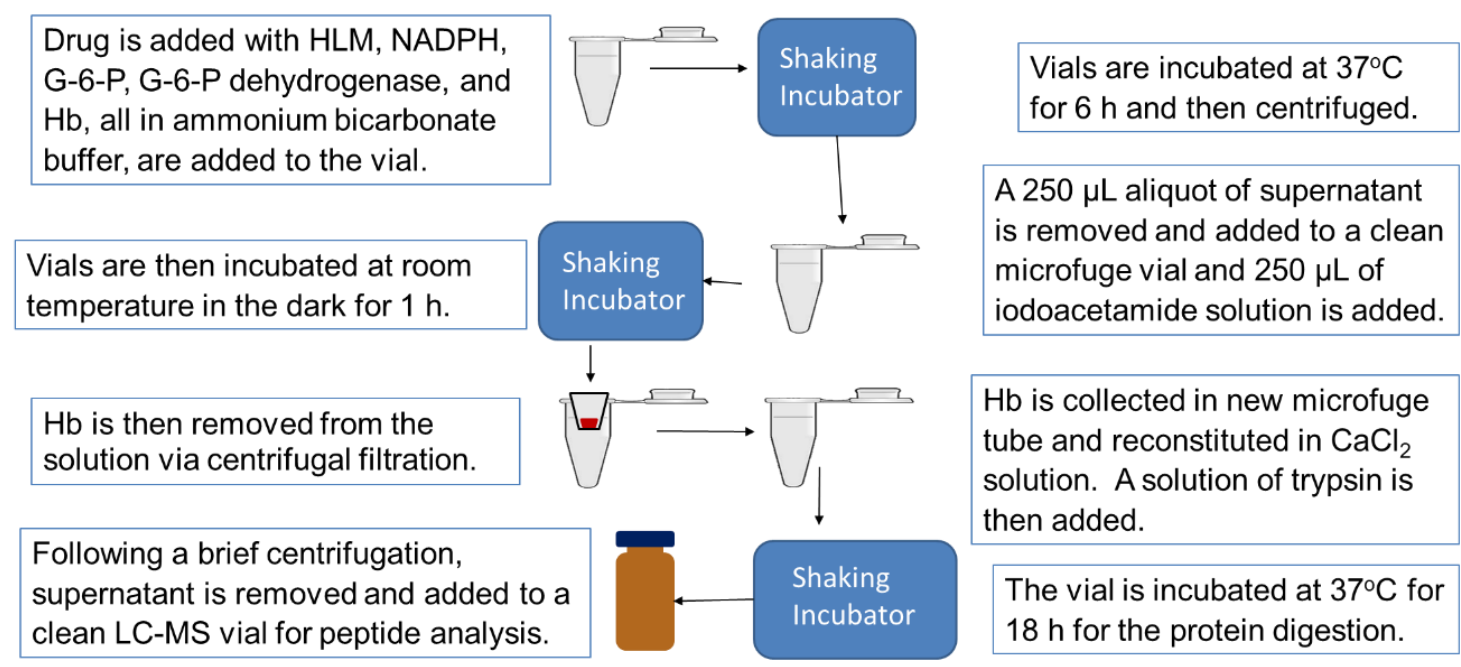

Figure 10. Illustration of the metabolic trapping assay method utilizing $\mathrm{Hb}$ as a trapping agent. 


\subsubsection{Hemoglobin Adduct Analysis}

Analysis of the tryptic peptides was performed using LC-QTOF-MS

utilizing first full scan mode followed by Auto MS/MS scan mode with positive ionization. The full scan mode consisted of no collision energy and only collected MS data. The collision energy for the Auto MS/MS analysis was set using the equation $y=2 x+20$, where $y$ is the collision energy and $x$ is the mass of the parent ion. The masses 121.0388 and 922.0098 were excluded from collection because they are the masses of the reference ions used to maintain mass accuracy by the instrument. The following parameters were used for both scan mode analyses. Column temperature was controlled at $40^{\circ} \mathrm{C}$. Elution solvents for the tryptic peptide analysis were $A$ : water with $0.1 \%$ trifluoroacetic acid and $B$ : acetonitrile with $4.9 \%$ water and $0.1 \%$ trifluoroacetic acid. Injection volume was set to $3 \mu \mathrm{L}$. Pump parameters were as follows: $0-0.5 \mathrm{~min} .10 \% \mathrm{~B}, 0.5-18 \mathrm{~min}$. ramp from $5 \%$ B to $50 \%$ B, 18-20 min ramp from $50 \%$ B to $100 \%$ B, hold at $100 \%$ B until 21 min., followed by a two min. post-run for re-equilibration of the instrument to the starting configuration.

Analysis of the data collected for the peptide adducts was performed using Agilent's MassHunter BioConfirm software (version B.08.00). The workflow used was the "peptide digest" method, the condition was "reduced", the sequences selected were the subunits $\mathrm{Hba}$ and $\mathrm{Hb} \beta$, the enzyme selected was trypsin, and the modifications were acetylation, hydroxylation, and any appropriate custom modifications. The custom modifications were individually created for each drug of interest based upon observed adducts from GSH studies and, where GSH 
data were unavailable, data collected from adduction studies using a synthetic peptide (manuscript in progress by Moller, et al). For the MS studies, once the workflow was run the predicted modifications were analyzed, and any potential drug adducts were recorded. For the Auto MS/MS studies, the spectra were collected, compared to the theoretical peak list supplied by Protein Prospector, and confirmed peaks were recorded. Peaks were considered a close match if the observed mass was within 0.1 Daltons (Da) of the hypothetical mass reported in the theoretical peak table. Peaks were considered a possible match if the mass differential was $<0.5 \mathrm{Da}$. All peaks with a mass differential $\geq 0.5 \mathrm{Da}$ were excluded. The workflow for the analysis of the $\mathrm{Hb}$ peptide adducts can be found in Figure 11.

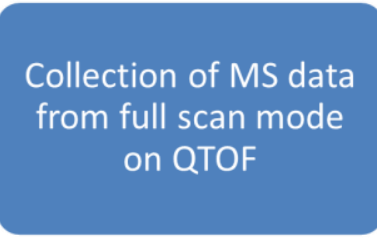

Determination of

potential adduct

masses for various

drugs via BioConfirm

Comparison of data

against a theoretical

peak table produced

by Protein Prospector
Reanalysis of drugs

utilizing Auto MS/MS

scan mode

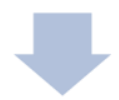

Collection of MS/MS

data via BioConfirm

for drug adducts when available

Figure 11. Flowchart of workflow for $\mathrm{Hb}$ peptide adduct analysis. 


\section{Results}

\subsection{Task 1: Create and optimize a LC-MS/MS method for the detection and characterization of drugs of interest and stable drug metabolites.}

\subsubsection{Parent Drug Retention Times}

Optimization of the acquisition method to analyze retention times of the parent drugs ensured that a single method could be used for the analysis of all of the drugs of interest. Solutions of individual drugs were injected into the instrument and the exact mass $\left([\mathrm{M}+\mathrm{H}]^{+}\right)$and retention times were recorded (Table 7). The overlaid total ion chromatograms (TICs) of 13 of the drugs of interest can be found in Figure 12. Several of the compounds had retention times very similar to others, and these drugs were separated into different mixtures for the next step of this task. The drugs of interest missing from Figure 12 were omitted because their TICs were recorded on a different date than the 13 drugs pictured. The positive controls APAP and CLZ were recorded separately (Figure 13), and THC needed to be dissolved in ethanol (Figure 14). Additionally, THC could not be identified as a peak without the use of the Find by Formula feature of the MassHunter Qualitative software, which targets the specific mass of a chemical formula and extracts corresponding peaks from the TIC. This method was utilized because THC exhibited poor ionization in positive mode, and coeluted with much of the material being flushed through the column by the high organic percentage of mobile phase at the end of the run (Figure 14). Because of these issues with the analysis of THC, the extracted ion chromatogram (XIC) for 
THC was utilized to determine the retention time, which was then added to the complete list of the drugs.

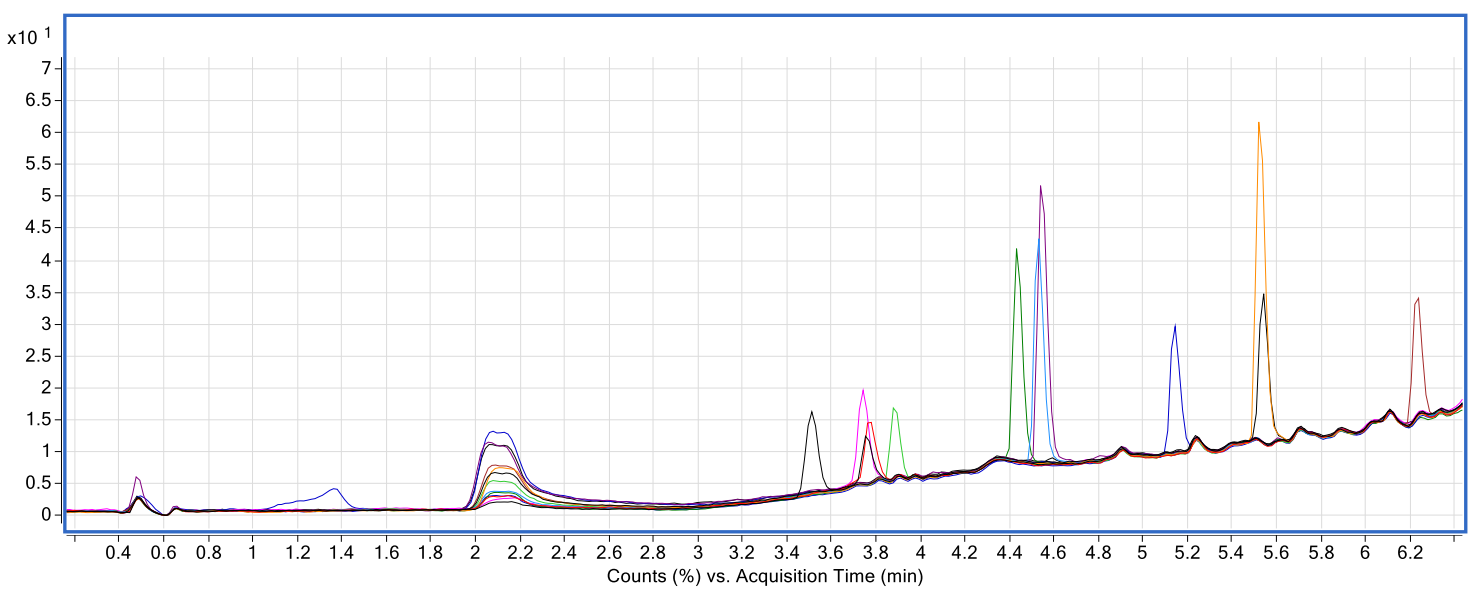

Figure 12. A zoomed in portion of the overlaid TICs for $\alpha$-PVP, ALP, BUP, COC, DZP, MDMA, MDPV, META, METH, METY, MOR, NAL and OXY. The peak at approximately 2.1 min. found in every spectrum is carryover from APAP.

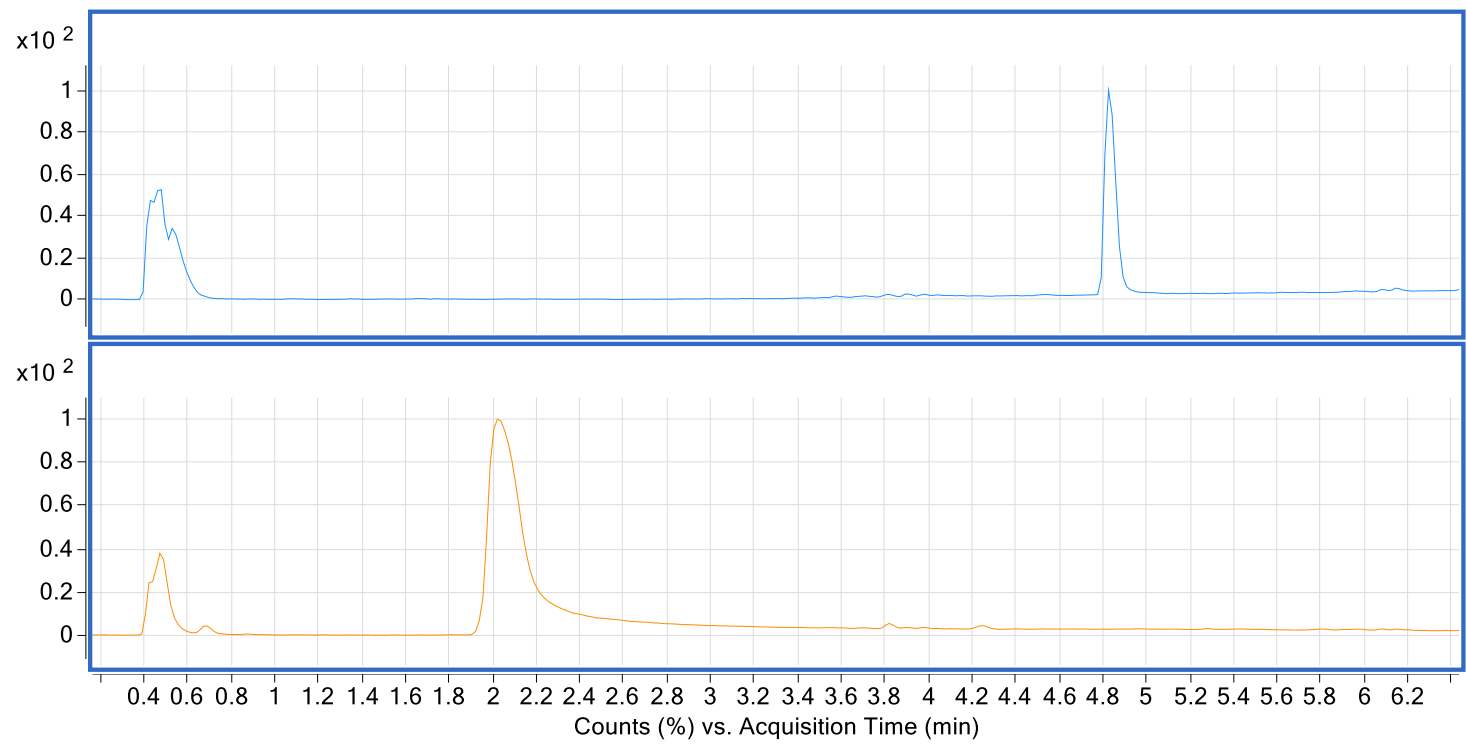

Figure 13. TICs for CLZ (top) and APAP (bottom), the two positive controls, used to collect their respective retention times. 


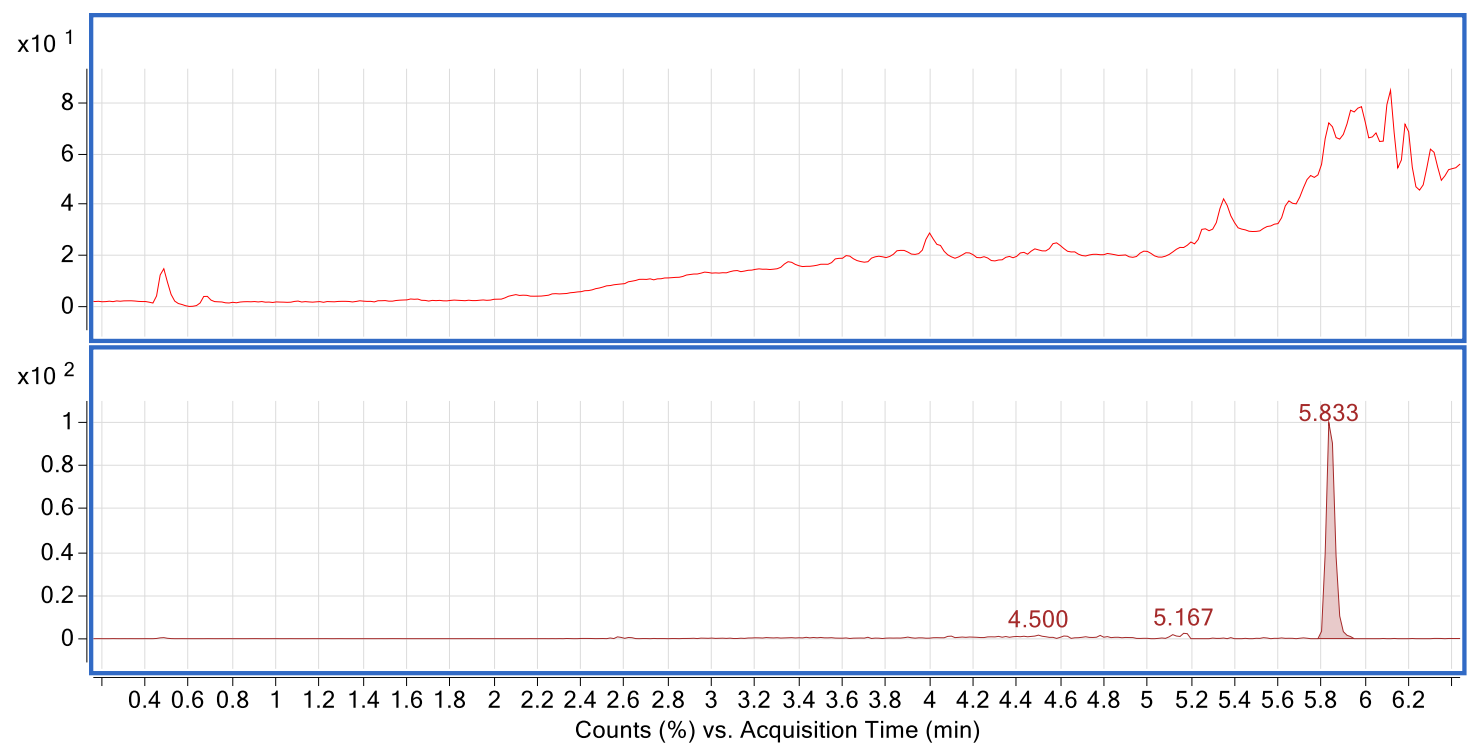

Figure 14. TIC (top) and XIC (bottom) for THC, showing the retention time for THC.

\subsubsection{Parent Drug Fragmentation}

After the collection of all retention times and exact masses observed by the instrument for each parent drug, the five mixtures of non-coeluting parent drugs were analyzed. The instrumental method used for analysis of the mixtures utilized targeted MS/MS mode, but all other parameters remained the same. The mixtures created can be found in Table 6.

\begin{tabular}{|l|l|}
\hline Mixture & Drugs Included \\
\hline Mixture \#1 & APAP, METH, COC \\
\hline Mixture \#2 & MDMA, a-PVP, DZP \\
\hline Mixture \#3 & META, METY, MDPV \\
\hline Mixture \#4 & ALP, MOR, OXY \\
\hline Mixture \#5 & BUP, NAL, THC \\
\hline
\end{tabular}

Table 6. Mixtures of parent drugs created to collect transitions 
CLZ was not included in the original mixtures, but was run individually. The TIC for each mixture (Figure 15) was analyzed using the Find by Targeted MS/MS feature on Masshunter Qualitative software. The collected spectra for each collision energy $(10,20$, or $40 \mathrm{eV})$ were analyzed separately to ensure that a complete list of fragments could be identified. It was necessary to collect these data so it would be possible to apply the same fragmentation patterns to proposed adducts at later stages of this research. The MS/MS data collected for the parent drugs can be found below in Figure 16. Table 7 was created by combining the information from the retention time experiments and the transition experiments for all of the drugs of interest. Many drugs had transitions with the same $\mathrm{m} / \mathrm{z}$, but are distinguishable by the other recorded transitions or retention times. 


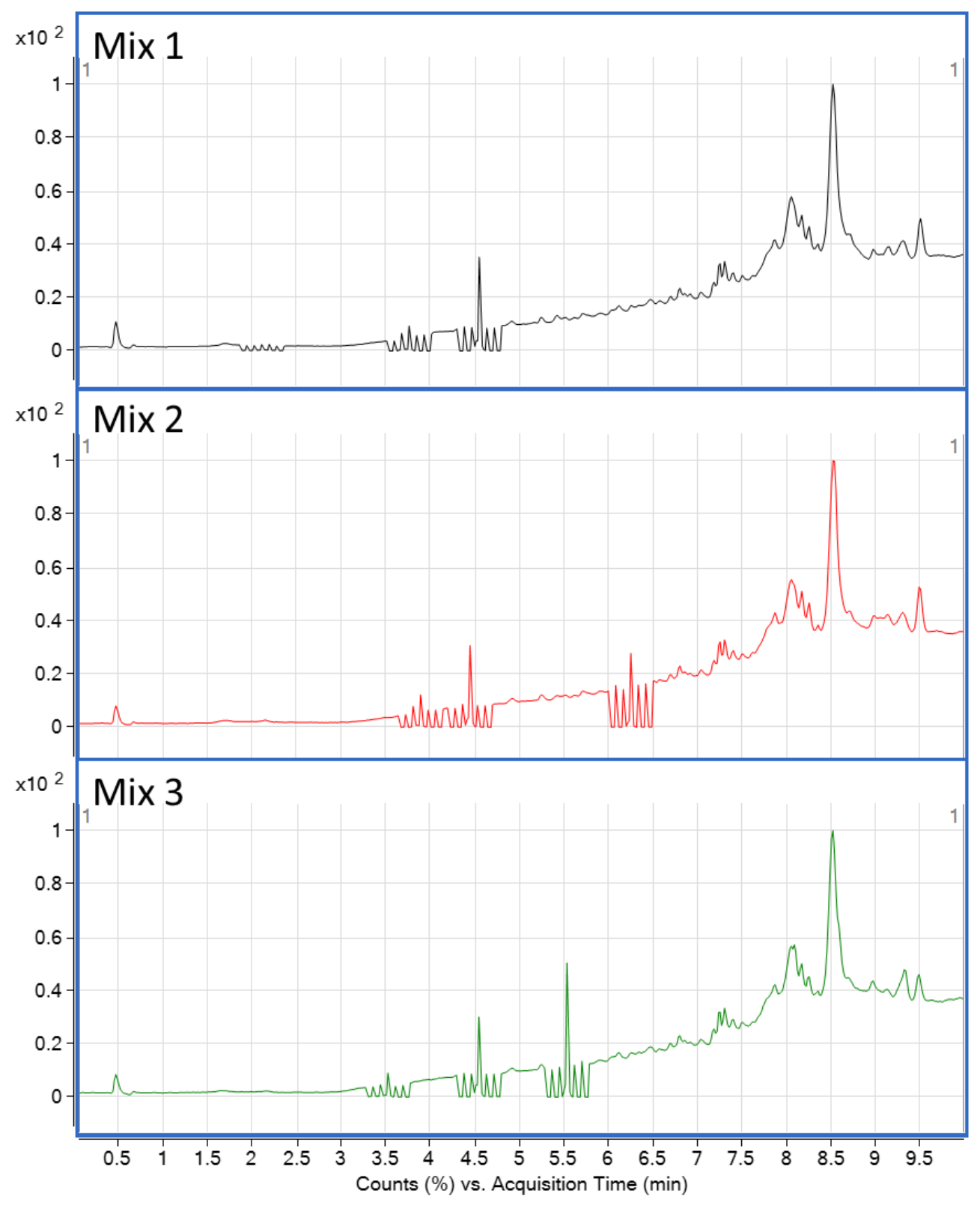




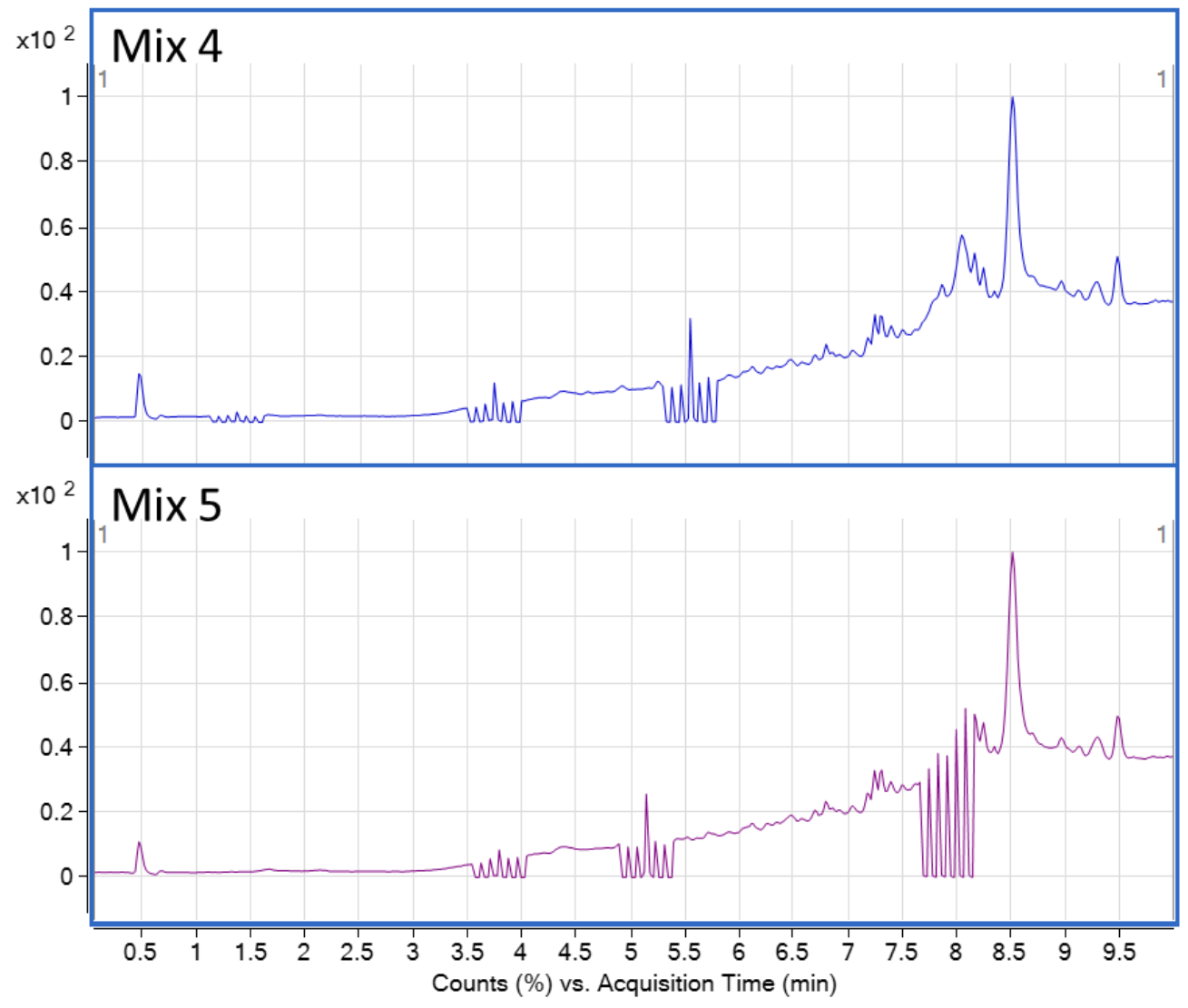

Figure 15. Targeted MS/MS TICs for the five mixtures of the parent drugs. 


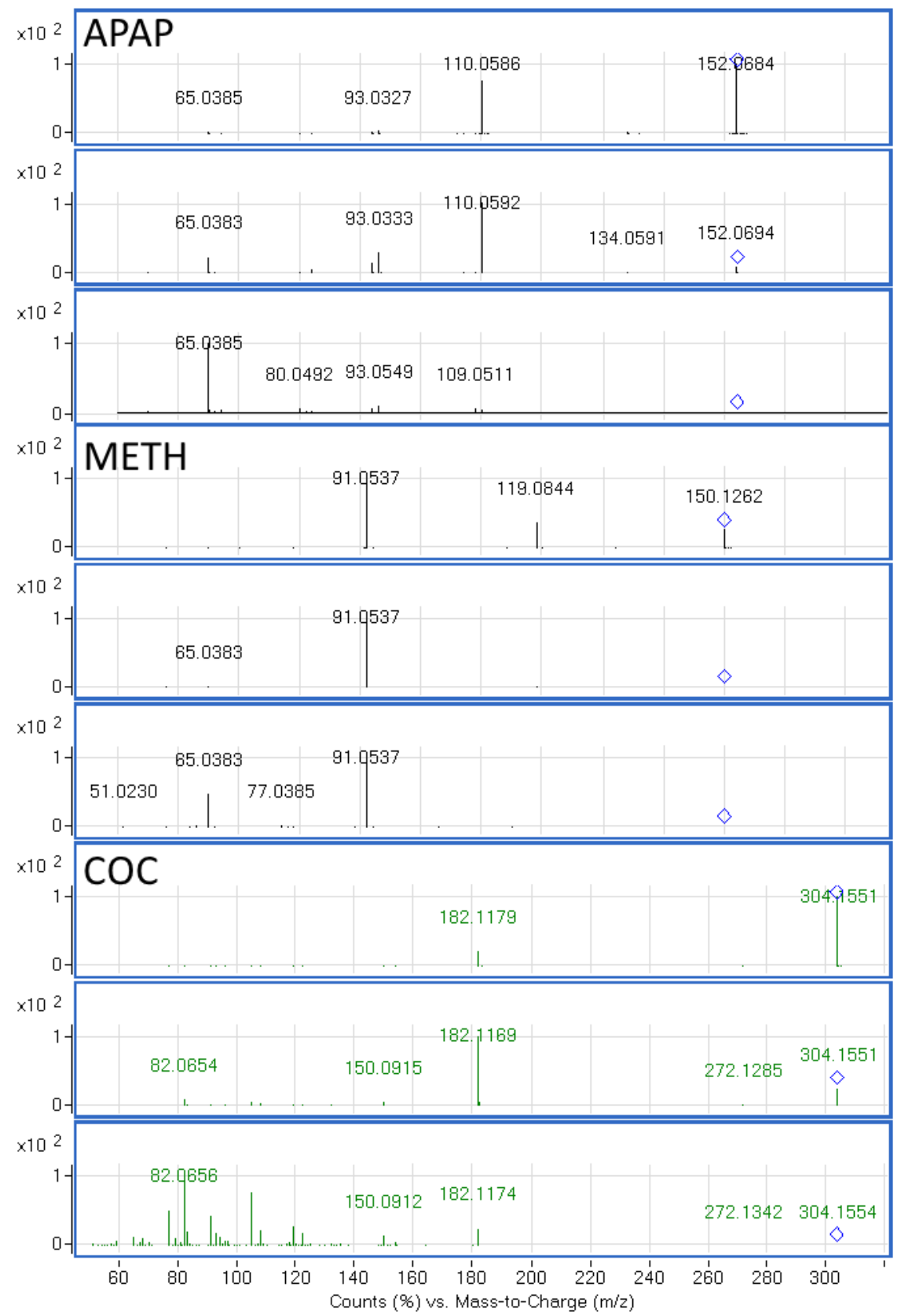




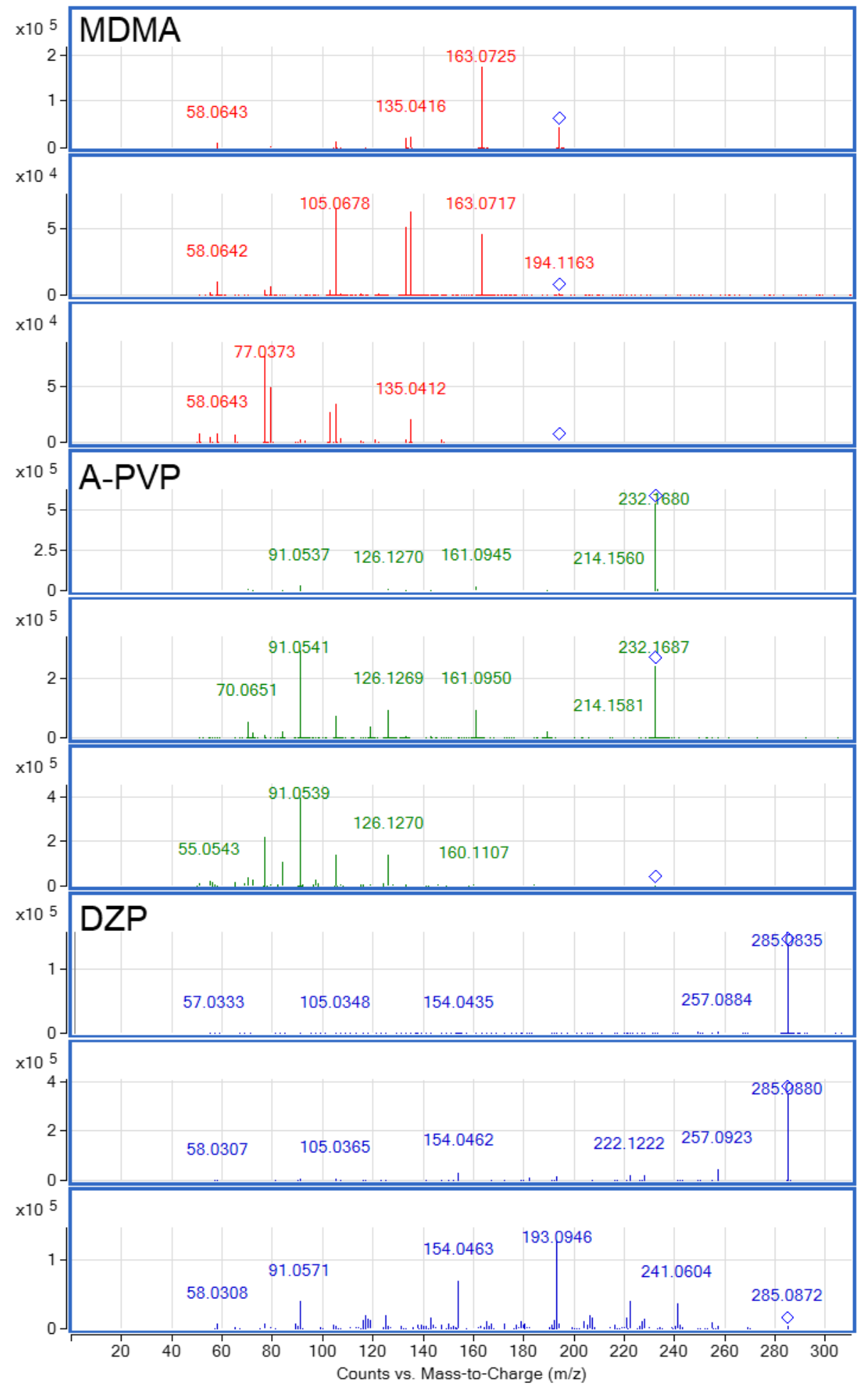



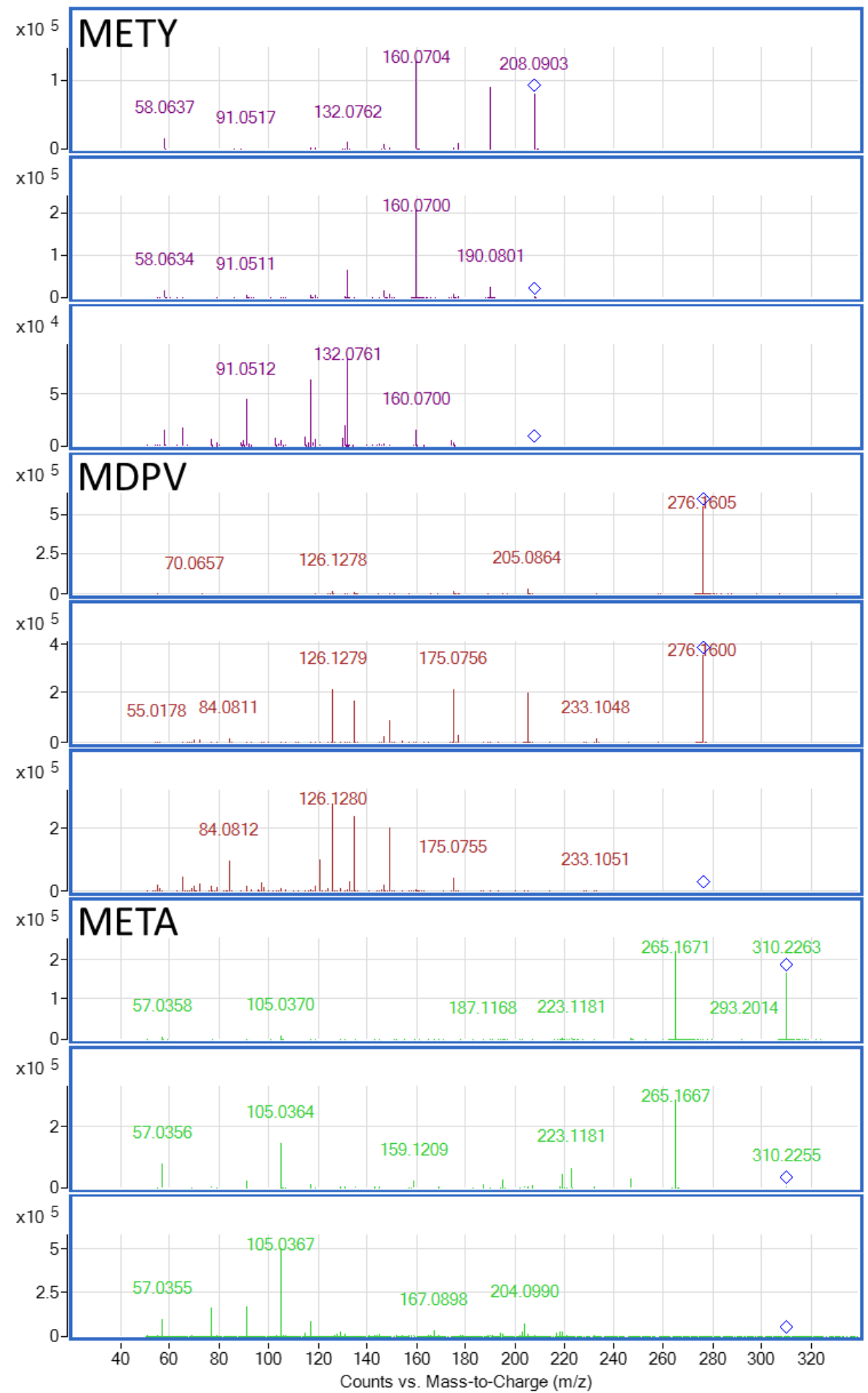


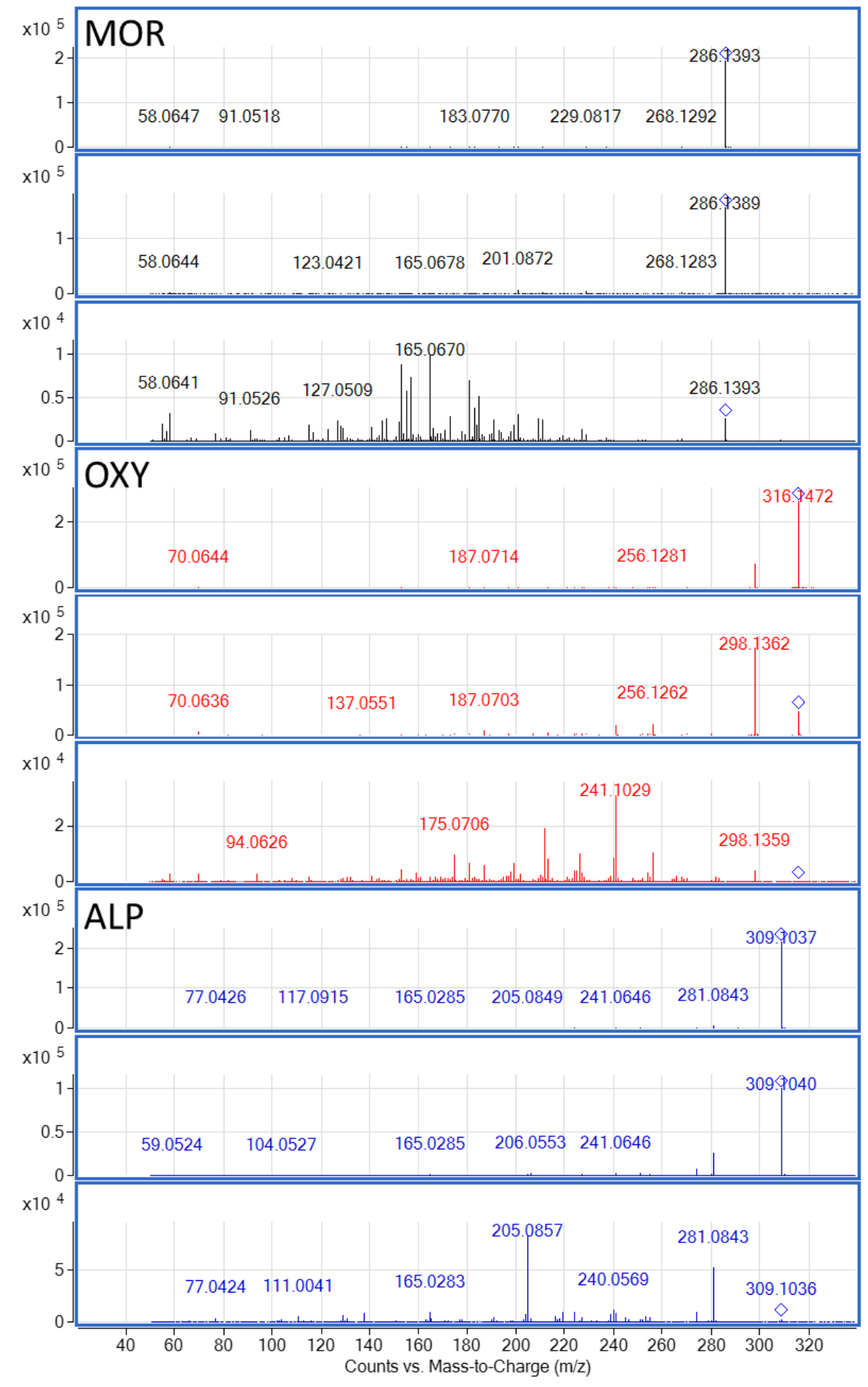




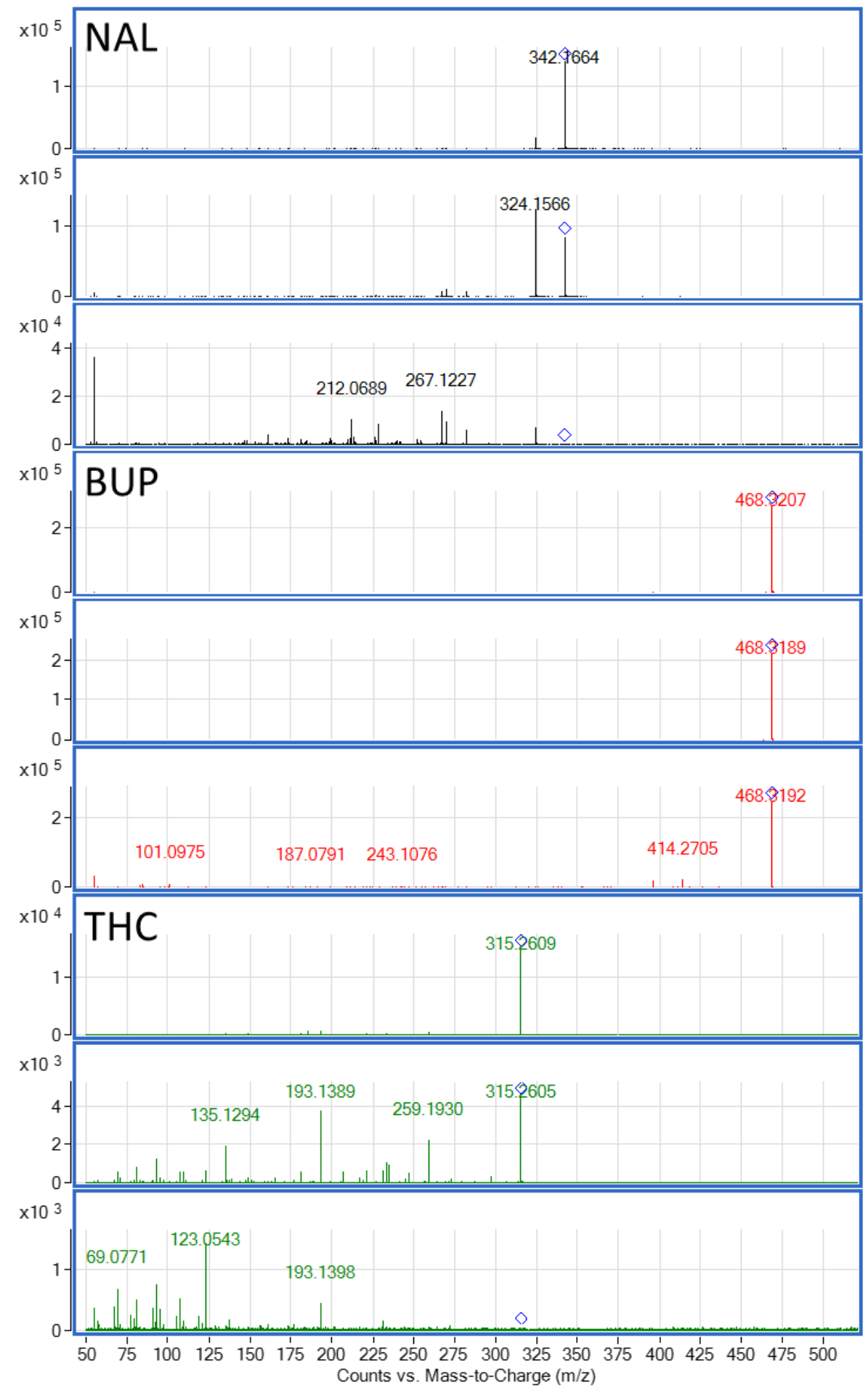

Figure 16. MS/MS data for the parent drugs. The data are shown at $10 \mathrm{eV}$ (top), $20 \mathrm{eV}$ (middle), and $40 \mathrm{eV}$ (bottom) for each drug. 
Formula $[\mathrm{M}+\mathrm{H}]^{+} \quad$ Ret. Time $\quad$ Tran. $1 \quad$ Tran. $2 \quad$ Tran. 3

\begin{tabular}{|c|c|c|c|c|c|c|}
\hline$\alpha-P V P$ & $\mathrm{C}_{15} \mathrm{H}_{21} \mathrm{NO}$ & 232.170 & 4.48 & 161.094 & 126.126 & 91.053 \\
\hline APAP & $\mathrm{C}_{8} \mathrm{H}_{9} \mathrm{NO}_{2}$ & 152.071 & 2.14 & 110.060 & 93.034 & 65.039 \\
\hline$A L P$ & $\mathrm{C}_{17} \mathrm{H}_{13} \mathrm{CIN}_{4}$ & 309.090 & 5.58 & 281.070 & 274.117 & 205.073 \\
\hline BUP & $\mathrm{C}_{29} \mathrm{H}_{41} \mathrm{NO}_{4}$ & 468.311 & 5.18 & 414.257 & 396.213 & 55.054 \\
\hline$C L Z$ & $\mathrm{C}_{18} \mathrm{H}_{19} \mathrm{CIN}_{4}$ & 327.147 & 4.88 & 270.089 & 227.045 & 192.075 \\
\hline$C O C$ & $\mathrm{C}_{17} \mathrm{H}_{21} \mathrm{NO}_{4}$ & 304.154 & 4.59 & 182.115 & 105.033 & 82.064 \\
\hline$D Z P$ & $\mathrm{C}_{16} \mathrm{H}_{13} \mathrm{CIN} \mathrm{N}_{2} \mathrm{O}$ & 285.079 & 6.28 & 193.089 & 154.042 & 91.054 \\
\hline MDMA & $\mathrm{C}_{11} \mathrm{H}_{15} \mathrm{NO}_{2}$ & 194.118 & 3.93 & 163.074 & 135.043 & 77.038 \\
\hline MDPV & $\mathrm{C}_{16} \mathrm{H}_{21} \mathrm{NO}_{3}$ & 276.159 & 4.58 & 205.083 & 175.072 & 126.126 \\
\hline META & $\mathrm{C}_{21} \mathrm{H}_{27} \mathrm{NO}$ & 310.217 & 5.57 & 265.156 & 105.033 & 77.038 \\
\hline METH & $\mathrm{C}_{10} \mathrm{H}_{15} \mathrm{~N}$ & 150.128 & 3.79 & 119.083 & 91.053 & 65.038 \\
\hline METY & $\mathrm{C}_{11} \mathrm{H}_{13} \mathrm{NO}_{3}$ & 208.097 & 3.56 & 190.085 & 160.075 & 132.080 \\
\hline MOR & $\mathrm{C}_{17} \mathrm{H}_{19} \mathrm{NO}_{3}$ & 286.144 & 1.41 & 229.085 & 201.090 & 165.069 \\
\hline NAL & $\mathrm{C}_{20} \mathrm{H}_{23} \mathrm{NO}_{4}$ & 342.170 & 3.82 & 324.156 & 267.112 & 55.054 \\
\hline$O X Y$ & $\mathrm{C}_{18} \mathrm{H}_{21} \mathrm{NO}_{4}$ & 316.154 & 3.78 & 298.141 & 256.132 & 241.108 \\
\hline$T H C$ & $\mathrm{C}_{21} \mathrm{H}_{30} \mathrm{O}_{2}$ & 315.232 & 7.78 & 259.174 & 193.126 & 135.119 \\
\hline
\end{tabular}

Table 7. Retention time and fragmentation data collected for all 16 drugs of interest in this research. Retention time is in minutes. Transitions collected were the three most prominent across all collision energies.

\subsubsection{Stable Drug Metabolites}

Attempts to simplify the metabolic assay used for the stable drug 
metabolites involved excluding the regeneration system for the NADPH. Initial experiments analyzing the stable metabolic assay did not utilize G6P and G6PD, and therefore produced poor results. The addition of the regeneration system yielded significantly better results and were then applied to the metabolic assay with clozapine.

When utilizing clozapine for metabolism studies, there are generally two major metabolites produced, clozapine $\mathrm{N}$-oxide and $\mathrm{N}$-desmethylclozapine (Figure 17). ${ }^{129}$ Following the in vitro metabolic assay performed, there were two peaks present in the TIC which were not present in the negative controls (Figure 18). Both of these new peaks corresponded to the major metabolites for clozapine, with $\mathrm{m} / \mathrm{z}$ of 343.1182 for clozapine $\mathrm{N}$-oxide and 313.1220 for $\mathrm{N}$ desmethylclozapine. Their XIC data can be seen in Figure 19.

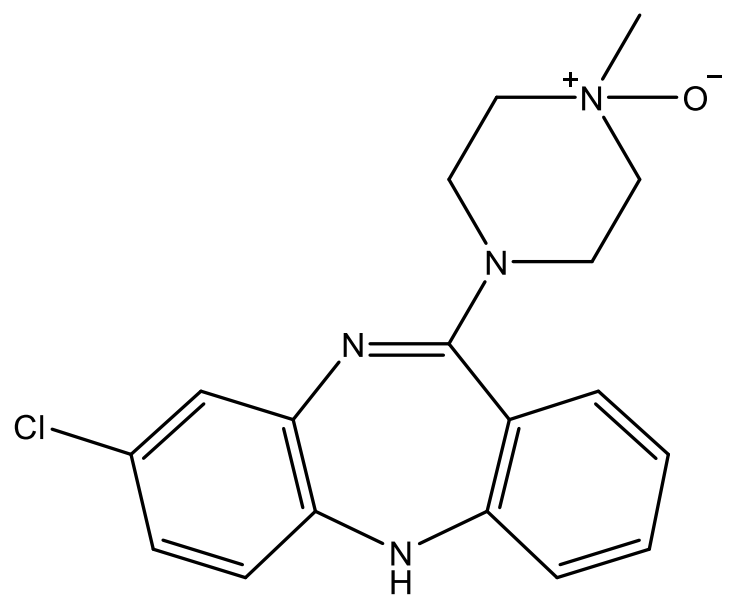

Exact Mass: 342.12

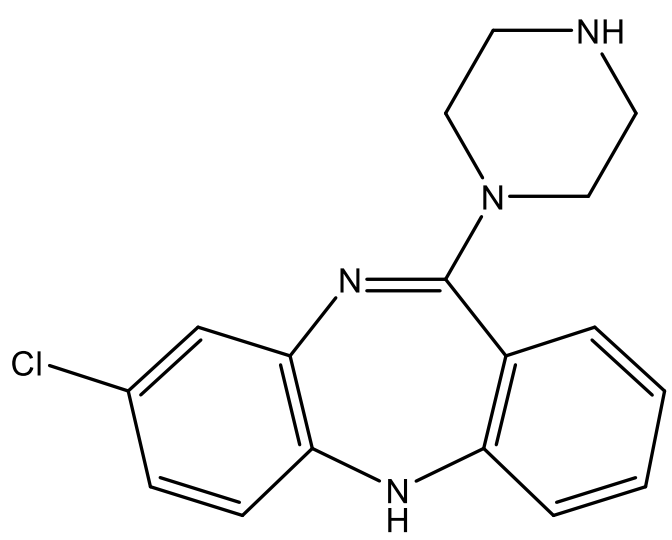

Exact Mass: 312.11

Figure 17. Two major metabolites for clozapine, clozapine $\mathrm{N}$-oxide (left) and $\mathrm{N}$ desmethylclozapine (right) with their exact masses. 


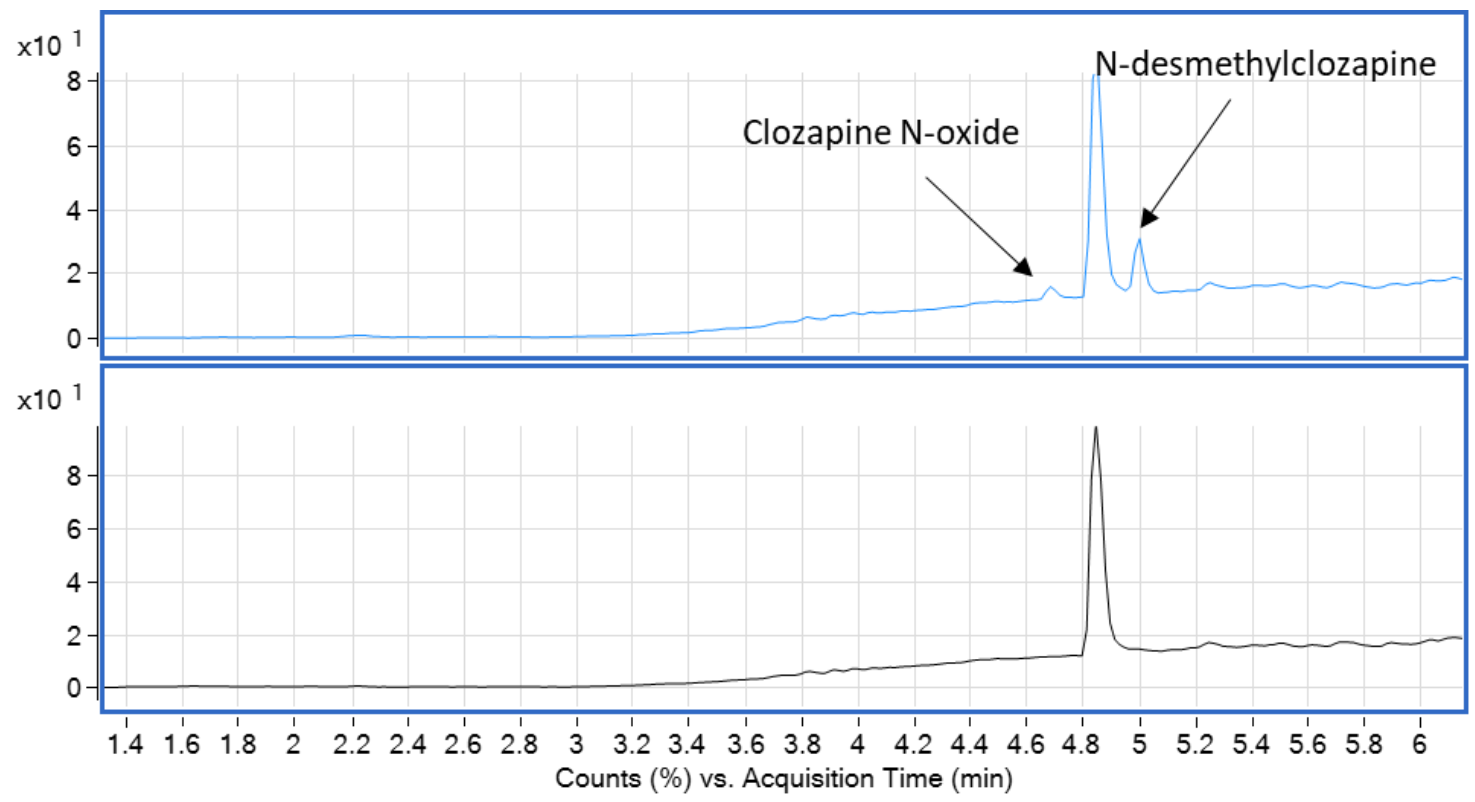

Figure 18. The TICs for the in vitro metabolic assay for clozapine (top) and the negative control with no metabolic system (bottom). The two new peaks are labeled with their corresponding metabolite.

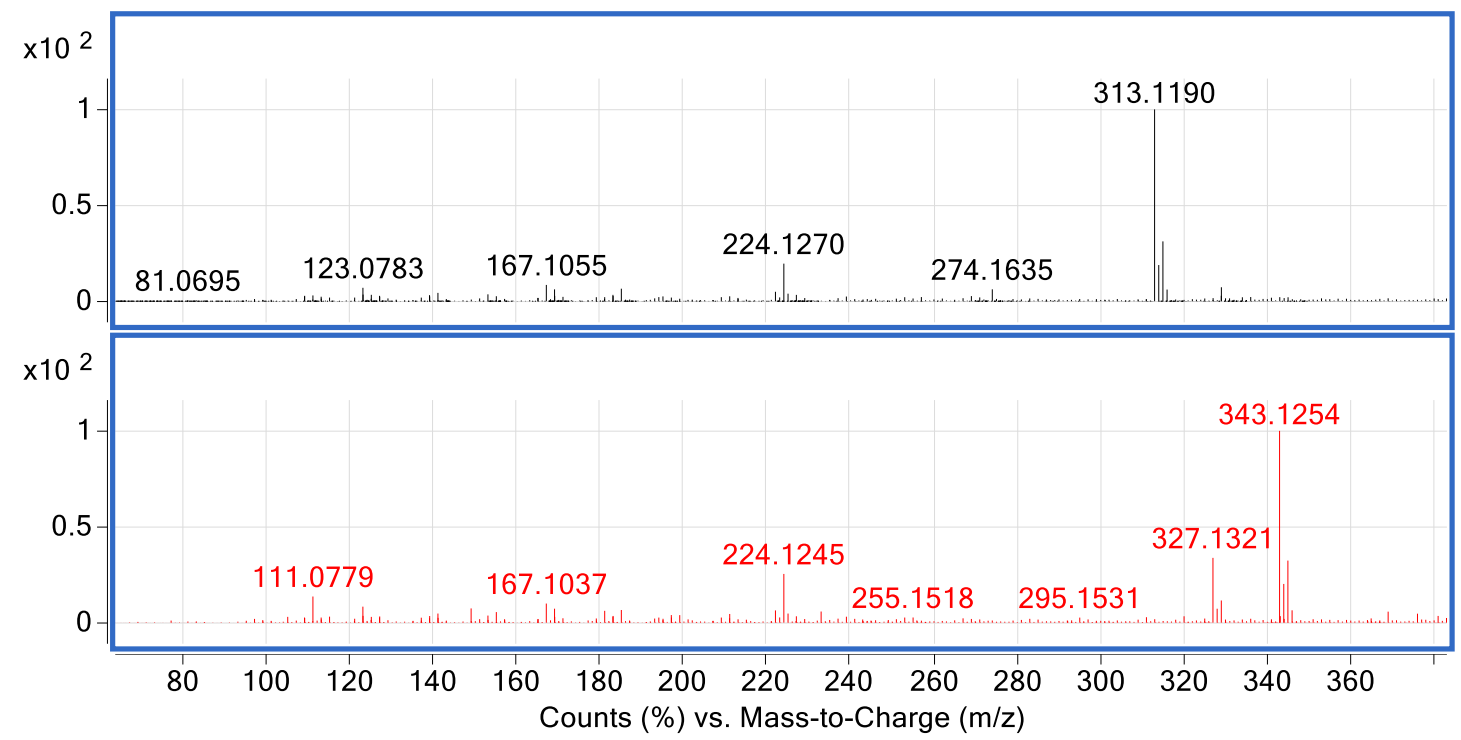

Figure 19. XIC data for N-desmethylclozapine (top) and clozapine $\mathrm{N}$-oxide (bottom).

The stable metabolite data for COC also showed two prominent metabolites, benzoylecgonine and cocaethylene. These data can be seen in Figures 20 and 21. The 
observation of the metabolites for both CLZ and COC implied that the metabolic system utilized would be able to successfully produce the expected metabolites of the drugs of interest.

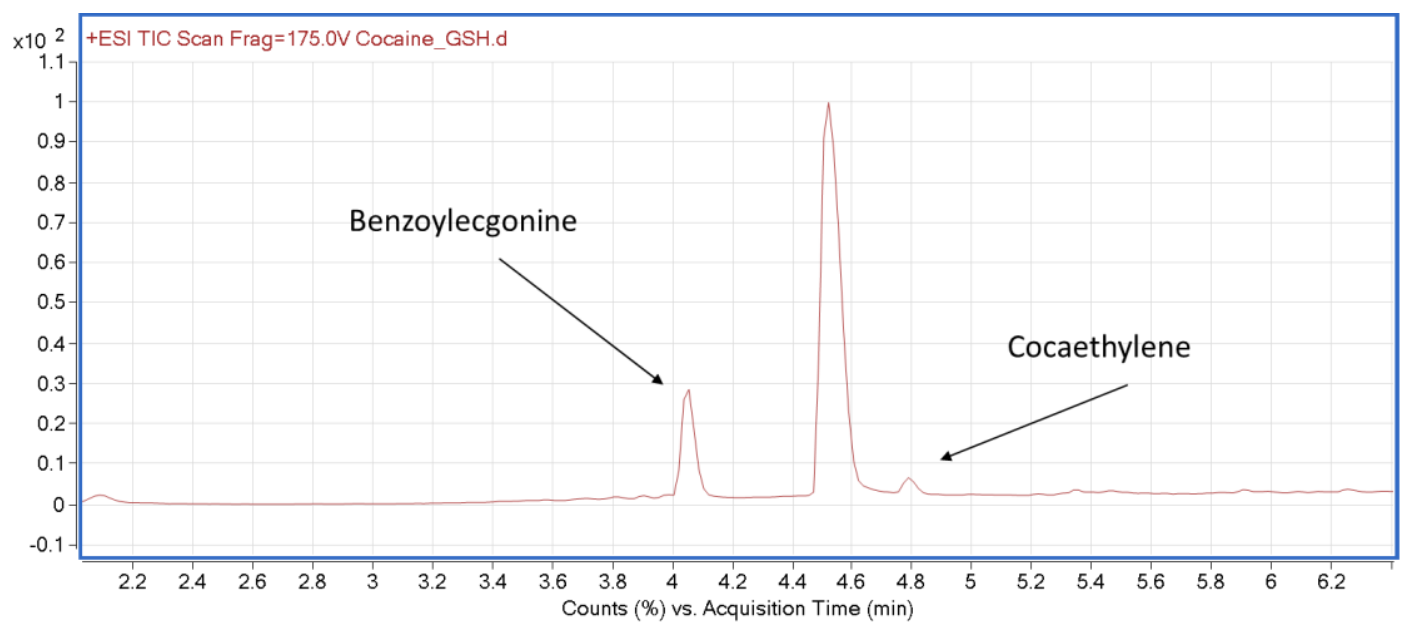

Figure 20 The TICs for the in vitro metabolic assay for COC; the two new peaks are labeled with their corresponding metabolite.

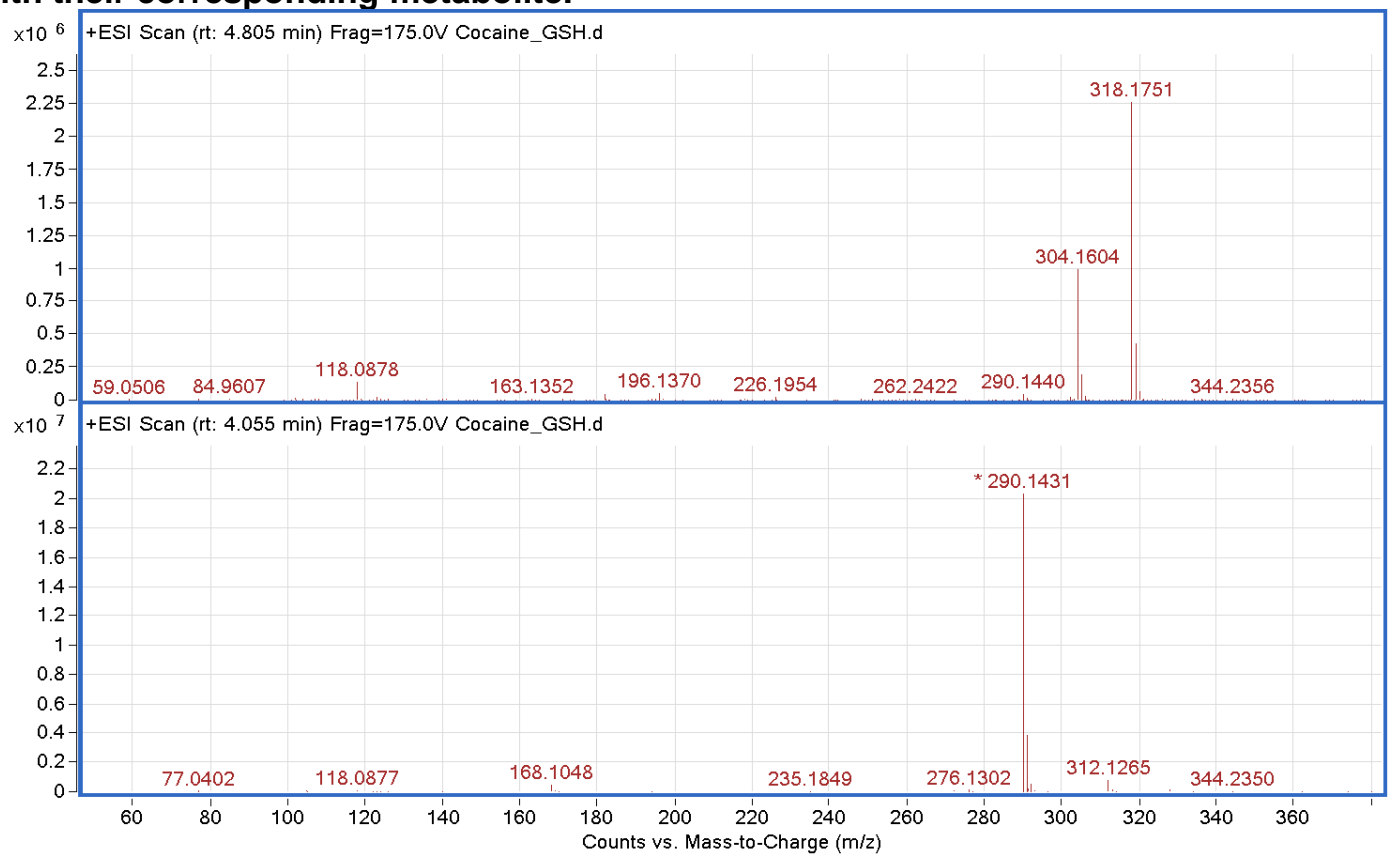

Figure 21 XIC data for cocaethylene (top) and benzoylecgonine (bottom). 


\subsubsection{Task 1 Discussion}

The purpose of this task was to characterize the parent drugs of abuse selected for this study and to assess the effectiveness of the in vitro metabolic assay. To discover how the drugs would behave during the instrumental analysis procedure, retention times were determined for all of the drugs. The positive control acetaminophen is not completely soluble in methanol. This led to acetaminophen being retained on the column and eluting into subsequent sample runs taken that day, as can be seen in Figure 12. Fortunately, this did not interfere with the retention times of any of the other drugs of interest. THC also did not elute at a time similar to the other compounds, and instead was detected towards the end of the run when a high percentage of organic mobile phase was running through the column. This led to no visible peak for THC, a problem which was remedied by the Find by Formula feature of MassHunter's Qualitative software (Figure 14). This may be due to THC being poorly ionized in positive mode, with negative ionization generally being favored for THC analysis. ${ }^{130}$

The fragmentation collected for each drug of interest was obtained in hopes that it would provide insight into the possible fragmentation patterns of any adducted moieties in later studies. All fragmentation data was collected in positive mode, as many of the drugs experience poor ionization using negative mode analysis. While analysis shifted from positive to negative ionization with the GSH adduct studies, the fragmentation collected in Task 1 will be useful in any future GSH research utilizing positive ionization mode analysis of adducts. 
In order to progress to the subsequent tasks of this research, stable metabolite analysis was only preformed using clozapine. While clozapine may have as many as five observed stable metabolites, clozapine $\mathrm{N}$-oxide and $\mathrm{N}$ desmethylclozapine are the most prevalent and were the two metabolites pursued in this task. ${ }^{131}$ These two stable metabolites have been observed in plasma at concentrations approximately the same as the parent drug. ${ }^{132}$ The identification of these two compounds allowed for advancement to the implementation of reactive metabolite assays containing trapping reagents.

\subsection{Task 2: Create and optimize a LC-MS/MS method for the detection and characterization of reactive drug metabolites.}

\subsubsection{Metabolic Trapping Assay Optimization}

Optimization of the in vitro assay system required numerous factors to be examined. Incubation times of $1,3,6$, and $12 \mathrm{~h}$ were examined, with $3 \mathrm{~h}$ determined to be optimal for maximum production of primary metabolites and minimal formation of secondary products. As reported by other investigators ${ }^{84}$, glutathione-S-transferase (GST) was included in several trials to determine if its presence would aid in the formation of adducts with GSH. However, there was no observed increase in adduct formation, and GST was therefore eliminated from the protocol for simplicity. The addition of G6P and G6PD provided a regeneration system for NADPH which allowed for optimal enzymatic activity for the duration of the incubation. Initial trials were conducted with a drug: GSH molar ratio of $1: 20$, however this was later changed to $1: 2$ (final concentration as 
stated in Methods) by increasing the concentration of the drug of interest in order to maximize the concentration of the reactive metabolites formed. Even though this is not an accurate approximation of biologically relevant drug concentrations, it was determined that this amount would provide the greatest opportunity for adduct formation.

\subsubsection{MS and MS/MS Analysis of Glutathione Adducts}

Figure 22a shows an example of a product ion TIC for the positive control drug APAP. A large peak is present at RT $\sim 2.6$ min; the inset shows numerous additional potentially relevant peaks at lower abundances. XIC analysis of APAP-adducted GSH (Figure 22b) revealed one major product at RT 2.68 min with $\mathrm{m} / \mathrm{z} 455$ and a minor product at RT $2.36 \mathrm{~min}$ with $\mathrm{m} / \mathrm{z}$ 471. Bot of the APAPGSH adducts identified in this experiment have been previously reported. ${ }^{80,88}$ The precursor ion scan TIC and XIC data for the remaining drugs can be found in Appendix 1. 

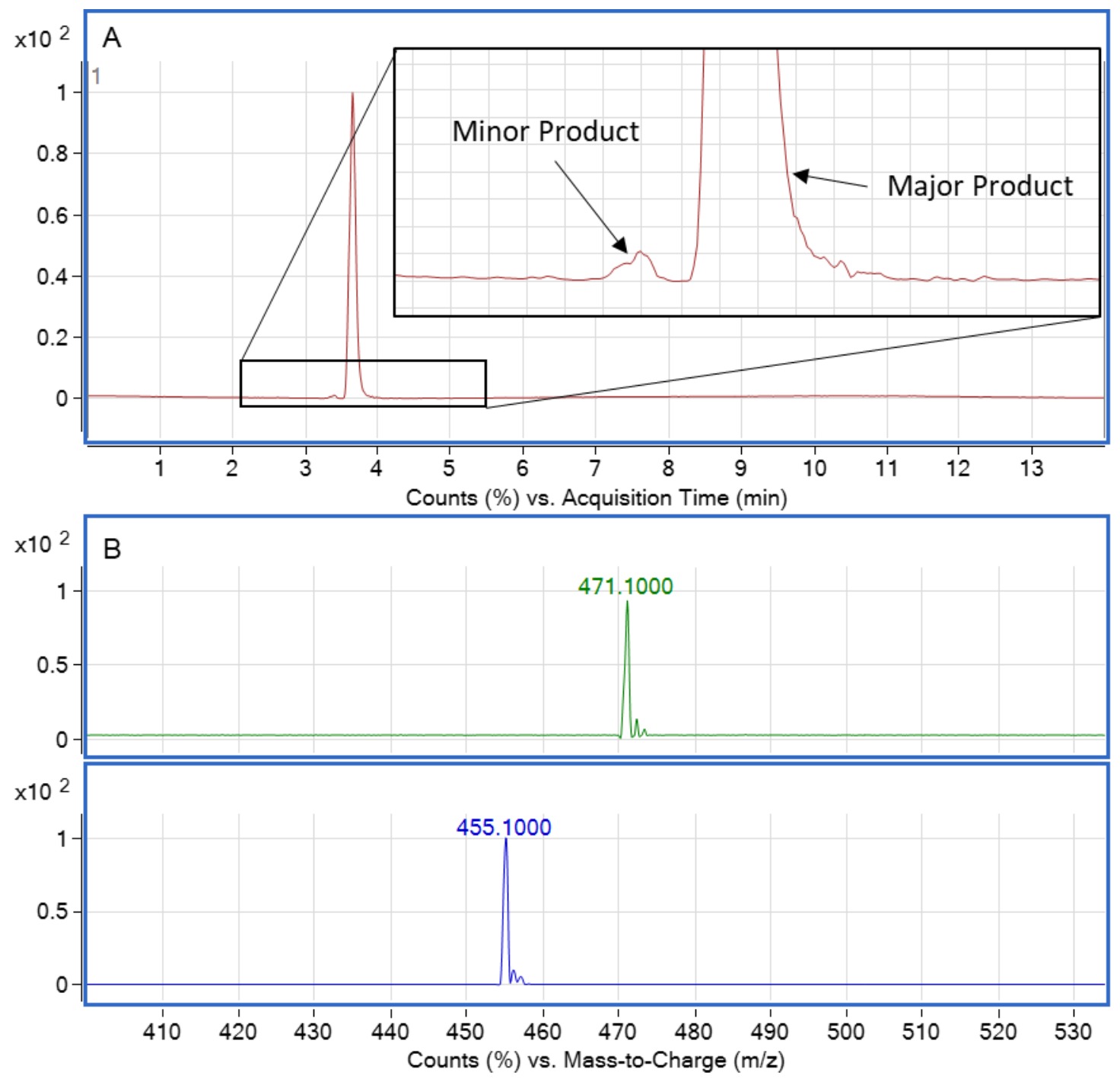

Figure 22. (A) TIC of APAP+GSH collected by product ion scan mode via LC-QqQ-MS in negative ionization mode. Inset is zoomed-in portion around the base of the major peak, showing multiple peaks with lesser intensities. (B) XICs for ions at $m / z 471$ and 455 , the two relevant peaks of interest from the TIC.

Using this approach, a total of 20 potentially significant GSH adduction products were identified by LC-QqQ-MS/MS analysis for 10 of the 16 drugs examined, including APAP, CLZ, COC, DZP, MDMA, MDPV, MOR, NAL, OXY, and THC. Multiple adduct structures were also observed for a number of drugs. In contrast, GSH adducts were not observed for ALP, BUP, DZP, META, METH, 
and METY under the conditions used in the study. Target products detected by low resolution MS were then further examined using high resolution LC-QTOFMS/MS analysis as described below. These potential adducts, and those observed by LC-QTOF-MS/MS analysis, are summarized in Table 8.

\begin{tabular}{|c|c|c|c|}
\hline Drug of Interest & Mass of Drug & $m / z$ via $Q q Q$ & $\mathrm{~m} / \mathrm{z}$ via QTOF \\
\hline \multirow{4}{*}{ APAP } & 151.063 & 435 & 455.089 \\
\hline & & 455 & 471.119 \\
\hline & & 471 & \\
\hline & & 482 & \\
\hline \multirow[t]{4}{*}{ CLZ } & 326.130 & 548 & 548.105 \\
\hline & & 630 & 630.194 \\
\hline & & 648 & 648.498 \\
\hline & & & 652.169 \\
\hline \multirow[t]{2}{*}{ Cocaine } & 303.147 & 468 & 625.230 \\
\hline & & 625 & \\
\hline DZP & 284.072 & 606 & 606.154 \\
\hline \multirow[t]{4}{*}{ MDMA } & 193.110 & 470 & 470.140 \\
\hline & & 481 & 485.189 \\
\hline & & 485 & 498.172 \\
\hline & & 497 & \\
\hline \multirow[t]{3}{*}{ MDPV } & 275.152 & 498 & 498.172 \\
\hline & & 514 & 514.168 \\
\hline & & 567 & 567.238 \\
\hline MOR & 285.136 & 589 & 589.219 \\
\hline \multirow[t]{2}{*}{ NAL } & 341.163 & 645 & 645.249 \\
\hline & & 648 & 663.260 \\
\hline \multirow[t]{3}{*}{ OXY } & 315.147 & 508 & 508.117 \\
\hline & & 619 & 620.019 \\
\hline & & 633 & 633.223 \\
\hline \multirow[t]{4}{*}{ THC } & 314.225 & 650 & 634.306 \\
\hline & & 666 & 650.301 \\
\hline & & & 666.297 \\
\hline & & & 678.334 \\
\hline
\end{tabular}

Table 8. Theoretical adduct masses collected via QQQ-MS/MS and QTOF MS/MS. 
Figure 23 shows the high-resolution mass spectrum of GSH, with corresponding fragment structures indicated. Table 9 presents HRMS data (molecular formula, molecular ion and characteristic fragment ions, and proposed composition) for all GSH adducts detected in the study, along with literature reference if previously reported. The presence and relative abundance of characteristic GSH fragments (i.e., " $b$ ", " $f$ ", " $p$ ", and " $k$ " ions) and fragments associated with drug still bound to GSH (i.e., " $d$ " and "j" ions) provided important data to further elucidate adduct identity and location of the drug-thiol linkage(Figure 24) ${ }^{88}$ In addition, ions associated with fragmentation within the drug moiety itself were occasionally observed. Structural elucidation was facilitated by the generation of fragments at collision energies of 10,20 , and 40 $\mathrm{eV}$ (i.e., top, middle, and bottom panels of each adduct MS/MS spectrum in Figure 25). All MS/MS data for adducts previously reported in the literature are confirmed in Figure 26. 


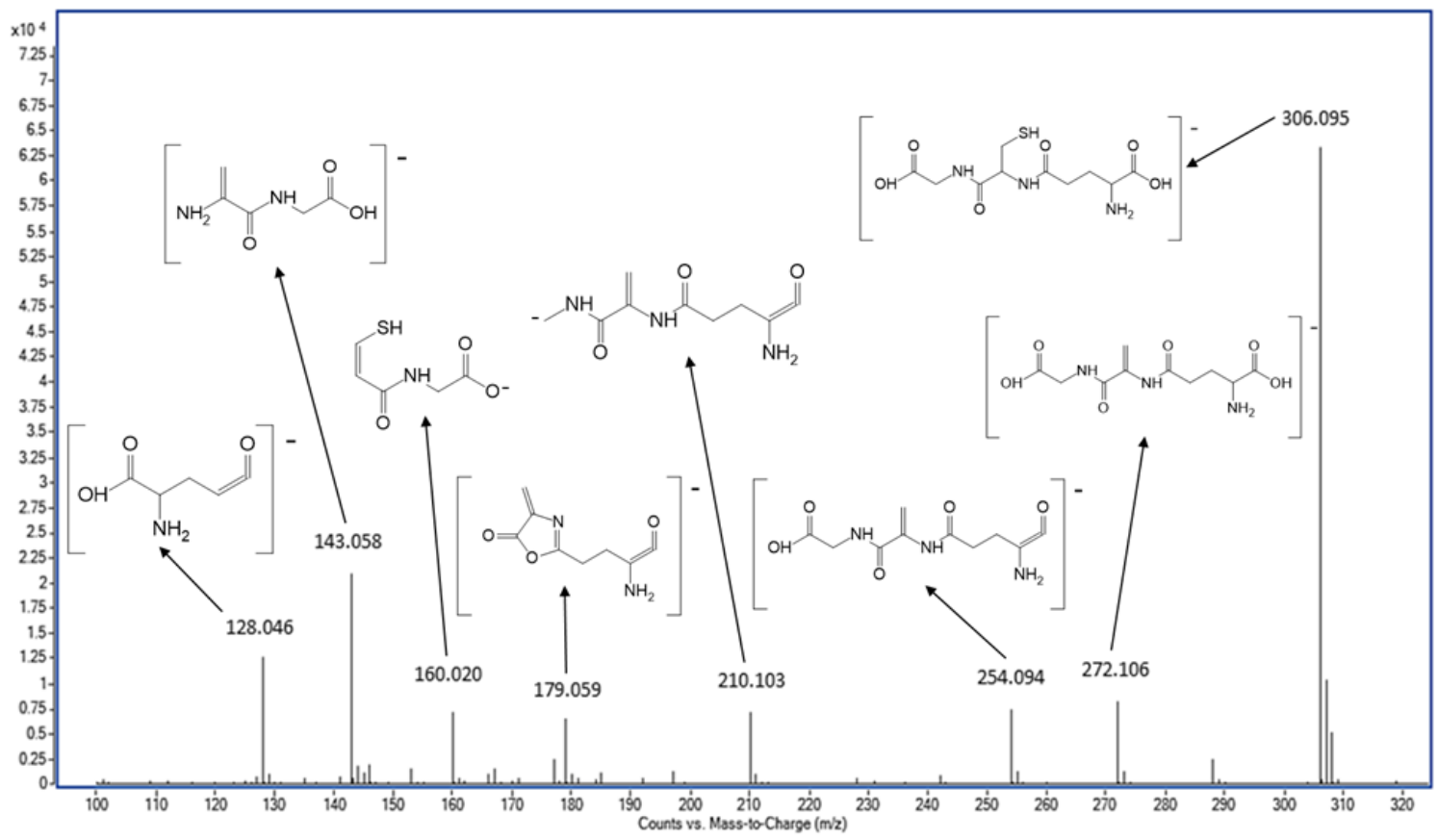

Figure 23. LC-QTOF-MS/MS data collected for glutathione. The peaks labeled all correspond to a known characteristic GSH peak. Negative ionization structures for each peak are provided for each peak.

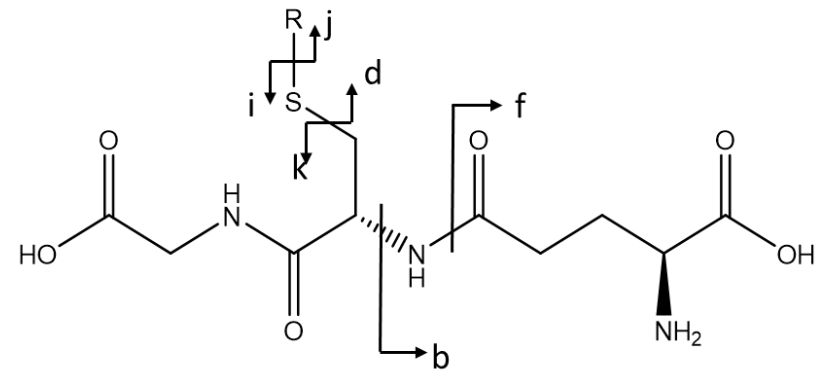

\begin{tabular}{ll}
\hline Fragment & Negative Mode $\mathbf{m} / \mathbf{z}$ \\
\hline $\mathrm{b}$ & 143.0451 \\
$\mathrm{~d}$ & P-273.0961 or 275.1117 \\
$\mathrm{f}$ & 128.0342 \\
i & 306.0765 \\
j & P-307.0838 or 305.0682 \\
k & 272.0883 \\
\hline
\end{tabular}

Figure 24. Detailed breakdown of the nomenclature used for the fragmentation of the GSH adducts. Data presented are from Xie et al. ${ }^{88}$ 

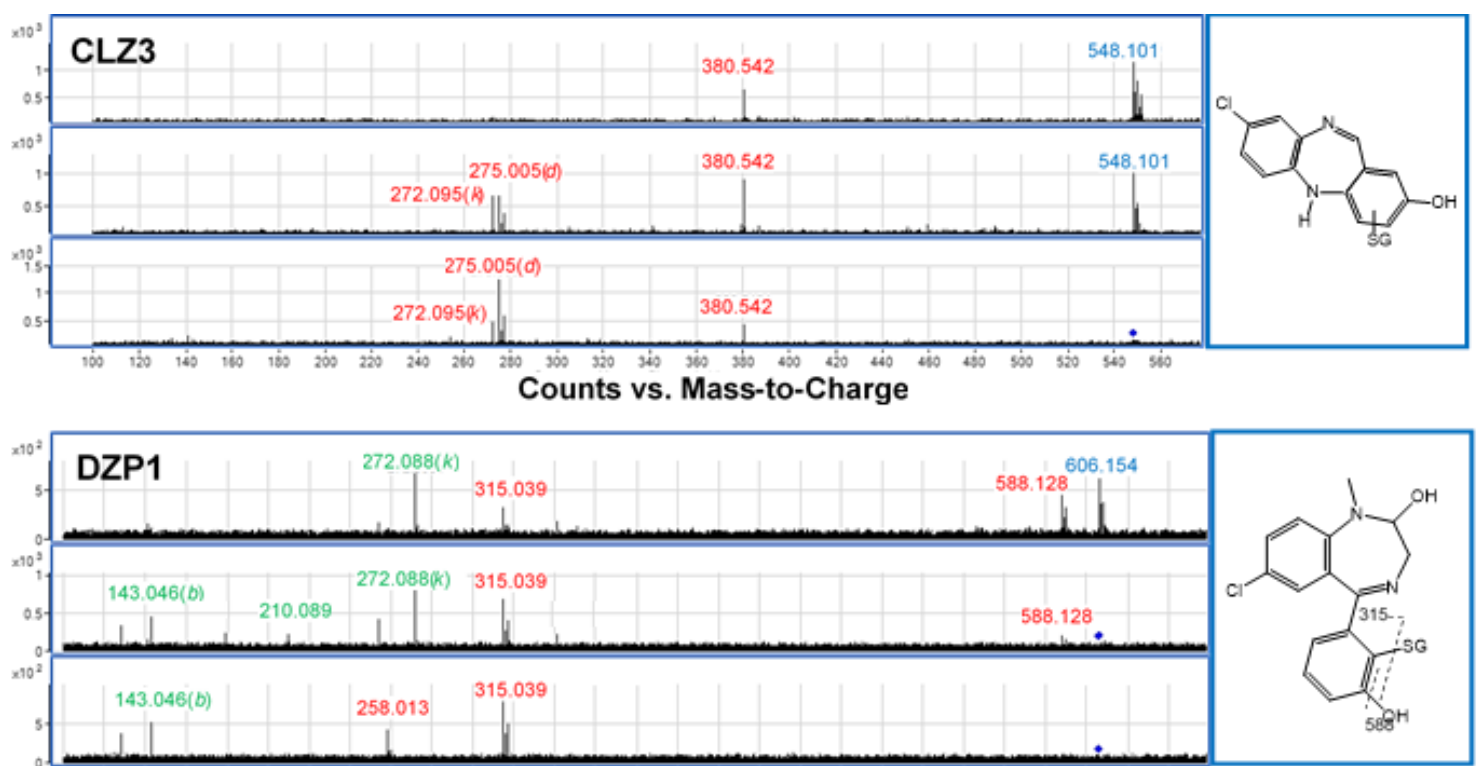

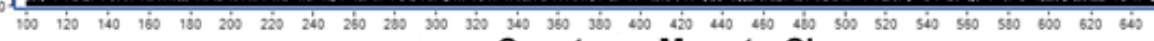
Counts vs. Mass-to-Charge
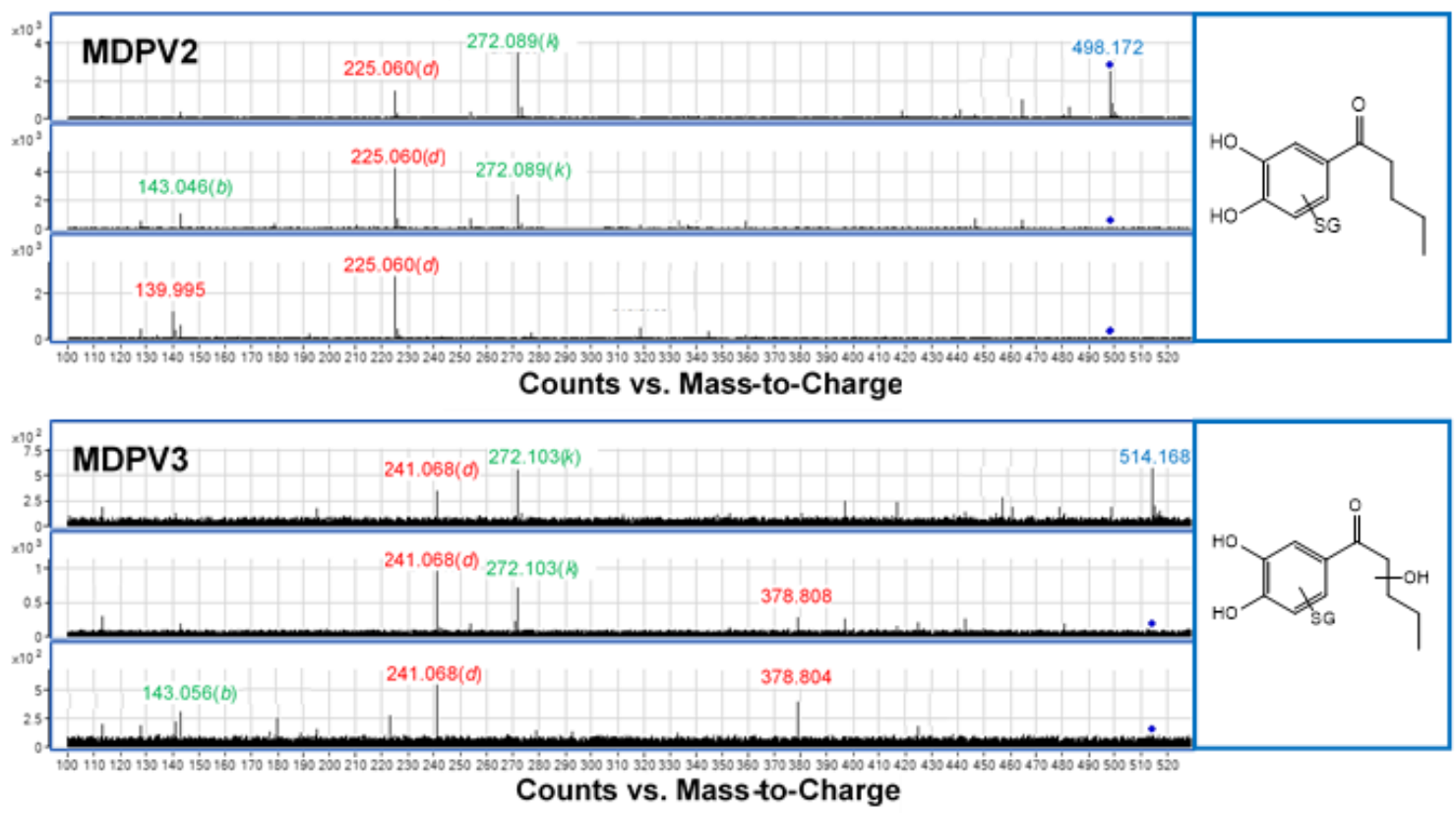

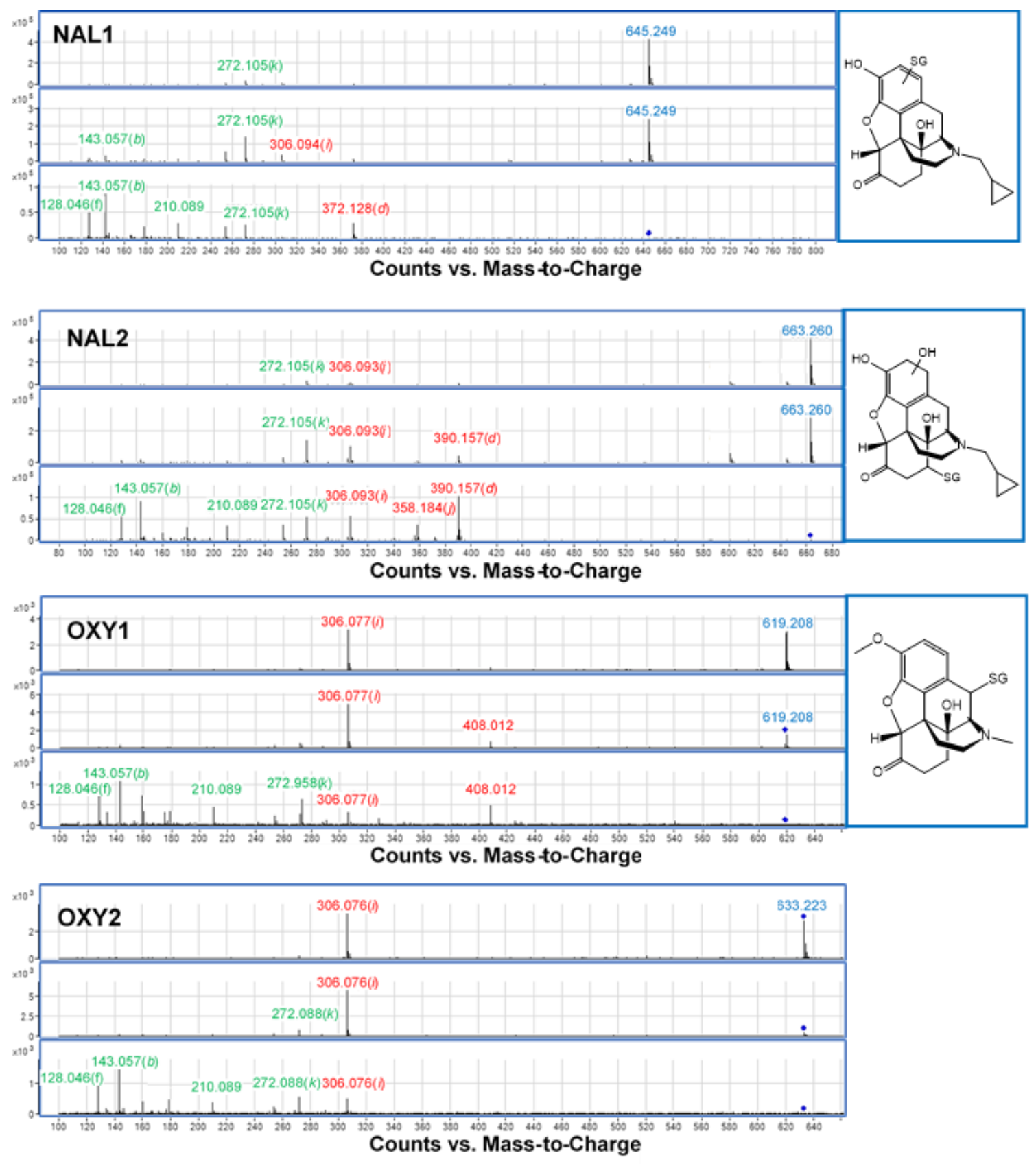

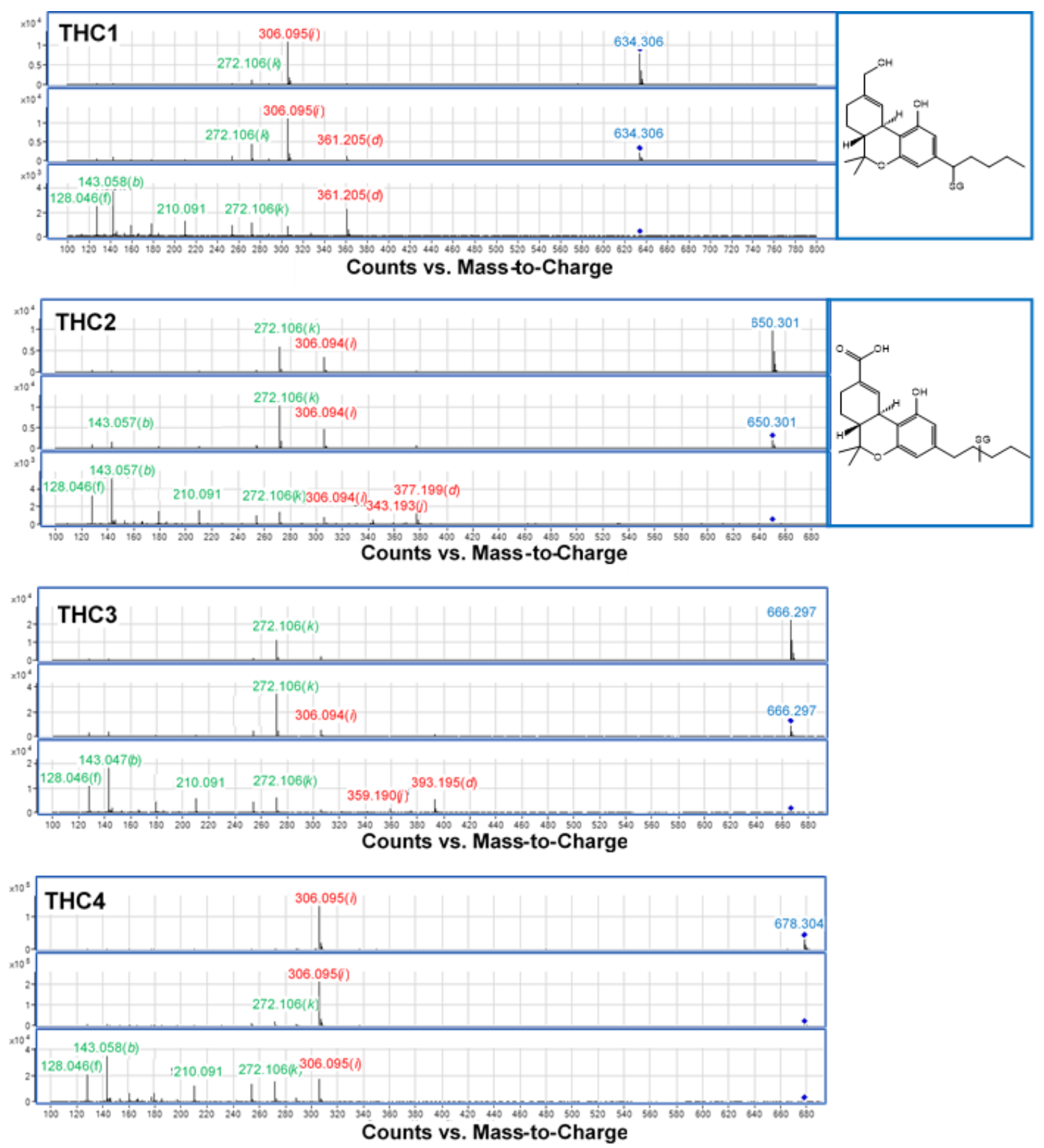

Figure 25. MS/MS spectra for all 12 previously unreported adducts. The molecular ion is represented in blue, GSH-specific peaks in green, and structurally significant peaks for GSH-containing compounds in red. Proposed structures for 9 of the 12 adducts are also shown with GSH linkage indicated. 


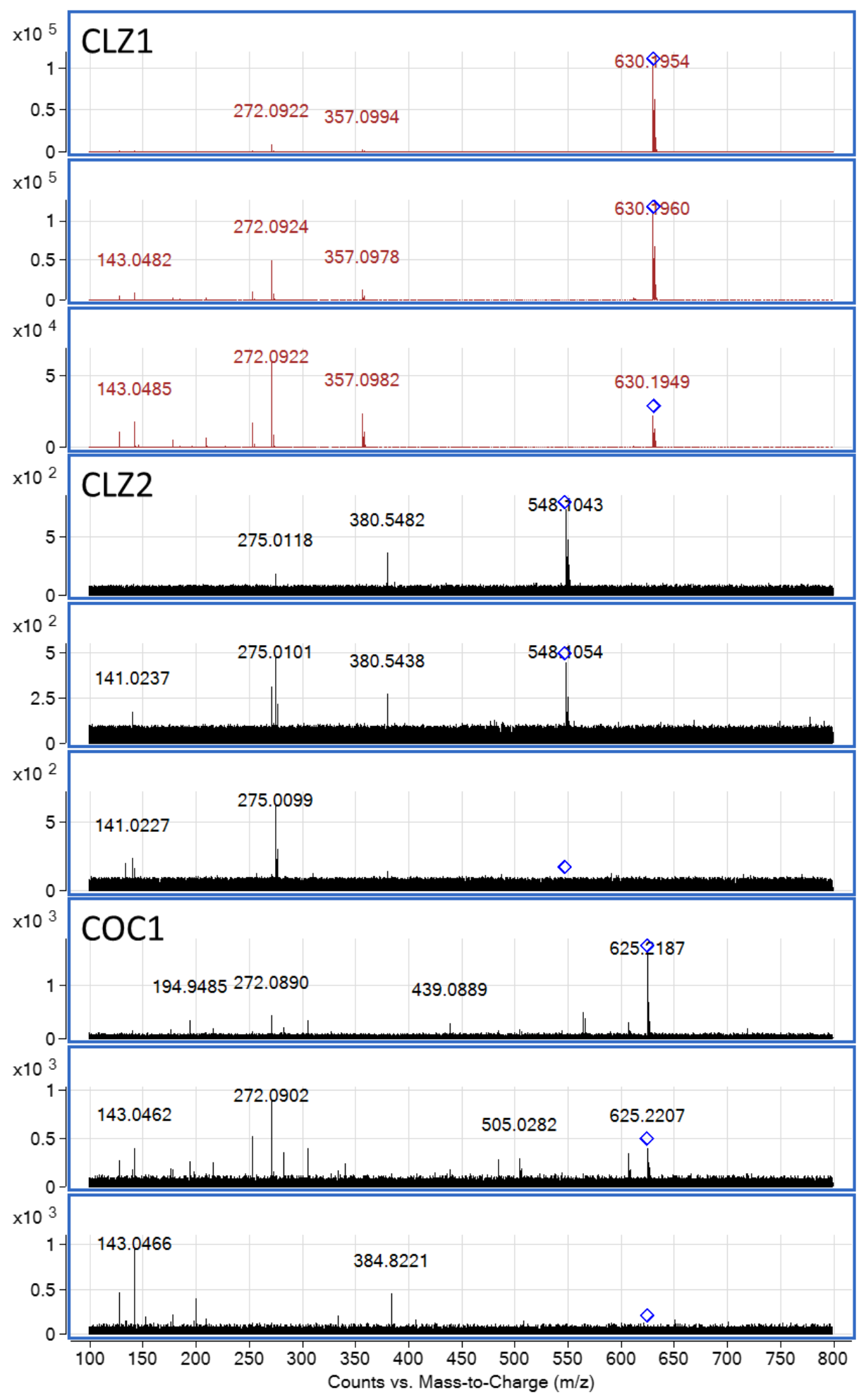




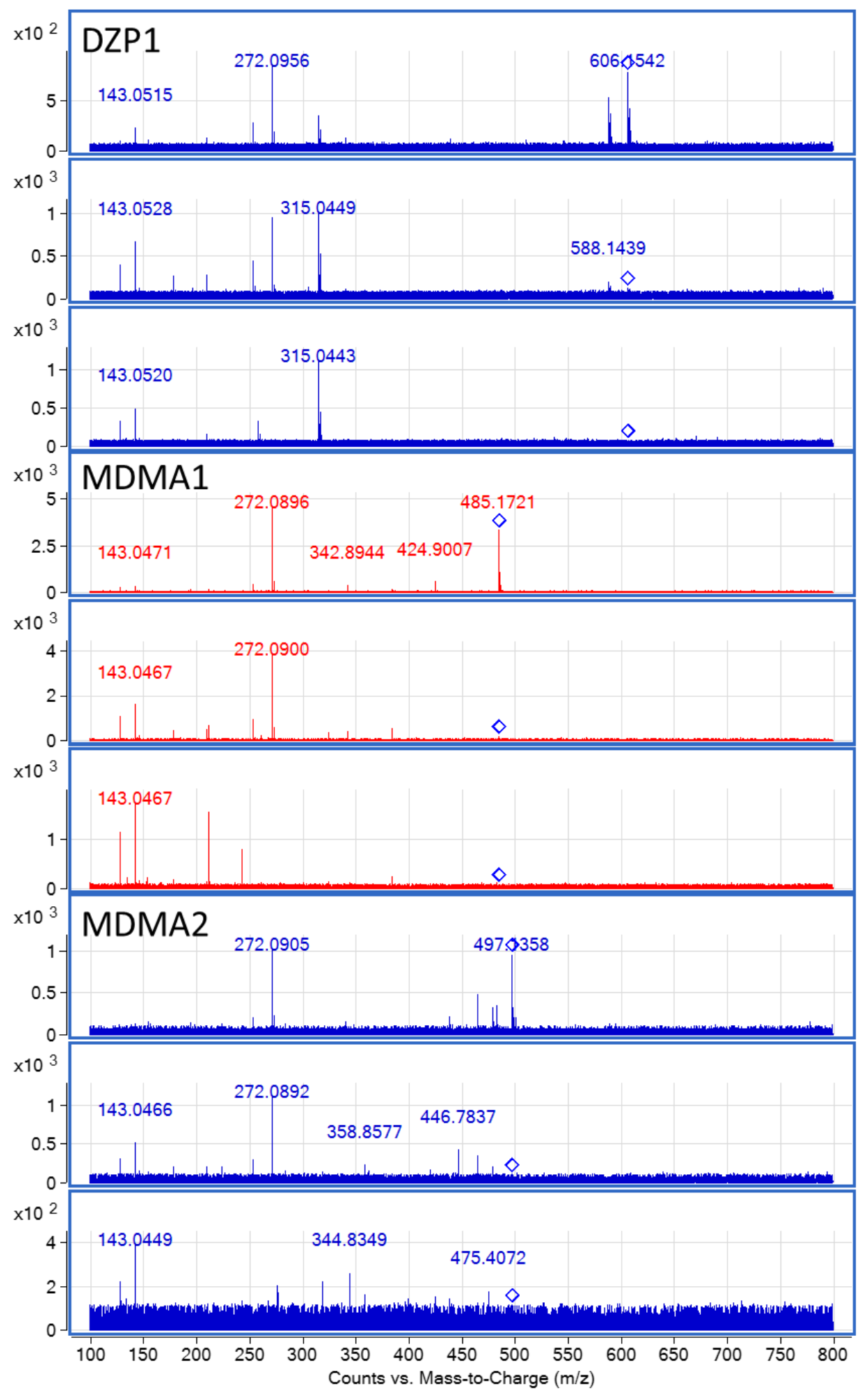




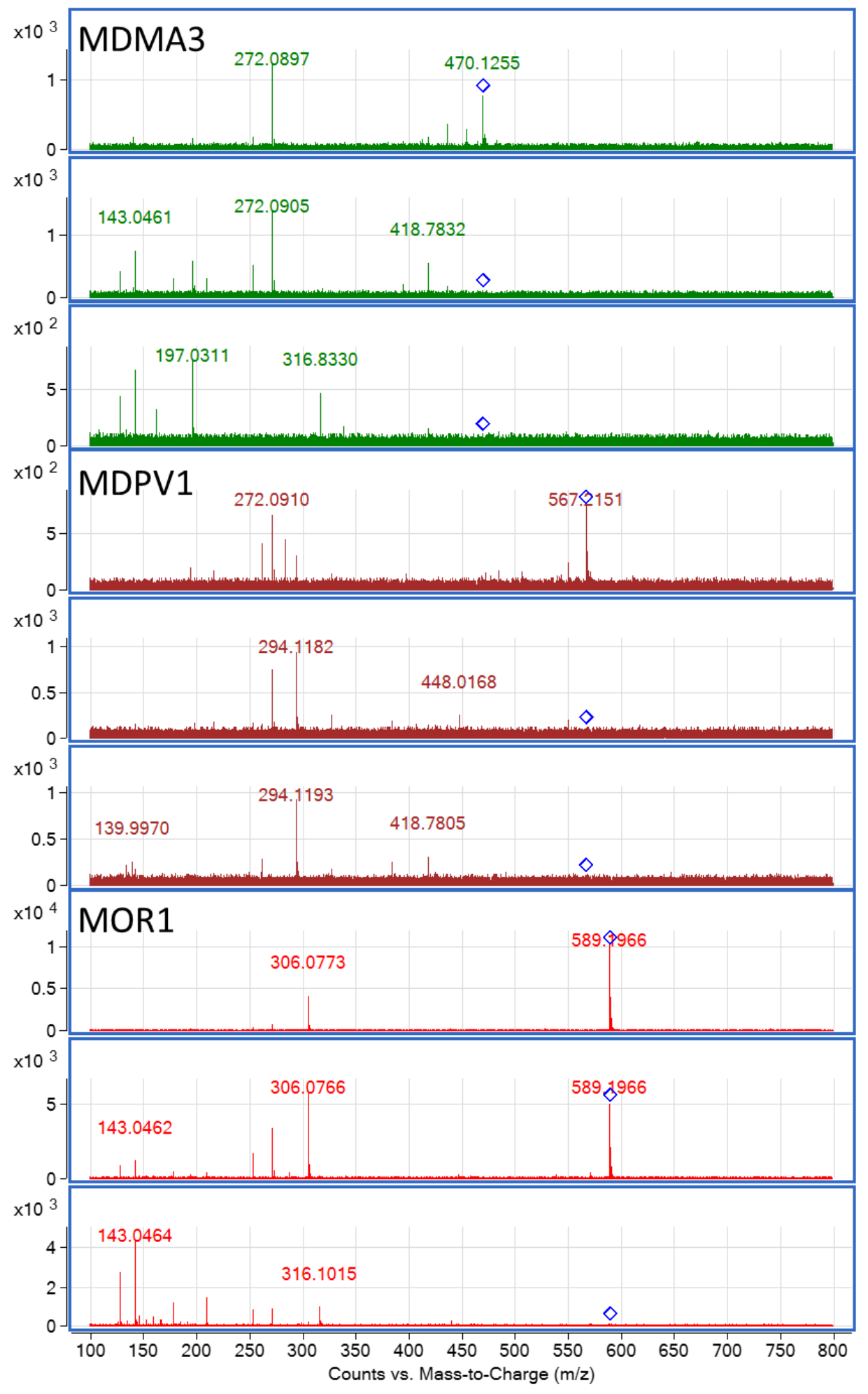

Figure 26. MS/MS data collected for all previously reported GSH adducts 


\begin{tabular}{|c|c|c|c|c|}
\hline Drug & Formula & Composition & Ions Observed & Reference \\
\hline & $\mathrm{C}_{10} \mathrm{H}_{17} \mathrm{~N}_{3} \mathrm{O}_{6} \mathrm{~S}$ & GSH & 306.095, 272.106(k), 254.094, 210.103, 179.059, 160.020, 143.058(b), 128.046(f) & \\
\hline APAP1 & $\mathrm{C}_{18} \mathrm{H}_{24} \mathrm{~N}_{4} \mathrm{O}_{8} \mathrm{~S}$ & $\mathrm{D}+\mathrm{GSH}-2 \mathrm{H}$ & $455.089,272.089(k), 254.078,210.088,182.028(d), 143.045(b), 128.046(f)$ & 74 \\
\hline APAP2 & $\mathrm{C}_{18} \mathrm{H}_{24} \mathrm{~N}_{4} \mathrm{O}_{9} \mathrm{~S}$ & $\mathrm{D}+\mathrm{GSH}+\mathrm{O}-2 \mathrm{H}$ & 471.119, 272.088(k), $198.025(d), 143.045(b), 128.045(f)$ & 82 \\
\hline CLZ1 & $\mathrm{C}_{28} \mathrm{H}_{34} \mathrm{CIN}_{7} \mathrm{O}_{6} \mathrm{~S}$ & $\mathrm{D}+\mathrm{GSH}-2 \mathrm{H}$ & 630.194, 357.095(d), 272.089(k), 254.078, 143.046(b) & 75 \\
\hline CLZ2 & $\mathrm{C}_{28} \mathrm{H}_{36} \mathrm{CIN}_{7} \mathrm{O}_{7} \mathrm{~S}$ & $\mathrm{D}+\mathrm{GSH}+\mathrm{O}$ & 648.198, 272.089(k), 143.046(b) & 75 \\
\hline CLZ3 & $\mathrm{C}_{23} \mathrm{H}_{24} \mathrm{CIN}_{5} \mathrm{O}_{7} \mathrm{~S}$ & $\mathrm{D}+\mathrm{GSH}+\mathrm{O}-\mathrm{C}_{5} \mathrm{H}_{10} \mathrm{~N}_{2}$ & 548.101, 275.005(d), 272.095(k), 143.046(b) & None \\
\hline COC1 & $\mathrm{C}_{27} \mathrm{H}_{38} \mathrm{~N}_{4} \mathrm{O}_{11} \mathrm{~S}$ & $\mathrm{D}+\mathrm{GSH}+\mathrm{O}$ & 625.230, 565.055, 384.821, 306.080(I), 272.090(k), 194.950, 143.046(b) & 57 \\
\hline DZP1 & $\mathrm{C}_{26} \mathrm{H}_{30} \mathrm{CIN}_{5} \mathrm{O}_{8} \mathrm{~S}$ & $\mathrm{D}+\mathrm{GSH}+\mathrm{O}$ & 606.154, 588.142, 315.039, 272.088(k), 258.013, 210.089, 143.046(b) & None \\
\hline MDMA1 & $\mathrm{C}_{20} \mathrm{H}_{30} \mathrm{~N}_{4} \mathrm{O}_{8} \mathrm{~S}$ & $\mathrm{D}+\mathrm{GSH}-\mathrm{CH}_{2}$ & 485.189, 272.090(k), 212.771(d), 143.047(b), 128.045(f) & 78 \\
\hline MDMA2 & $\mathrm{C}_{21} \mathrm{H}_{30} \mathrm{~N}_{4} \mathrm{O}_{8} \mathrm{~S}$ & $\mathrm{D}+\mathrm{GSH}-2 \mathrm{H}$ & 497.131, 272.091(k), 254.085, 143.047(b), 128.046(f) & 78 \\
\hline MDMA3 & $\mathrm{C}_{19} \mathrm{H}_{25} \mathrm{~N}_{3} \mathrm{O}_{9} \mathrm{~S}$ & $\mathrm{D}+\mathrm{GSH}+\mathrm{O}-\mathrm{C}_{2} \mathrm{H}_{7} \mathrm{~N}$ & 470.140, 436.793, 272.090(k), 197.030(d), 143.046(b), 128.045(f) & 78 \\
\hline MDPV1 & $\mathrm{C}_{25} \mathrm{H}_{36} \mathrm{~N}_{4} \mathrm{O}_{9} \mathrm{~S}$ & $\mathrm{D}+\mathrm{GSH}-\mathrm{CH}_{2}$ & $567.238,294.118(d), 272.091(k)$ & 78 \\
\hline MDPV2 & $\mathrm{C}_{21} \mathrm{H}_{29} \mathrm{~N}_{3} \mathrm{O}_{9} \mathrm{~S}$ & $\mathrm{D}+\mathrm{GSH}-\mathrm{C}_{5} \mathrm{H}_{8} \mathrm{~N}$ & 498.172, 272.089(k), 225.060(d), 143.046(b), 139.995 & None \\
\hline MDPV3 & $\mathrm{C}_{21} \mathrm{H}_{29} \mathrm{~N}_{3} \mathrm{O}_{10} \mathrm{~S}$ & $\mathrm{D}+\mathrm{GSH}+\mathrm{O}-\mathrm{C}_{5} \mathrm{H}_{7} \mathrm{~N}$ & 514.168, 378.808, 272.103(k), 241.068(d), 143.056(b) & None \\
\hline MOR1 & $\mathrm{C}_{27} \mathrm{H}_{34} \mathrm{~N}_{4} \mathrm{O}_{9} \mathrm{~S}$ & $\mathrm{D}+\mathrm{GSH}-\mathrm{OH}$ & 589.219, 316.102(d), 306.077(I), 272.089(k), 210.089, 143.046(b), 128.045(f) & 54 \\
\hline NAL1 & $\mathrm{C}_{30} \mathrm{H}_{38} \mathrm{~N}_{4} \mathrm{O}_{10} \mathrm{~S}$ & $\mathrm{D}+\mathrm{GSH}-2 \mathrm{H}$ & 645.249, 306.094(I), 272.105(k), 210.089, 143.057(b), 128.046(f) & None \\
\hline NAL2 & $\mathrm{C}_{30} \mathrm{H}_{40} \mathrm{~N}_{4} \mathrm{O}_{11} \mathrm{~S}$ & $\mathrm{D}+\mathrm{GSH}+\mathrm{O}$ & $\begin{array}{l}\text { 663.260, 390.157(d), 358.184(J), 306.093(l), 272.105(k), 210.089, 143.057(b), } \\
128.046(f)\end{array}$ & None \\
\hline OXY1 & $\mathrm{C}_{28} \mathrm{H}_{36} \mathrm{~N}_{4} \mathrm{O}_{10} \mathrm{~S}$ & $\mathrm{D}+\mathrm{GSH}-2 \mathrm{H}$ & 619.208, 408.012, 306.077(I), 272.958(k), 210.089, 143.057(b), 128.046(f) & None \\
\hline
\end{tabular}




\begin{tabular}{|c|c|c|c|c|}
\hline OXY2 & $\mathrm{C}_{28} \mathrm{H}_{34} \mathrm{~N}_{4} \mathrm{O}_{11} \mathrm{~S}$ & $\mathrm{D}+\mathrm{GSH}+\mathrm{O}-4 \mathrm{H}$ & 633.223, 306.076(l), 272.088(k), 210.089, 143.057(b), 128.046(f) & None \\
\hline $\mathrm{THC1}$ & $\mathrm{C}_{31} \mathrm{H}_{45} \mathrm{~N}_{3} \mathrm{O}_{9} \mathrm{~S}$ & $\mathrm{D}+\mathrm{GSH}+\mathrm{O}-2 \mathrm{H}$ & 634.306, 361.205(d), 306.095(I), 272.106(k), 210.091, 143.058(b), 128.046(f) & None \\
\hline THC2 & $\mathrm{C}_{31} \mathrm{H}_{45} \mathrm{~N}_{3} \mathrm{O}_{10} \mathrm{~S}$ & $\mathrm{D}+\mathrm{GSH}+\mathrm{O}_{2}-2 \mathrm{H}$ & $\begin{array}{l}\text { 650.301, 377.199(d), 343.193(j), 306.094(l), 272.106(k), 210.091, 143.057(b), } \\
128.046(f)\end{array}$ & None \\
\hline THC3 & $\mathrm{C}_{31} \mathrm{H}_{45} \mathrm{~N}_{3} \mathrm{O}_{11} \mathrm{~S}$ & $\mathrm{D}+\mathrm{GSH}+\mathrm{O}_{3}-\mathrm{H}$ & $\begin{array}{l}\text { 666.297, 393.195(d), 359.190(j), 306.094(l), 272.106(k), 210.091, 143.057(b), } \\
128.046(f)\end{array}$ & None \\
\hline THC4 & $\mathrm{C}_{31} \mathrm{H}_{41} \mathrm{~N}_{3} \mathrm{O}_{12} \mathrm{~S}$ & $\mathrm{D}+\mathrm{GSH}+\mathrm{O}_{4}-4 \mathrm{H}$ & 678.304, 306.095(I), 272.106(k), 210.091, 143.058(b), 128.046(f) & None \\
\hline
\end{tabular}

aMolecular ion in bold; letters in parentheses refer to characteristic GSH fragments according to nomenclature of Xie et al. (2013).

${ }^{b}$ Literature reference for previously reported adduct; none - not previously reported.

Table 9. Summary of fragmentation of all GSH adducts observed. 


\subsubsection{Glutathione Adduct Structure Identification}

As an example of the procedure used to confirm GSH adduct formation and identity based on HRMS data, the molecular ion peak observed at $\mathrm{m} / \mathrm{z}$ 455.089 for APAP was consistent with a previously reported adduct of the parent drug with GSH (APAP1; Figure 27a and Table 9) Major fragment ions for this adduct included the molecular ion ( $\mathrm{m} / \mathrm{z} 455.089)$, five fragments specific to $\mathrm{GSH}$ $(m / z 272.089,254.078,210.088,143.045,128.046)^{88}$, and a fragment corresponding to the drug moiety bound to the sulfur of GSH $(\mathrm{m} / \mathrm{z} 182.028)$. A second, less intense molecular ion peak for an acetaminophen-GSH adduct (APAP2) was observed at $m / z 471.119$, along with three fragments consistent with GSH modification ( $m / 2272.088,143.045$, and 128.045$)$ in addition to a fragment corresponding to the drug moiety bound to the sulfur of GSH $(\mathrm{m} / \mathrm{z}$ 198.025) (Figure 27b and Table 9). To determine potential structures for this adduct, the parent drug was subjected to manual metabolic modification analysis using ChemDraw Prime software (PerkinElmer, version 16.0) to produce a list of likely alterations occurring from Phase I metabolism. This analysis indicated that hydroxylation of the phenyl ring would produce calculated molecular ion and fragment masses consistent with those observed. This proposed most likely adduct structure has also been previously reported. ${ }^{88}$ 


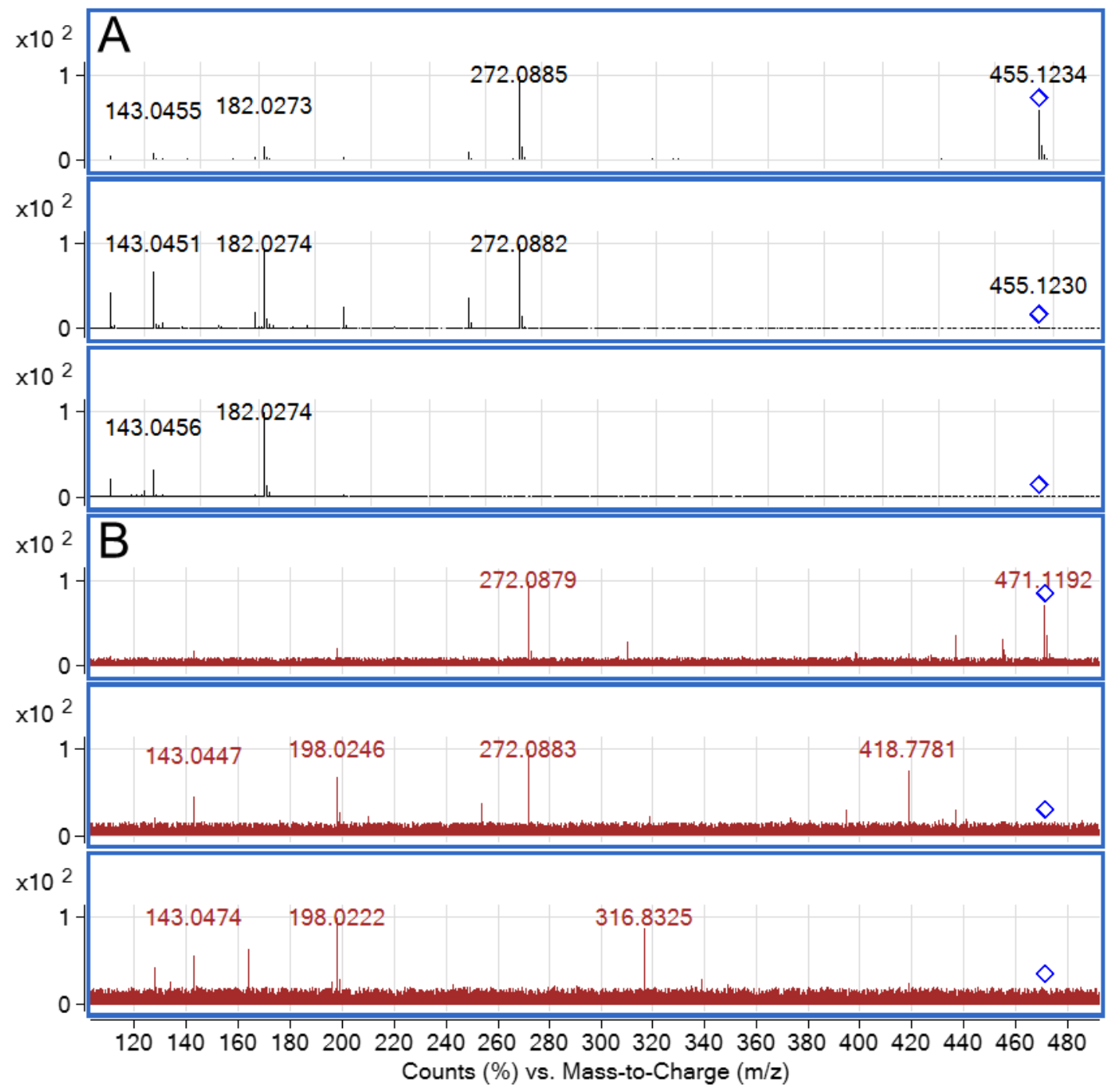

Figure 27. MS/MS data collected for APAP1 and APAP2.

The same general procedure was repeated for all detected putative drugGSH adducts. Figure 25 shows QTOF MS/MS spectra and proposed structures for all GSH adducts identified in the present study that have not been previously reported in the literature. For clozapine, three GSH adducts were detected. Data for two of these (CLZ1 and CLZ2) are consistent with adducts previously reported by other investigators. In contrast, CLZ3 (Figure 25) represents a novel 
GSH adduct structure for this drug. For CLZ3, the $[\mathrm{M}-\mathrm{H}]^{-}$ion with $\mathrm{m} / \mathrm{z}$ of 548.099 is consistent with loss of the entire piperazine moiety, in addition to hydroxylation and rearomatization following adduction with GSH. The peak labeled as a " $d$ " transition at $m / z 275.005$ corresponds to the neutral loss observed from the cleavage of the sulfur of GSH from the rest of the tripeptide. The resulting fragment represents the metabolized CLZ moiety still bound to the sulfur of GSH. The " $k$ " ion corresponds to a characteristic GSH fragment. Rearomatization of the benzene ring is supported by the presence of the " $k$ " peak and lack of an " $i$ " peak, suggesting that conjugation occurs at an aromatic site on the clozapine metabolite. ${ }^{88}$

DZP1 is a previously unreported adduct for diazepam and was the only one observed for this drug. The presence of the $[\mathrm{M}-\mathrm{H}]^{-}$ion at $\mathrm{m} / \mathrm{z} 606.154$ is consistent with a mechanism involving reduction of the diazepine ketone in the parent drug to a hydroxyl group, hydroxylation of the 5-phenyl ring, and covalent adduction of GSH with rearomatization of the phenyl group. The specific fragment at $m / z 588.142$ is consistent with cleavage of a hydroxyl group, most likely that attached to the 5-phenyl moiety. The specific fragment at $m / z 315.044$ may be derived via cleavage of both the 5-phenyl hydroxyl and the phenyl C-S bond of the adduct. Rearomatization of the phenyl ring containing the site of adduction is supported by the presence of the " $k$ " fragment at $m / z 272.098$ in addition to the lack of an " $"$ " fragment. The remaining " $b$ " and $m / z 210.093$ ions are each consistent with GSH fragmentation. 
MDPV1 is identical in MS/MS characteristics to a previously reported GSH adduct with this compound and which represents adduction to a demethylenated diol metabolite. ${ }^{84}$ MDPV2 is the first of two previously unreported MDPV adducts observed in this study. The [M-H]' ion at $m / z 498.172$ most likely represents metabolic demethylenation, as with MDPV1, in addition to further metabolic loss of the pyrrolidine ring via $\mathrm{N}$-deamination, followed by covalent adduction by the GSH thiol on the phenolic ring. The presence of a " $d$ " fragment at $m / z 225.060$ further supports the identity of the adducted drug moiety, as it corresponds to the mass of the moiety plus the sulfur of GSH. The presence of a " $k$ " fragment with $\mathrm{m} / \mathrm{z}$ 272. 089 and lack of a detectable " $"$ " fragment strongly indicates linkage of the GSH sulfur to an aromatic carbon. The remaining " $b$ " ion at $m / z 143.046$ represents a GSH fragment.

MDPV3 is a second unreported GSH adduct for this compound. Similar to MDPV2, the $[\mathrm{M}-\mathrm{H}]^{-}$ion at $\mathrm{m} / \mathrm{z} 514.168$ is consistent with demethylenation, loss of the pyrrolidine ring, and covalent adduction of the GSH thiol. However, the precursor ion mass for MDPV3 also indicates addition of a hydroxyl group, most likely on the alkyl chain moiety. This interpretation is corroborated by the presence of a " $d$ " ion with $m / z 241.068$. Again, the presence of a " $k$ " fragment at $m / z 272.103$ along with the lack of an " $\rho$ " fragment supports an aromatic thiol linkage. The remaining " $b$ " ion represents a GSH fragment, while the ion at $m / z$ 378.808 is unidentified.

Two naltrexone-GSH adducts were observed in the present study and are the first such derivatives reported in the literature. For NAL1, a $[\mathrm{M}-\mathrm{H}]^{-}$ion at $\mathrm{m} / \mathrm{z}$ 
645.249 is consistent with unmodified precursor drug directly adducted to GSH. The MS/MS spectrum clearly shows the presence of " $k$ " ions with lower intensities of " $r$ " and " $d$ " ions (Figure 25). While not unequivocal, this pattern is most consistent with thiol conjugation to the benzene ring in naltrexone. The " $f$ " and " $b$ " ions represent GSH fragments.

The MS/MS spectrum for NAL2 exhibits an $[\mathrm{M}-\mathrm{H}]^{-}$ion at $\mathrm{m} / \mathrm{z} 663.260$, which is most consistent with GSH conjugation to a hydroxylated metabolite. This interpretation is also supported by the presence of a " $d$ " fragment at $m / z$ 390.157 and a " $"$ " fragment at $m / z$ 358.184. The similar relative abundances of the " $k$ " and " $l$ " ions along with the higher abundance of " $d$ " as compared to " $j$ " ions suggest linkage of the thiol sulfur of GSH to an aliphatic carbon in the drug moiety. ${ }^{88}$ While Figure 25 shows one possible structure consistent with these data, the position of the hydroxyl group and C-S link cannot be unequivocally determined without additional information.

GSH adducts with oxycodone have not previously been reported. In the present study, OXY1 exhibited a [M-H] ion at $m / z 619.208$, consistent with $\mathrm{GSH}$ adduction to parent drug. The presence of an "i" fragment at $m / z 306.077$ at a much higher abundance than the " $k$ " ion at $m / z 272.958$, along with the absence of a " $d$ " ion, is strongly suggestive of a GSH thiol linkage to a benzylic carbon. This determination of a benzylic linkage is in agreeance with the literature on GSH adduct linkage sites. ${ }^{88}$ Based on these data, a possible structure for the OXY1 adduct is shown in Figure 25. Evidence for a second GSH-oxycodone adduct (OXY2) was also obtained. The $[\mathrm{M}-\mathrm{H}]^{-}$ion at $m / z 633.223$ is $14 \mathrm{Da}$ larger 
than the OXY1 species, suggesting GSH adduction to a metabolite of the drug. As with OXY1, the presence of a high abundance " $i$ " fragment at $\mathrm{m} / z 306.077$ combined with the lack of a " $d$ " ion is strongly suggestive of a GSH thiol linkage to a benzylic carbon. However, without additional data, a putative structure for this adduct is not proposed.

GSH adducts of $\Delta^{9}$-tetrahydrocannabinol (THC) have not previously been reported in the literature. The present study identified four species consistent with covalent adduction of GSH with THC, all proposed to result from modification of THC metabolites oxidized at the 11 position or on the pentyl chain of the parent drug. THC1 exhibited a [M-H] ion at $m / z 634.306$, consistent with GSH adduction to 11 -hydroxy- $\Delta^{9}-\mathrm{THC}$, a prominent metabolite of this cannabinoid. ${ }^{88}$ The presence of a major " $d$ " ion at $m / z 361.205$ in addition to the " $i$ " fragment further supports this interpretation. The data most support a benzylic C-S linkage between the drug moiety and GSH, based on the substantially higher intensity of the " $"$ " fragment as compared to that of the " $k$ " ion (Figure 25). However, due to the presence of a definite " $d$ " fragment, an aliphatic linkage cannot be entirely ruled out.

The THC2 moiety exhibited a [M-H] ion at $m / z 650.301$, which is consistent with conjugation of GSH with another common THC metabolite, i.e., 11-nor-9-carboxy- $\Delta^{9}$-THC. This interpretation is also supported by the presence of a " $d$ " fragment at $m / z 377.199$. The covalent linkage between the drug moiety and the GSH sulfur is most likely aliphatic, since the intensity of the " $k$ " ion (at $m / z 272.106$ ) is higher than that of the " $i$ " fragment at $m / z 306.094$. 
The structures of the detected adducts represented by THC3 and THC4 are more speculative, as they appear to involve adduction of secondary oxidized THC metabolites. For example, THC3 exhibited a [M-H]- ion at $m / z 666.297$, which would be consistent with GSH adduction to a hydroxylated metabolite of 11-nor-9-carboxy- $\Delta^{9}-\mathrm{THC}$, a conclusion further supported by the presence of the drug-specific " $d$ " fragment at $m / z$ 393.195. The lack of prominent " $p$ " ions in the spectrum suggests linkage of aromatic carbon to the GSH sulfur. The MS/MS spectrum of THC4 exhibited a [M-H] ion at $m / z 678.334$, i.e., 38 Da higher than observed for THC2. This observation would be consistent with GSH adduction to a metabolite of 11-nor-9-carboxy- $\Delta^{9}$-THC containing a second carboxyl function. Furthermore, the appearance of " $"$ " ions at much higher intensity than " $k$ " ions, in addition to the absence of " $d$ " ions in this spectrum, suggests a linkage of the GSH sulfur to a benzylic carbon. Nevertheless, additional data are required to identify the exact nature of the metabolic modifications present in the THC3 and THC4 adducts and the location of the C-S linkage in each species.

\subsubsection{Task 2 Discussion}

This task explored the capability of selected drugs of abuse to form adducts with the tripeptide glutathione. The formed adducts result from covalent bonds between the nucleophilic sulfur in GSH and an electrophilic site on the parent drug or a metabolite. The formation of GSH-based adducts with drugs of abuse has only been sparingly reported in the literature, with available data limited to cocaine $^{61}$, MDMA and MDPV ${ }^{84}$, and morphine ${ }^{58}$. In these examples, MDMA was the only compound with reported GSH adducts formed by both the precursor 
drug and a metabolite. While previous work with these drugs has demonstrated the capability to form adducts with GSH, there is clearly a lack of available information on this phenomenon for other widely abused substances.

Of the $22 \mathrm{GSH}$ adducts observed in this study, nine have been previously reported in the literature (see Table 9 for references). The structures reported previously all have masses which match closely with those observed in spectra collected in the present study. ${ }^{58,61,80,81,84}$ A direct comparison of our spectral data with previous reports is possible for MDMA and MDPV, where negative mode MS ionization and analysis was also utilized. MS/MS data for fragments of GSH and GSH containing compounds and molecular ion exact masses observed for MDMA1, MDMA2, MDMA3, and MDPV1 in the present study agree with the data previously reported with negative mode analysis. ${ }^{84}$

Plausible structures are proposed for the majority of the previously unreported adducts identified in the present study, based upon HRMS accurate mass, MS/MS data, and likely metabolic transformations. However, for the OXY2, THC3, and THC4 adducts, the available data were insufficient to propose a structure with a high degree of confidence. The fragmentation patterns suggest that both OXY2 and THC4 have benzylic covalent thiol linkages, while THC3 likely contains an aromatic linkage. The molecular ion masses of THC3 and THC4 seem to suggest further metabolic modifications to the metabolite 11nor-9-carboxy- $\Delta^{9}$-THC prior to covalent adduction with GSH, such as a hydroxylation or further carboxylation. OXY2 also appears to have undergone 
additional metabolic modification prior to adduction, although the exact steps are unclear.

While NAL1 and OXY1 have an adduct mass which corresponds to the parent drug directly bound to GSH without any other modifications, the other structures proposed for GSH adducts in Table 9 represent adducts formed by one or more primary metabolites of the drug. One of the initial metabolic steps is likely to be hydroxylation, as is consistent with the proposed structures for CLZ1, DZP1, MDPV3, and NAL2. Hydroxylations are common oxidative steps in the metabolic pathways of CLZ, DZP, and MDPV, although this pathway has not been reported for NAL. ${ }^{129,133,134}$

Structures consistent with bond cleavage within the drug moiety were also observed for three of the adducts. CLZ3 is proposed to have lost the piperazine ring in addition to undergoing hydroxylation and rearomatization. While this particular metabolite has not previously been reported in the literature, there have been observations of modifications to the piperazine ring in CLZ, indicating that it may be a site of potential metabolic processes resulting in loss of piperazine ring. ${ }^{135}$ Loss of a piperazine ring has been exhibited in the metabolism of other heterocycle containing compounds such as aildenafil. ${ }^{136}$ Formation of MDPV2 is associated with loss of the methylene bridge and pyrrolidine moiety (via oxidative deamination) in addition to rearomatization, while MDPV3 may form via the same process in addition to a hydroxylation on the resultant alkyl chain. Demethylenation, common to either the orthocatechol or orthoquinone, and oxidative deamination of methylenedioxy type drugs has been 
reported in the literature. ${ }^{84,134}$ The orthoquinone formed by some methylenedioxy drugs has been reported to be the reactive metabolite responsible for toxicity. ${ }^{87}$ As mentioned previously, some of the drugs underwent a rearomatization step following GSH adduction and NIH shift (CLZ1, MDPV1, MDPV2, NAL1, OXY1, THC1, and THC2) while others did not (DZP1 and NAL2). This observation is similar to what has been generally reported in the literature involving NIH shifts ${ }^{19}$.

GSH adducts with $\Delta^{9}-\mathrm{THC}$ have not been previously reported. The proposed structures for THC1 and THC2 are consistent with adducts formed from the stable metabolites $11-\mathrm{OH}-\Delta^{9}$-THC and 11-nor-9-carboxy- $\Delta^{9}$-THC, respectively, with direct binding to $\mathrm{GSH}$. The fragmentation patterns and peak ratios discussed in the Results section indicate the thiol linkages appear to be benzylic for THC1 and aliphatic for THC2. The fact that these adducts are consistent with the stable metabolites implies that there may be a reactive intermediate formed, allowing for electrophilic interactions with the thiol moiety from GSH.

For the MS analysis of the GSH compounds in the present study, negative mode scanning was utilized. This was chosen primarily to facilitate analysis of adducted GSH fragmentation. GSH fragmentation patterns in negative mode are well-established in the literature, and in fact have been studied for a variety of covalent adducts, including those with aromatic, aliphatic, and benzylic linkages. ${ }^{88,110}$ The observed transitions also provided more prominent and clearer ion signals than those following initial analyses utilizing positive mode scanning. Additionally, many of the parent drugs included here do not produce prominent molecular ions or fragment ions in negative mode, thus minimizing 
potential interferences due to coelution with unreacted drug present in the assay mixture at significantly higher concentrations than the formed adducts. While positive mode analysis of the novel GSH adducts could provide additional data for structural confirmation, preliminary use of positive mode for several of the drugs in the present study did not provide useful fragmentation data. As other authors have reported, positive mode ionization is not always helpful for characterization of GSH adducts, particularly with certain drugs where doubly charged $[\mathrm{M}+2 \mathrm{H}]^{+}$ions are formed, as these do not allow for the neutral loss analysis which is generally utilized in positive mode GSH studies. ${ }^{110}$ Although negative ionization mode alone did not provide the data necessary for complete structural analysis of all adducts, this approach did provide sufficient information to determine a plausible adduct structure for most of the drugs under study. Unequivocal structural confirmation, including identification of regioselectivity of adduct formation where present, will require additional work with synthetic standards and further analysis by HRMS and definitive techniques such as NMR.

\subsection{Confirmation of adduction in human $\mathrm{Hb}$ by drugs of interest}

\subsubsection{Protein Adduction Assay Optimization}

Optimization of the in vitro assay system for protein adduction required numerous alterations from the previous assay used for GSH. Incubation time was increased to six hours to provide more time for interactions between the hemoglobin and the reactive metabolites. Several initial experiments utilized various products containing a dialysis membrane layer as an attempt to separate 
the hemoglobin from the metabolic system. The use of dialysis membrane microfuge inserts required significant scale up (approximately x10), and were deemed non cost-effective. Dialysis membrane layers were then abandoned in favor of ultra-fast centrifugation to pellet the HLM while the hemoglobin remained suspended in the solution. Concentrations of IAM and trypsin were established based on existing protocols in the literature regarding similar proteolytic digestions. ${ }^{137}$

\subsubsection{Analysis of Hemoglobin Trapping Assay Products}

The full scan analysis of the tryptic digestion assay supernatant produced a complex TIC (figure 28a). BioConfirm software was able to remove compounds not relevant to the formed tryptic peptides to produce a total compound chromatogram (TCC). The software then analyzed the TCC (Figure 28b) for expected peptides, peptide fragments, and any modifications allowed for in the sample processing method found in Figure 30 (full Bioconfirm method parameters found in Figure 29). Each drug-related adduct modification was manually input into the BioConfirm method based upon the observed masses from the GSH and synthetic peptide studies. Once completed, BioConfirm output a list of biomolecules found in the data. The MS data analyzed with BioConfirm produced positive results for the adduction of APAP, for the first time confirming via MS data what had been reported previously in the literature using radiolabeling and HPLC studies. ${ }^{125,138}$ In fact, APAP adduction was observed for both $\mathrm{Hb} \beta^{93}$ Cys and the less reactive $\mathrm{Hb} \beta^{112} \mathrm{Cys}$. Adduction was also observed via MS data for $\alpha-P V P, M E T H, N A L, O X Y$, and THC, producing a total of 11 
potential adducts. A summary of all of the BioConfirm data for all of these adducts can be found in Table 10. The mass difference, presented in ppm units, falls within an acceptable range for all but three of the 11 adducts observed in the MS studies. Generally, a TOF is capable of $<5$ ppm measurments ${ }^{139}$; only adducts 6,9 , and 11 fell outside of this range. Five of the potential adducts were also reported by BioConfirm to contain more than one modification on the analyzed peptide (i.e. an additional hydroxylation and/or acetylation). These additional modifications lower the certainty of the presence of adducts $5,6,9,10$, and 11, but was not enough to eliminate these as potential adducts.

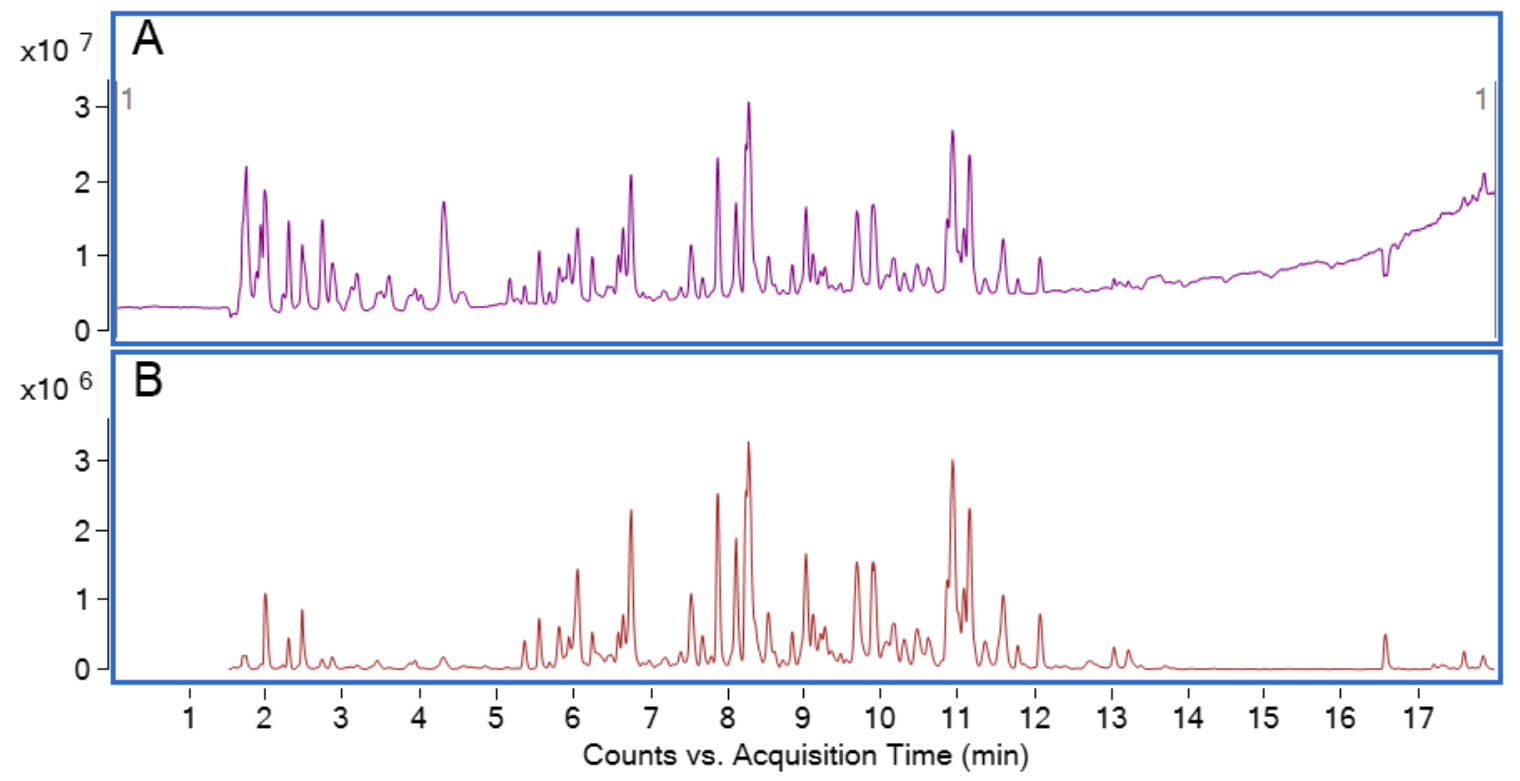

Figure 28. TIC (a) and TCC (b) for the APAP+Hb adduct. The removal of certain peaks was accomplished using the BioConfirm software. 


\section{Parameter}

\begin{tabular}{ll}
\hline Extraction algorithm & Small Molecules \\
Restrict $\mathrm{m} / \mathrm{z}$ to & $350-2000$ \\
Use peaks with height & 100 counts \\
Positive ions & $+\mathrm{H},+\mathrm{Na},+\mathrm{K},+\mathrm{NH}_{4}$ \\
Neutral losses & $\mathrm{H}_{2} \mathrm{O}$ \\
Isotope model & Peptides \\
Mass filters & none \\
Maximum number of peaks & 50 largest
\end{tabular}

Figure 29. BioConfirm method parameters.

\section{Value}

Small Molecules

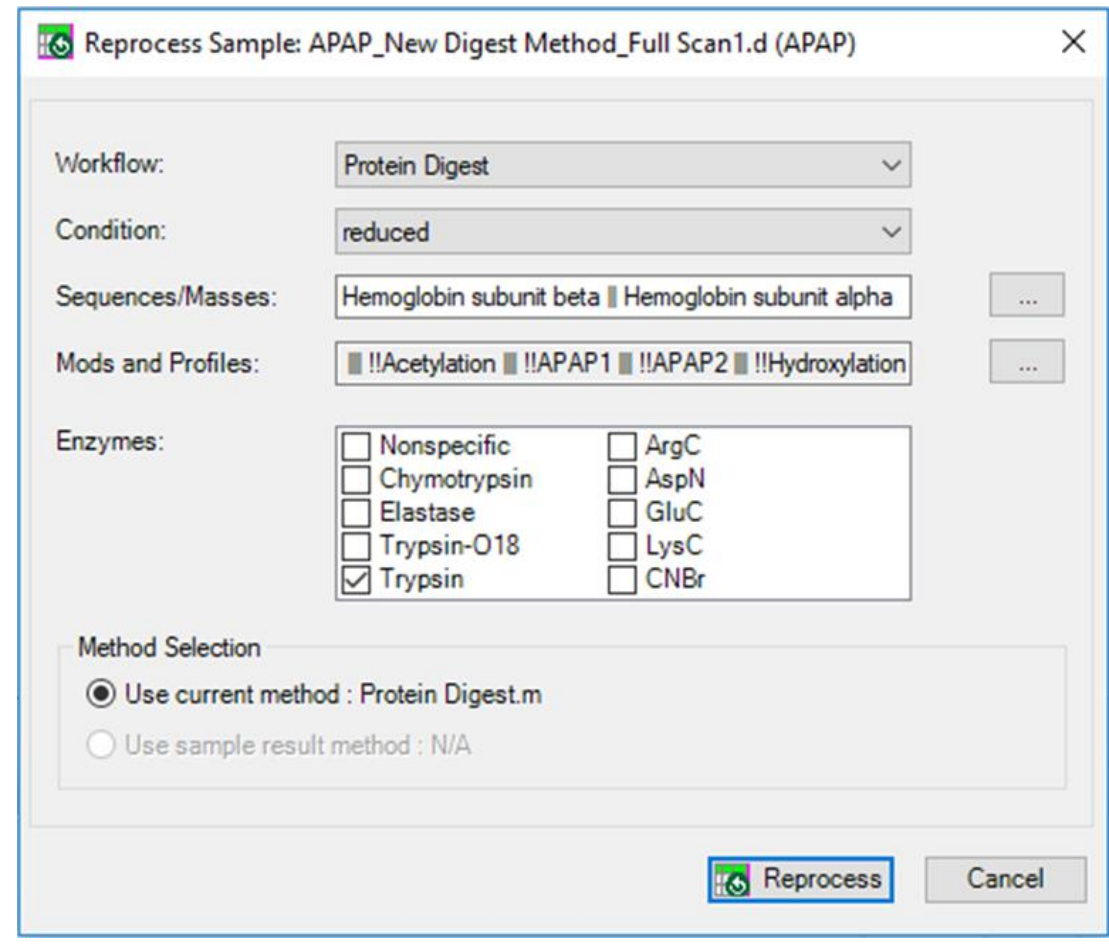

Figure 30. The BioConfirm sample processing method utilized for the adducted tryptic peptides. Modifications and profiles included the modifications for acetylation, hydroxylation, and the adducted drug moieties observed in the GSH experiments or synthetic peptide experiments performed by Dr. Carolina Moller (publication pending). 
Adduction

Adduct

Site
Other Mods
Adducted Peptide

Mass

\begin{tabular}{|c|c|c|c|c|c|}
\hline 1 & APAP1 & $\mathrm{Hb} \beta^{93}$ & none & 2.93 & 1569.718 \\
\hline 2 & APAP1 & $\mathrm{Hb} \beta^{112}$ & none & -3.29 & 1868.0069 \\
\hline 3 & $\alpha-P V P 1$ & $\mathrm{Hb} \beta^{93}$ & none & -3.63 & 1651.822 \\
\hline 4 & METH1 & $\mathrm{Hb} \beta^{93}$ & none & -4.63 & 1597.7632 \\
\hline 5 & METH1 & $\mathrm{Hb} \alpha^{104}$ & 1Hydrox.+1Acetyl. & 3.36 & 3171.7261 \\
\hline 6 & METH1 & $\mathrm{Hb} \beta^{93}$ & 1Acetyl. & 5.14 & 1609.7728 \\
\hline 7 & NAL1 & $\mathrm{Hb} \beta^{93}$ & none & -0.61 & 1759.8117 \\
\hline 8 & OXY1 & $\mathrm{Hb} \beta^{93}$ & none & 3.57 & 1733.8033 \\
\hline 9 & OXY1 & $\mathrm{Hb} \alpha^{104}$ & 1Hydrox.+1Acetyl. & 7.84 & 3337.7682 \\
\hline 10 & THC1 & $\mathrm{Hb} \beta^{93}$ & 1Hydrox.+1Acetyl. & 4.34 & $2914.4338^{*}$ \\
\hline 11 & THC2 & $\mathrm{Hb} \beta^{93}$ & 1Hydrox. & -7.16 & 1780.8466 \\
\hline
\end{tabular}

Table 10. Summary of MS data collected for $\mathrm{Hb}$ adducts. The asterisk for adduct 11 indicates a missed tryptic cleavage in the identified adduct.

\subsubsection{MS/MS Analysis of Hemoglobin Adducts}

Each drug which produced a potential adduct was additionally subjected to MS/MS analysis using the Auto MS/MS scan mode data. BioConfirm successfully identified MS/MS fragmentation for two of the adducts from Table 10, APAP1 modified $\mathrm{Hb} \beta^{93}$ and $\alpha-P V P 1$ modified $\mathrm{Hb} \beta^{93}$. While BioConfirm is capable of labeling individual amino acids and both $b$ and $y$ fragments in accordance with the a,b,c and $x, y, z$ nomenclature, it fails to label any other peaks which may be pertinent. Therefore, each spectrum was manually compared to a 
list of theoretical peaks provided by the MS-Product program of Protein Prospector (http://prospector.ucsf.edu). Protein Prospector is an online series of tools used for proteomic data mining specifically with mass spectrometry. The programs were developed by the University of California at San Francisco Mass Spectrometry Facility. The parameters used to compile the theoretical peak table can be found in Table 11 .

\begin{tabular}{|l|l|}
\hline Parameter & Value \\
\hline Sequence & $\begin{array}{l}\text { Based on hypothesized match done by BioConifrm } \\
\text { software } \\
\text { Modification represented by cysteine residue followed by } \\
\text { parentheses containing the exact mass of the modification }\end{array}$ \\
\hline N-term Sequence & a, b, c \\
\hline C-term Sequence & $\mathrm{x}, \mathrm{y}, \mathrm{z}$ \\
\hline Internal Fragment & $\mathrm{Allow}$ for internal fragmentation \\
\hline Neutral-loss & $-\mathrm{H}_{2} \mathrm{O}(\mathrm{S}, \mathrm{T}, \mathrm{E}, \mathrm{D})$ \\
Sequence & $-\mathrm{NH} 3(\mathrm{R}, \mathrm{K}, \mathrm{Q}, \mathrm{N})$ \\
\hline Peeling & $\mathrm{B}+\mathrm{H}_{2} \mathrm{O}(\mathrm{R}, \mathrm{H}, \mathrm{K})$ \\
\hline Sequence & \\
\hline Max Charge & 2 \\
\hline Mass Type & $\mathrm{Monoisotopic}$ \\
\hline Instrument & $\mathrm{ESI-Q}-\mathrm{TOF}$ \\
\hline
\end{tabular}

Table 11. Protein Prospector parameters 
In the BioConfirm data for the APAP adduct with $\mathrm{Hb}, 11$ peaks were identified in the MS/MS spectrum (Figure 31). Using the potential peak list populated by Protein Prospector, 18 additional peaks were identified. The resulting 29 peaks along with the corresponding fragment can be found in Table 12. The MS/MS data of the negative control unmodified peptide chain can be found in Figure 32.
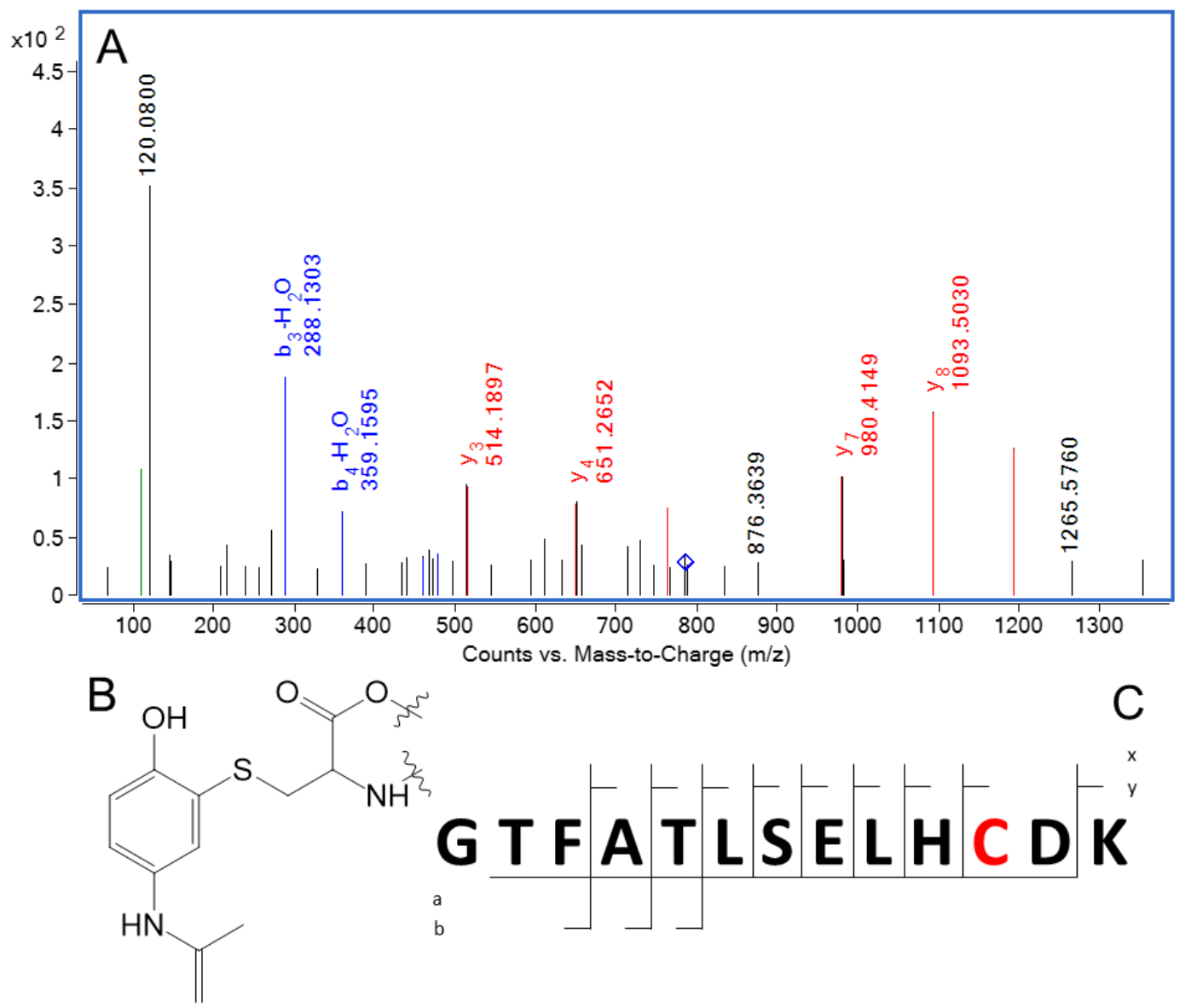

Figure 31. MS/MS identification of the adduct formed between APAPand $\mathrm{Hb} \beta^{93}$. (A) BioConfirm labeled MS/MS spectrum 11 peaks in the spectrum with the $b$ fragment in blue, the $y$ fragments in red, and the amino acid histidine shown in green. (B) Putative structure of APAP adduct. (C) Peptide fragmentation diagram for MS/MS data. 


\begin{tabular}{|c|c|c|c|}
\hline Fragment Mass & Fragment & Fragment Mass & Fragment \\
\hline 110.0713 & $\mathrm{H}$ & $593.3293^{*}$ & TFATLS-28 \\
\hline 120.0808 & $\mathrm{~F}$ & 633.2573 & $\mathrm{Y}_{4}-\mathrm{H}_{2} \mathrm{O}$ \\
\hline 145.0972 & AT-28 & 651.2678 & $\mathrm{y}_{4}$ \\
\hline 147.1128 & $\mathrm{y}_{1}$ & 729.2784 & $\mathrm{ELHC}(+149.06) \mathrm{D}-\mathrm{H}_{2} \mathrm{O}$ \\
\hline 217.0819 & SE & 747.3254 & $\mathrm{~V}_{5}-\mathrm{NH}_{3}$ \\
\hline $257.6081^{+2 *}$ & $\mathrm{y}_{3}^{+2}$ & 764.3519 & $\mathrm{y}_{5}$ \\
\hline 288.1343 & $\mathrm{~b}_{3}-\mathrm{H}_{2} \mathrm{O}$ & $785.8701^{+2}$ & $\mathrm{MH}^{+2}$ \\
\hline 359.1714 & $\mathrm{~b}_{4}-\mathrm{H}_{2} \mathrm{O}$ & 835.456 & TFATLSEL-28 \\
\hline 433.2445 & FATL & 876.368 & $\mathrm{Y}_{6}-\mathrm{NH}_{3}$ \\
\hline 439.23 & SELH-28 & 980.4265 & $y_{7}$ \\
\hline 460.2191 & $b_{5}-\mathrm{H}_{2} \mathrm{O}$ & 982.4993 & TFATLSELH- $\mathrm{H}_{2} \mathrm{O}$ \\
\hline 467.2249 & SELH & 1093.5106 & $\mathrm{y}_{8}$ \\
\hline 478.2296 & $b_{5}$ & 1194.5583 & $\mathrm{y}_{9}$ \\
\hline 496.1984 & $\mathrm{Y}_{3}-\mathrm{H}_{2} \mathrm{O}$ & 1265.5954 & $\mathrm{y}_{10}$ \\
\hline 514.2089 & $y_{3}$ & & \\
\hline
\end{tabular}

Table 12. Summary of MS/MS peak information for Hb+APAP adduct. Possible match peaks are indicated with an asterisk.

In the BioConfirm data for the a-PVP adduct with $\mathrm{Hb}, 3$ peaks were identified in the MS/MS spectrum (Figure 33). Using the potential peak list populated by Protein Prospector, 7 additional peaks were identified. The resulting 10 peaks along with the corresponding fragment can be found in Table 13. 


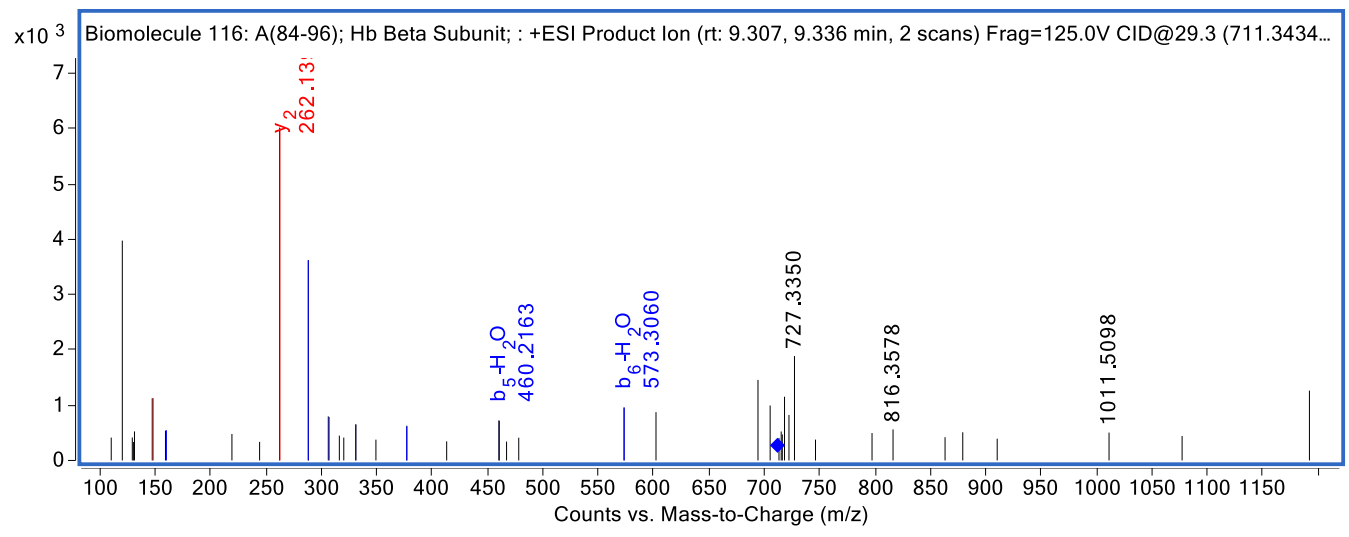

Figure 32. MS/MS data for the unmodified peptide containing the $\mathrm{Hb} \beta^{93}$ cysteine.

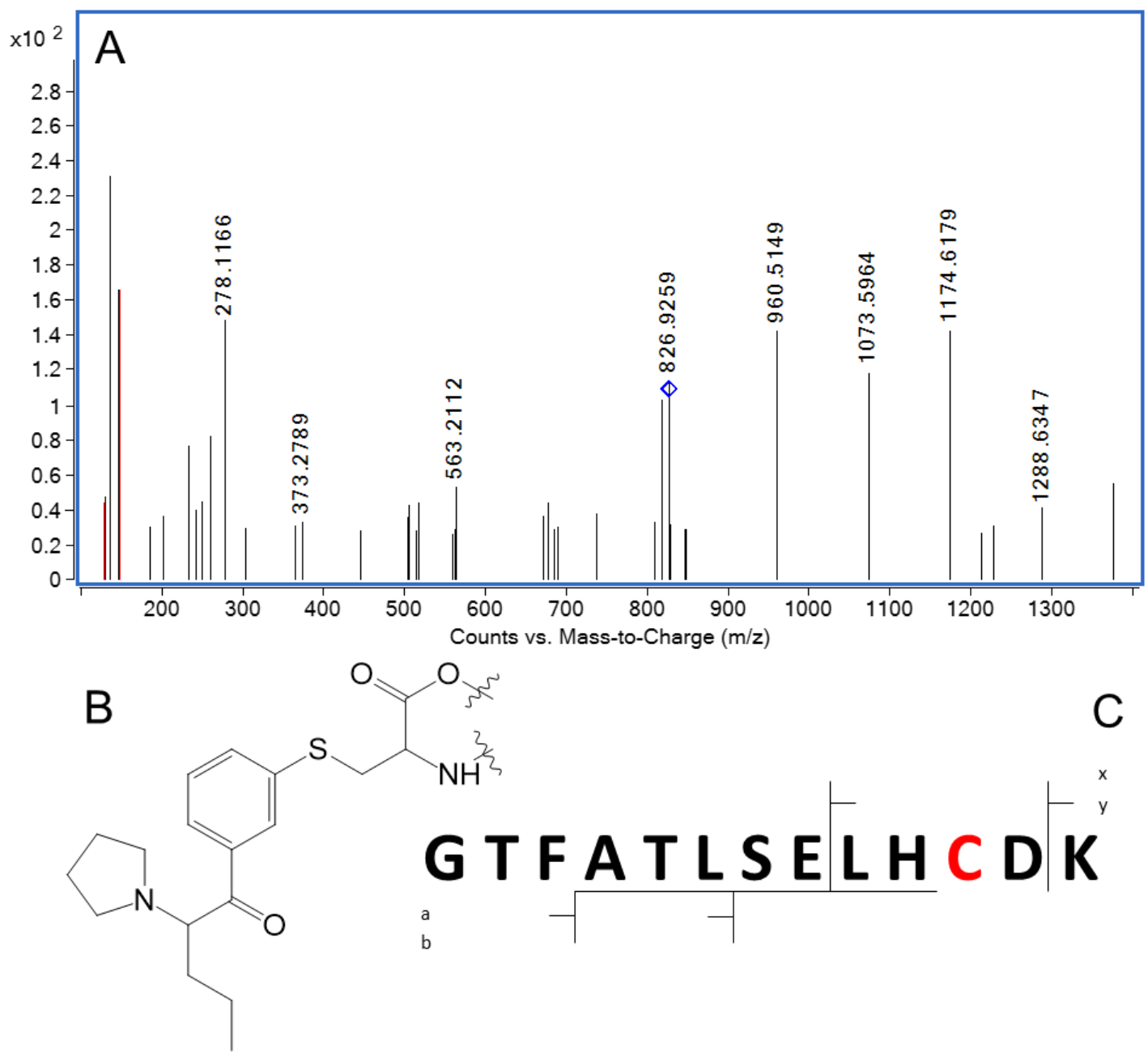

Figure 33. MS/MS identification of the adduct formed between $\alpha-P V P$ and $\mathrm{Hb} \beta^{93}$. (A) BioConfirm labeled MS/MS spectrum with 3 peaks identified in the spectrum, all $y$ fragments shown in red. (B) Putative structure of $\alpha$-PVP adduct. (C) Peptide fragmentation diagram for MS/MS data. 


\begin{tabular}{|c|c|}
\hline Fragment Mass & Fragment \\
\hline 129.1022 & K \\
\hline 130.0863 & $\mathrm{y}_{1}-\mathrm{NH}_{3}$ \\
\hline 147.1128 & $\mathrm{y}_{1}$ \\
\hline 278.1499 & $a_{3}$ \\
\hline 373.2082 & ATLS \\
\hline 562.2984 & LSELH- $\mathrm{H}_{2} \mathrm{O}$ \\
\hline $563.3188^{*}$ & $a_{6}$ \\
\hline $818.4068^{+2}$ & $\mathrm{MH}-\mathrm{NH}_{3}{ }^{+2}$ \\
\hline $826.9201^{+2}$ & $\mathrm{MH}^{+2}$ \\
\hline 846.4519 & $y_{5}$ \\
\hline
\end{tabular}

Table 13. Summary of MS/MS peak information for $\mathrm{Hb}+\alpha-P V P$ adduct. Possible match peaks are indicated with an asterisk.

\subsubsection{Task 3 Discussion}

Task 3 examined the capability of drugs of abuse to form covalent adducts with hemoglobin in vitro. As discussed in section 2.4.1, adducts formed between drugs of abuse and proteins is not a new concept, but the literature is limited. Few examples exist of studies analyzing these adducts, with the exceptions being ethanol, morphine, and cocaine..$^{52,53,59,65}$ Acetaminophen has also been shown via HPLC analysis to bind directly to hemoglobin. ${ }^{138}$ However, there has not been any mass spectrometric investigation into these formed adducts. Table 10 exhibits information supporting the formation of adducts formed between hemoglobin and six drugs of interest in this study; APAP, a-PVP, METH, NAL, 
OXY, and THC. The use of HRMS analysis increased the confidence in these data, despite the uncertainty regarding the adducts with $\Delta m / z \geq 5 \mathrm{ppm}$.

Additionally, the adducts listed as having more than one modification introduce another degree of uncertainty, but were still included as the BioConfirm software identified them. Each modification for a drug of abuse adduct had to be manually generated. An example of a manual modification setup for BioConfirm can be seen in Figure 34.

\begin{tabular}{|c|c|c|c|c|c|}
\hline \multicolumn{3}{|c|}{ Molecular formula } & \multicolumn{3}{|c|}{ Delta mass } \\
\hline Loss: & \multicolumn{2}{|l|}{$\mathrm{H}$} & \multicolumn{3}{|c|}{ Average: } \\
\hline Gain: & \multicolumn{2}{|c|}{$\mathrm{C} 8 \mathrm{H} 8 \mathrm{NO} 2$} & \multicolumn{2}{|c|}{ Mono: } & 149.04767 \\
\hline \multicolumn{6}{|c|}{ Amino acid specificity } \\
\hline$\square \mathrm{A}$ & $\square \mathrm{G}$ & $\square \mathrm{M}$ & $\square \mathrm{s}$ & \multicolumn{2}{|c|}{$\square$ N-Terminus } \\
\hline$\nabla c$ & $\square \mathrm{H}$ & $\square \mathrm{N}$ & $\square^{\top}$ & \multicolumn{2}{|c|}{$\square$ c-Terminus } \\
\hline$\square \mathrm{D}$ & $\square$ & $\square \mathrm{P}$ & $\square v$ & & \\
\hline$\square \mathrm{E}$ & $\square \mathrm{k}$ & $\square Q$ & $\square \mathrm{w}$ & & \\
\hline$\square \mathrm{F}$ & $\square \mathrm{L}$ & $\square R$ & $\square Y$ & & \\
\hline \multicolumn{6}{|c|}{$\mathrm{N}$-linked glycosylation recognizes NXS $T$ consensus sites } \\
\hline \multicolumn{6}{|c|}{ O-linked glycosylation recognizes S/T } \\
\hline \multicolumn{6}{|c|}{$\square$ Apply modification limits } \\
\hline \multicolumn{4}{|c|}{ Maximum modifications per biomolecule: } & \\
\hline
\end{tabular}

Figure 34. Example of custom modification settings used for BioConfirm analysis

An important note regarding all of the observed adducts is that they are drug specific. Rather than a portion of a drug being covalently bound, such as a phenyl ring or amine, the entire drug moiety is bound to the cysteine of $\mathrm{Hb}$. This implies that any long-term biomarkers arising from the use of protein adducts will 
be indicative of a specific drug, rather than a class of drugs. It also implies that the use of protein adducts as biomarkers could be used in confirmatory tests, rather than screening. There may also be more adducts formed than what was detected by the QTOF instrument, including those formed with different drugs of interest. The formed adducts are often produced at very low concentrations, and it is possible that the instrument, based upon user parameters, disregarded adduct peaks in favor of fragments with significantly higher concentration.

The MS/MS data provide further evidence of the covalent adduction by APAP and $\alpha-P V P$. Both of these drugs were covalently bound to $\mathrm{Hb} \beta^{93}$, rather than the other two possible cysteines. This is unsurprising since $\mathrm{Hb} \beta^{93}$ is the most reactive cysteine residue found in $\mathrm{Hb}^{99}$, as it lies largely exposed on the surface of the protein (Figure 35).

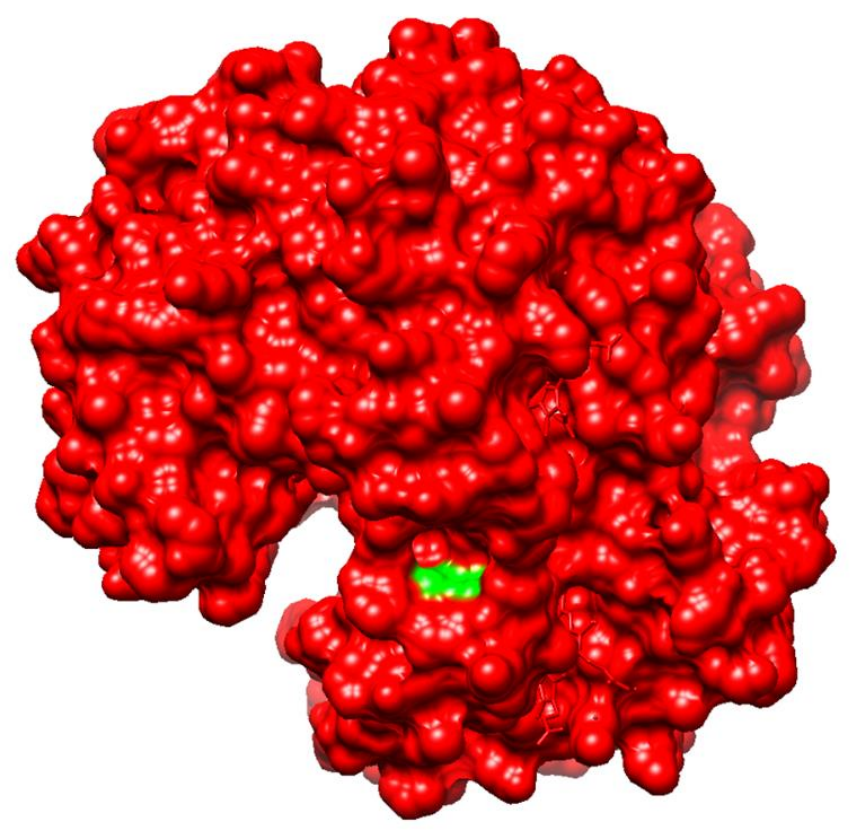


Figure 35. Space-filled model of $\mathrm{Hb}$ with $\mathrm{Hb} \beta^{93}$ highlighted in green.

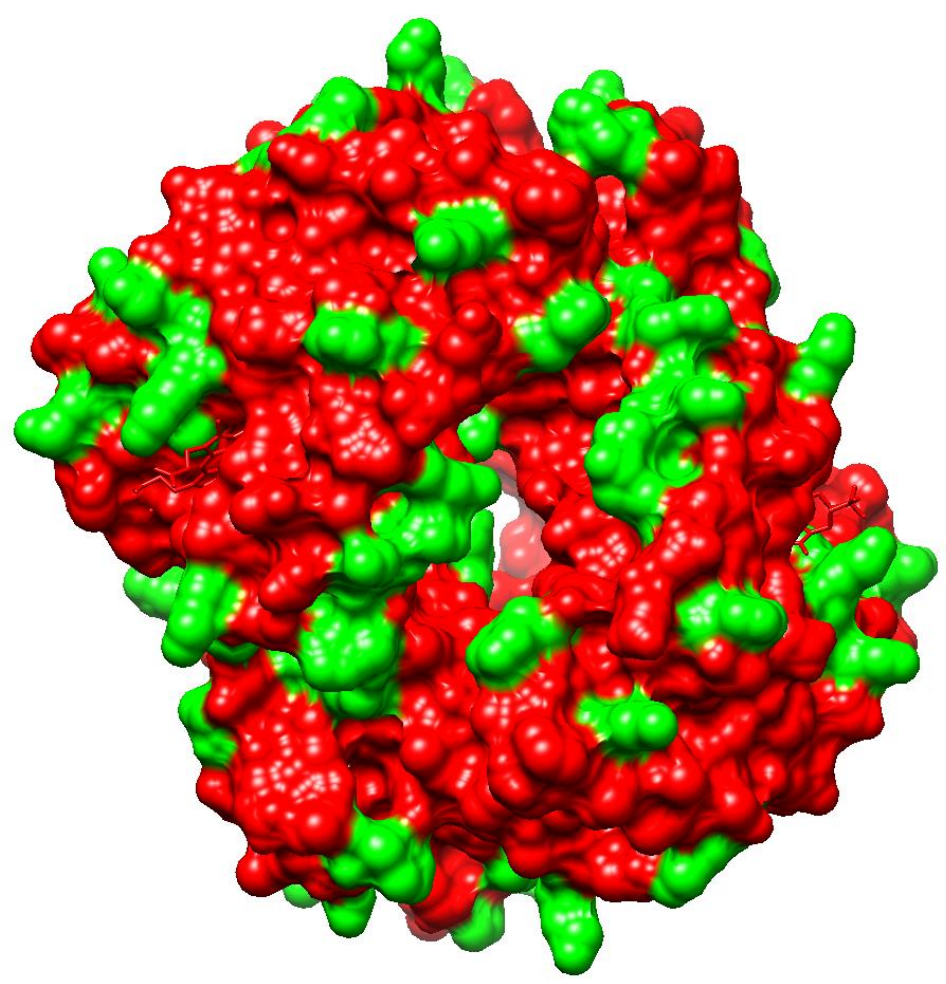

Figure 36. Space-filled model of $\mathrm{Hb}$ with His and Lys residues highlighted in green.

MS/MS data were unable to be collected for nine of the eleven adducts observed in the MS analysis. This could be the result of low concentrations of the formed adducts. As mentioned earlier in this section, the adducts are present in much lower concentrations than the other components of the assay analyzed by the instrument. Parameters of the QTOF analytical method may have accidentally excluded some of the adducts. To be certain, exclusion lists would need to be created containing the masses of the non-adducted peptides and any other irrelevant molecules.

While $\mathrm{Hb} \beta^{93}$ is the most reactive cysteine, it is possible that other adducts are formed with additional nucleophilic amino acid residues on the hemoglobin 
molecule. As visible in Figure 36, there are many other nucleophilic sites in the hemoglobin protein. These include the amino acids histidine and lysine, both of which have been shown to form covalent adducts with electrophiles. ${ }^{73}$ Any of these adducts would have been missed since cysteine was specifically targeted in the BioConfirm parameters allowed for modifications (see Figure 34). Further work would therefore be required to determine any additional sites of possible adduction by the drugs of interest. 


\section{Summary and Prospect}

The goal of this research was to investigate the potential of adduct formation between selected drugs of abuse and the blood protein hemoglobin. This was accomplished through a series of steps, including the determination of fragmentation of the parent drugs and the analysis of adduction with the small tripeptide glutathione. This work successfully compiled a list of common fragments for all 16 of the drugs of interest, which, although not thoroughly utilized for this work, may provide insight in the future to potential fragmentation by adducted drug moieties. This work also identified and characterized 22 adducts with GSH, 12 of which were novel. Although structural determination was not a focus of this research, plausible structures for 9 of the 12 novel adducts were proposed. Positive ionization analysis of the GSH adducts may provide more details into the missing structures and aid in confirming the proposed structures of the putative adducts. Lastly, this research was able to successfully collect HRMS data for eleven $\mathrm{Hb}$ adducts with the drugs of interest and MS/MS data for two adducts, thus confirming the adduction by $\alpha$-PVP and APAP.

This research has laid the groundwork for future expansion into the topic of covalent protein adduction by drugs of abuse. One major area of potential future work is the investigation of other proteins, specifically human serum albumin (HSA). HSA, like $\mathrm{Hb}$, is a common blood protein with a free cysteine residue at $\mathrm{Cys}^{34}$. HSA is also produced in the liver, where much of the metabolism in the body takes place. This could potentially drive the adduction of 
short-lived reactive metabolites with HSA. There is also a plethora of literature regarding the adduction of compounds to HSA. The metabolically generated carcinogen ( \pm )-r-7,t-8-dihydroxy-c-9,c-10-epoxy-7,8,9,10-tetrahydrobenze (antiBaPDE) has been shown via fluorescence line narrowing spectroscopy to produce multiple adducts with HSA. ${ }^{140}$ Adducts have also been observed between HSA and atrazine, a common herbicide. ${ }^{141}$ Finally, the structurally similar bovine serum albumin (BSA) has been shown to form adducts with APAP. ${ }^{123} \mathrm{HSA}$, much like $\mathrm{Hb}$, is not limited to cysteine as possible sites of adduction, as studies have shown the nucleophilic properties of multiple histidine and lysine residues promote adduction. ${ }^{73}$

The concentration of many protein adducts has been shown to be very low, with in vitro studies reporting that a majority of analyzed compounds exhibited protein binding with less than $10 \%$ of the overall metabolome. ${ }^{142}$ APAP, a drug observed in the present study to have the highest adduct concentrations, was observed modifying proteins with $3.1 \%$ of the overall metabolome. ${ }^{142}$ In vivo studies have shown even lower concentrations present, with Rubino et al. reporting that hemoglobin modified by a mass 200 Daltons or higher was present at concentrations of $1 \mathrm{ppb}$ or lower. ${ }^{143} \mathrm{~A}$ solution to this limitation would be to carry out an enrichment procedure by isolating the modified protein before analysis.

This work has many implications in the field of forensics. At the present time, hair analysis is the only available method for long-term detection of illicit drug use. However, hair analysis suffers from methodological and interpretive 
challenges, and the mechanisms by which most drugs incorporate into hair are not clearly established. ${ }^{21}$ Additionally, current blood and urine biomarkers are removed from the body after just a few days, restricting the window of detection for many drugs of abuse. The use of protein adducts as long-term biomarkers of exposure may be used to supplement existing analytical methods to ensure complete detection of drugs of abuse. As all of the thusly identified proteins adducts are specific to individual drugs, it may be possible to confirm not only the class of drugs consumed but also the exact drug consumed based upon the MS/MS data collected for an individual's sample. This specificity combined with the overall stability makes long-term biomarkers of exposure a favorable and effective application of protein adducts formed by drugs of abuse. 


\section{Reference List}

1. Center for Behavioral Health Statistics and Quality. 2014 national survey on drug use and health: detailed tables; Substance Abuse and Mental Health Services Administration: Rockville, MD, 15.

2. Karch, S. B.; Drummer, O. H. Karch's Pathology of Drug Abuse; CRC Press: Boca Raton, 2016; Vol. 5.

3. Goldstein, R. A.; DesLauriers, C.; Burda, A. M. Cocaine: history, social implications, and toxicity-a review. Disease-a-Month 2009, 55 (1), 6-38.

4. Orsolini, L.; Papanti, D.; Vecchiotti, R.; Valchera, A.; Corkery, J.; Schifano, F. Novel psychoactive substances. European Psychiatry 33, S59S60.

5. Drug Enforcement Administration . Drug schedules. 2018. 3-20-2018. https://www.dea.gov/druginfo/ds.shtml

6. Abadinsky, H. Drug use and abuse: a comprehensive introduction; Thomson Higher Education: Belmont, 2008.

7. Coomber, R.; McElrath, K.; Measham, F.; Moore, K. Key Concepts in Drugs and Society; SAGE Publications Ltd: London, 2013.

8. Dasgupta, A. A Health Educator's Guide to Understanding Drugs of Abuse Testing; Jones and Bartlett Publishers, LLC: Sudbury, 2010.

9. Isralowitz, R. E.; Myers, P. L. Illicit Drugs; Greenwood: Santa Barbara, 2011.

10. Balhara, Y. P. S. Drug classifications. 2018. 3-20-2018. https://luxury.rehabs.com/drug-abuse/classifications/

11. Kumar, A.; Singla, C.; Singla, D. Drug metabolism. International Journal of Advances in Engineering Sciences 1[1], 67-68. 2011.

12. Testa, B.; Krämer, S. D. The biochemistry of drug metabolism - An introduction. Chemistry \& Biodiversity 2007, 4 (3), 257-405.

13. Newcomb, M.; Hollenberg, P. F.; Coon, M. J. Multiple mechanisms and multiple oxidants in P450-catalyzed hydroxylations. Archives of Biochemistry and Biophysics 2003, 409 (1), 72-79. 
14. Davydov, D. R. Microsomal monooxygenase in apoptosis: another target for cytochrome c signaling? Trends in Biochemical Sciences 2001, 26 (3), 155-160.

15. Auclair, K.; Hu, Z.; Little, D. M.; Ortiz de Montellano, P. R.; Groves, J. T. Revisiting the mechanism of P450 enzymes with the radical clocks norcarane and spiro[2,5]octane. Journal of the American Chemical Society 2002, 124 (21), 6020-6027.

16. Zangar, R. C.; Davydov, D. R.; Verma, S. Mechanisms that regulate production of reactive oxygen species by cytochrome P450. Toxicology and Applied Pharmacology 2004, 199 (3), 316-331.

17. Jerina, D. M.; Daly, J. W. Arene oxides: A new aspect of drug metabolism. Science 1974, 185 (4151), 573.

18. Ortiz de Montellano, P. R.; Nelson, S. D. Rearrangement reactions catalyzed by cytochrome P450s. Archives of Biochemistry and Biophysics 2011, 507 (1), 95-110.

19. Peter Guengerich, F. Cytochrome P450 oxidations in the generation of reactive electrophiles: epoxidation and related reactions. Archives of Biochemistry and Biophysics 2003, 409 (1), 59-71.

20. Fraser, A. D.; Zamecnik, J.; Keravel, J.; McGrath, L.; Wells, J. Experience with urine drug testing by the Correctional Service of Canada. Forensic Science International 2001, 121 (1), 16-22.

21. Wennig, R. Potential problems with the interpretation of hair analysis results. Forensic Science International 2000, 107 (1-3), 5-12.

22. Kraemer, T.; Maurer, H. H. Determination of amphetamine, methamphetamine and amphetamine-derived designer drugs or medicaments in blood and urine. Journal of Chromatography B: Biomedical Sciences and Applications 1998, 713 (1), 163-187.

23. Ishida, T.; Kudo, K.; Inoue, H.; Tsuji, A.; Kojima, T.; Ikeda, N. Rapid screening for and simultaneous semiquantitative analysis of thirty abused drugs in human urine samples using gas chromatographymass spectrometry. Journal of Analytical Toxicology 2006, 30 (7), 468-477.

24. Schwartz, J. G.; Garriott, J. C.; Somerset, J. S.; Igler, E. J.; Rodriguez, R.; Orr, M. D. Measurements of fentanyl and sufentanil in blood and urine after surgical application. Implication in detection of abuse. The American Journal of Forensic Medicine and Pathology 1994, 15 (3), 236-241. 
25. Moffat, A. C. Combined HPLC-RIA and HPLC-EMIT for the analysis of drugs of abuse in blood and urine. Swedish Pharmaceutical: Stockholm, 1978; p 482.

26. Trefz, P.; Kamysek, S.; Fuchs, P.; Sukul, P.; Schubert, J. K.; Miekisch, W. Drug detection in breath: non-invasive assessment of illicit or pharmaceutical drugs. Journal of Breath Research 2017, 11 (2), 024001.

27. Barbosa, J.; Faria, J.; Carvalho, F.; Pedro, M.; Queiros, O.; Moreira, R.; Dinis-Oliveira, $R$. Hair as an alternative matrix in bioanalysis. Bioanalysis 2013, 5 (8), 895-914.

28. Verstraete, A. G. Detection times of drugs of abuse in blood, urine, and oral fluid. Therapeutic Drug Monitoring 2004, 26 (2), 200-205.

29. American Addiction Centers . Detection window for drugs of sause. 2018. https://americanaddictioncenters.org/blog/detection-window/

30. Vogliardi, S.; Tucci, M.; Stocchero, G.; Ferrara, S. D.; Favretto, D. Sample preparation methods for determination of drugs of abuse in hair samples: A review. Analytica Chimica Acta 2015, 857, 1-27.

31. Henderson, G. L. Mechanisms of drug incorporation into hair. Forensic Science International 1993, 63 (1), 19-29.

32. Nakahara, Y. Hair analysis for abused and therapeutic drugs. Journal of Chromatography B: Biomedical Sciences and Applications 1999, 733 (1), 161-180.

33. Koren, G.; Klein, J.; Forman, R.; Graham, K. Hair analysis of cocaine: differentiation between systemic exposure and external contamination. The Journal of Clinical Pharmacology 1992, 32 (7), 671-675.

34. Blank, D. L.; Kidwell, D. A. External contamination of hair by cocaine: an issue in forensic interpretation. Forensic Science International 1993, 63 (1), 145-156.

35. Spiehler, V.; Levine, B. Pharmacokinetics and Pharmacodynamics. In Principles of Forensic Toxicology, 2nd ed.; Levine, B., Ed.; AACC Press: Washington, DC, 2006; pp 47-63.

36. Grune, T.; Reinheckel, T.; Davies, K. J. Degradation of oxidized proteins in mammalian cells. The FASEB Journal 1997, 11 (7), 526-534. 
37. Grune, T.; Shringarpure, R.; Sitte, N.; Davies, K. Age-related changes in protein oxidation and proteolysis in mammalian cells. The Journals of Gerontology Series A: Biological Sciences and Medical Sciences 2001, 56 (11), B459-B467.

38. Viner, R. I.; Williams, T. D.; Schneich, C. Peroxynitrite modification of protein thiols: oxidation, nitrosylation, and S-glutathiolation of functionally important cysteine residue(s) in the sarcoplasmic Reticulum Ca-ATPase. Biochemistry 1999, 38 (38), 12408-12415.

39. Tien, M.; Berlett, B. S.; Levine, R. L.; Chock, P. B.; Stadtman, E. R. Peroxynitrite-mediated modification of proteins at physiological carbon dioxide concentration: $\mathrm{pH}$ dependence of carbonyl formation, tyrosine nitration, and methionine oxidation. Proceedings of the National Academy of Sciences 1999, 96 (14), 7809-7814.

40. Shacter, E. Quantification and significance of protein oxidation in biological samples. Drug Metabolism Reviews 2000, 32 (3-4), 307326.

41. Nadkarni, D. V.; Sayre, L. M. Structural definition of early lysine and histidine adduction chemistry of 4-hydroxynonenal. Chemical Research in Toxicology 1995, 8 (2), 284-291.

42. Chondrogianni, N.; Petropoulos, I.; Grimm, S.; Georgila, K.; Catalgol, B.; Friguet, B.; Grune, T.; Gonos, E. S. Protein damage, repair and proteolysis. Molecular Aspects of Medicine 2014, 35, 1-71.

43. Fieser, L. F. Carcinogenic activity, structure, and chemical reactivity of polynuclear aromatic hydrocarbons. The American Journal of Cancer 1938, 34 (1), 37.

44. Miller, E. C.; Miller, J. A. The presence and significance of bound aminoazo dyes in the livers of rats fed $p$ dimethylaminoazobenzene. Cancer Research 1947, 7 (7), 468.

45. Evans, D. C.; Watt, A. P.; Nicoll-Griffith, D. A.; Baillie, T. A. Drug-protein adducts: an industry perspective on minimizing the potential for drug bioactivation in drug discovery and development. Chemical Research in Toxicology 2004, 17 (1), 3-16.

46. Brodie, B. B.; Reid, W. D.; Cho, A. K.; Sipes, G.; Krishna, G.; Gillette, J. R. Possible mechanism of liver necrosis caused by aromatic organic compounds. Proceedings of the National Academy of Sciences 1971, 68 (1), 160-164. 
47. Gillette, J. R.; Mitchell, J. R.; Brodie, B. B. Biochemical mechanisms of drug toxicity. Annual Review of Pharmacology 1974, 14 (1), 271288.

48. Skipper, P. L.; Bryant, M. S.; Tannenbaum, S. R.; Groopman, J. D. Analytical methods for assessing exposure to 4-aminobiphenyl based on protein adduct formation. Journal of Occupational Medicine. : Official Publication of the Industrial Medical Association 1986, 28 (8), 643-646.

49. Babu, S. R.; Lakshmi, V. M.; Huang, G. P.-W.; Zenser, T. V.; Davis, B. B. Glucuronide conjugates of 4-aminobiphenyl and its $\mathrm{N}$-hydroxy metabolites: $\mathrm{pH}$ stability and synthesis by human and dog liver. Biochemical Pharmacology 1996, 51 (12), 1679-1685.

50. Tornqvist, M.; Kautiainen, A.; Gatz, R. N.; Ehrenberg, L. Hemoglobin adducts in animals exposed to gasoline and diesel exhausts 1. Alkenes. Journal of Applied Toxicology 1988, 8 (3), 159-170.

51. Christakopoulos, A.; Bergmark, E.; Zorcec, V.; Norppa, H.; M+ñkiPaakkanen, J.; Osterman-Golkar, S. Monitoring occupational exposure to styrene from hemoglobin adducts and metabolites in blood. Scandinavian Journal of Work, Environment \& Health 1993, 255-263.

52. Donohue, T. M.; Tuma, D. J.; Sorrell, M. F. Acetaldehyde adducts with proteins: Binding of [14C]acetaldehyde to serum albumin. Archives of Biochemistry and Biophysics 1983, 220 (1), 239-246.

53. San George, R. C.; Hoberman, H. D. Reaction of acetaldehyde with hemoglobin. Journal of Biological Chemistry 1986, 261 (15), 68116821.

54. Nicholls, R.; de Jersey, J.; Worrall, S.; Wilce, P. Modification of proteins and other biological molecules by acetaldehyde: Adduct structure and functional significance. International Journal of Biochemistry 1992, 24 (12), 1899-1906.

55. Braun, K. P.; Pavlovich, J. G.; Jones, D. R.; Peterson, C. M. Stable acetaldehyde adducts: structural characterization of acetaldehyde adducts of human hemoglobin $\mathrm{N}$-terminal B-globin chain peptides. Alcoholism: Clinical and Experimental Research 1997, 21 (1), 4043.

56. Schneider, K. Covalent protein adduction by drugs of abuse. Florida International University, Feb 2013. 
57. Yamano, S.; Takahashi, A.; Todaka, T.; Toki, S. In vivo and in vitro formation of morphinone from morphine in rat. Xenobiotica 1997, 27 (7), 645-656.

58. Todaka, T.; Ishida, T.; Kita, H.; Narimatsu, S.; Yamano, S. Bioactivation of morphine in human liver: isolation and identification of morphinone, a toxic metabolite. Biological and Pharmaceutical Bulletin 2005, 28 (7), 1275-1280.

59. Nagamatsu, K.; Kido, Y.; Terao, T.; Ishida, T.; Toki, S. Studies on the mechanism of covalent binding of morphine metabolites to proteins in mouse. Drug Metabolism and Disposition 1983, 11 (3), 190-194.

60. Correia, M. A.; Krowech, G.; Caldera-Munoz, P.; Yee, S. L.; Straub, K.; Castagnoli Jr, N. Morphine metabolism revisited. II. Isolation and chemical characterization of a glutathionylmorphine adduct from rat liver microsomal preparations. Chemico-Biological Interactions 1984, 51 (1), 13-24.

61. Schneider, K. J.; DeCaprio, A. P. Covalent thiol adducts arising from reactive intermediates of cocaine biotransformation. Chemical Research in Toxicology 2013, 26 (11), 1755-1764.

62. Kovacic, P. Role of oxidative metabolites of cocaine in toxicity and addiction: oxidative stress and electron transfer. Medical Hypotheses 2005, 64 (2), 350-356.

63. Ndikum-Moffor, F. M.; Roberts, S. M. Cocaine-protein targets in mouse liver. Biochemical Pharmacology 2003, 66 (1), 105-113.

64. Ndikum-Moffor, F. M.; Munson, J. W.; Bokinkere, N. K.; Brown, J. L.; Richards, N.; Roberts, S. M. Immunochemical detection of hepatic cocaine-protein adducts in mice. Chemical Research in Toxicology 1998, 11 (3), 185-192.

65. Deng, S. X.; Bharat, N.; Fischman, M. C.; Landry, D. W. Covalent modification of proteins by cocaine. Proceedings of the National Academy of Sciences 2002, 99 (6), 3412-3416.

66. Kloss, M. W.; Rosen, G. M.; Rauckman, E. J. Cocaine-mediated hepatotoxicity a critical review. Biochemical Pharmacology 1984, $33(2), 169-173$.

67. Bornheim, L. M. Effect of cytochrome P450 inducers on cocaine-mediated hepatotoxicity. Toxicology and Applied Pharmacology 1998, 150 (1), 158-165. 
68. LoPachin, R. M.; Gavin, T.; DeCaprio, A.; Barber, D. S. Application of the hard and soft, acids and bases (HSAB) theory to toxicant-target interactions. Chemical Research in Toxicology 2012, 25 (2), 239251.

69. LoPachin, R. M.; Gavin, T. Reactions of electrophiles with nucleophilic thiolate sites: relevance to pathophysiological mechanisms and remediation. Free Radical Research 2016, 50 (2), 195-205.

70. Jaramillo, P.; Perez, P.; Contreras, R.; Tiznado, W.; Fuentealba, P. Definition of a nucleophilicity scale. The Journal of Physical Chemistry A 2006, 110 (26), 8181-8187.

71. Coles, B. Effects of modifying structure on electrophilic reactions with biological nucleophiles. Drug Metabolism Reviews 1984, 15 (7), 1307-1334.

72. Schultz, T. W.; Carlson, R. E.; Cronin, M. T. D.; Hermens, J. L. M.; Johnson, R.; O'Brien, P. J.; Roberts, D. W.; Siraki, A.; Wallace, K. B.; Veith, G. D. A conceptual framework for predicting the toxicity of reactive chemicals: modeling soft electrophilicity. SAR and QSAR in Environmental Research 2006, 17 (4), 413-428.

73. Thompson, V. R.; DeCaprio, A. P. Covalent adduction of nitrogen mustards to model protein nucleophiles. Chemical Research in Toxicology 2013, 26 (8), 1263-1271.

74. LoPachin, R. M.; Barber, D. S. Synaptic cysteine sulfhydryl groups as targets of electrophilic neurotoxicants. Toxicological Sciences 2006, 94 (2), 240-255.

75. Mah, R.; Thomas, J. R.; Shafer, C. M. Drug discovery considerations in the development of covalent inhibitors. Bioorganic \& Medicinal Chemistry Letters 2014, 24 (1), 33-39.

76. Guterman, L. Covalent drugs form long-lived ties. Chemical \& Engineering News 2011, 89 (36), 19.

77. Robertson, J. G. Mechanistic basis of enzyme-targeted drugs. Biochemistry 2005, 44 (15), 5561-5571.

78. Bauer, R. A. Covalent inhibitors in drug discovery: from accidental discoveries to avoided liabilities and designed therapies. Drug Discovery Today 2015, 20 (9), 1061-1073. 
79. Shimizu, E.; Hashimoto, K.; Komatsu, N.; lyo, M. Roles of endogenous glutathione levels on 6-hydroxydopamine-induced apoptotic neuronal cell death in human neuroblastoma SK-N-SH cells. Neuropharmacology 2002, 43 (3), 434-443.

80. Dahlin, D. C.; Miwa, G. T.; Lu, A. Y.; Nelson, S. D. N-acetyl-pbenzoquinone imine: a cytochrome P-450-mediated oxidation product of acetaminophen. Proceedings of the National Academy of Sciences of the United States of America 1984, 81 (5), 1327-1331.

81. Zhu, M.; Ma, L.; Zhang, H.; Humphreys, W. G. Detection and structural characterization of glutathione-trapped reactive metabolites using liquid chromatography - high-resolution mass spectrometry and mass defect filtering. Analytical Chemistry 2007, 79 (21), 83338341.

82. DeCaprio, A. P. The toxicology of hydroquinone - relevance to occupational and environmental exposure. Critical Reviews in Toxicology 1999, 29 (3), 283-330.

83. Blair, I. A. Analysis of endogenous glutathione-adducts and their metabolites. Biomedical Chromatography : BMC 2010, 24 (1), 2938.

84. Meyer, M. R.; Richter, L. H. J.; Maurer, H. H. Methylenedioxy designer drugs: Mass spectrometric characterization of their glutathione conjugates by means of liquid chromatography-high-resolution mass spectrometry/mass spectrometry and studies on their glutathionyl transferase inhibition potency. Analytica Chimica Acta 2014, 822, 37-50.

85. Gomez-Lechon, M. J.; Tolosa, L.; Donato, M. T. Metabolic activation and drug-induced liver injury: in vitro approaches for the safety risk assessment of new drugs. Journal of Applied Toxicology 2016, 36 (6), 752-768.

86. Inoue, K.; Shibata, Y.; Takahashi, H.; Ohe, T.; Chiba, M.; Ishii, Y. A trapping method for semi-quantitative assessment of reactive metabolite formation using [35S]cysteine and [14C]cyanide. Drug Metabolism and Pharmacokinetics 2009, 24 (3), 245-254.

87. Kalgutkar, A. S. Handling reactive metabolite positives in drug discovery: What has retrospective structure - toxicity analyses taught us? Chemico-Biological Interactions 2011, 192 (1-2), 46-55. 
88. Xie, C.; Zhong, D.; Chen, X. A fragmentation-based method for the differentiation of glutathione conjugates by high-resolution mass spectrometry with electrospray ionization. Analytica Chimica Acta 2013, 788, 89-98.

89. Yamaoka, T.; Kitamura, Y. Characterization of a highly sensitive and selective novel trapping reagent, stable isotope labeled glutathione ethyl ester, for the detection of reactive metabolites. Journal of Pharmacological and Toxicological Methods 2015, 76, 83-95.

90. Castro-Perez, J.; Plumb, R.; Liang, L.; Yang, E. A high-throughput liquid chromatography/tandem mass spectrometry method for screening glutathione conjugates using exact mass neutral loss acquisition. Rapid Communications in Mass Spectrometry: An International Journal Devoted to the Rapid Dissemination of Up-to-the-Minute Research in Mass Spectrometry 2005, 19 (6), 798-804.

91. Hiramatsu, M. A. S. A.; Kumagai, Y. O. S. H.; Unger, S. E.; Cho, A. K. Metabolism of methylenedioxymethamphetamine: formation of dihydroxymethamphetamine and a quinone identified as its glutathione adduct. Journal of Pharmacology and Experimental Therapeutics 1990, 254 (2), 521-527.

92. Jian, W.; Liu, H. F.; Zhao, W.; Jones, E.; Zhu, M. Simultaneous screening of glutathione and cyanide adducts using precursor ion and neutral loss scans-dependent product ion spectral acquisition and data mining tools. Journal of the American Society for Mass Spectrometry 2012, 23 (5), 964-976.

93. Parker, C. E.; De Wit, J. S. M.; Smith, R. W.; Gopinathan, M. B.; Hernandez, O.; Tomer, K. B.; Vestal, C. H.; Sanders, J. M.; Bend, J. R. Analysis of glutathione conjugates and related compounds by thermospray mass spectrometry. Biomedical \& Environmental Mass Spectrometry 1988, 15 (11), 623-633.

94. Baillie, T. A.; Davis, M. R. Mass spectrometry in the analysis of glutathione conjugates. Biological Mass Spectrometry 1993, 22 (6), 319-325.

95. Ramaley, C.; Leonard, S. C.; Miller, J. D.; Wilson, D. T.-M.; Chang, S. Y.; Chen, Q.; Li, F.; Du, C. In vitro metabolism of 3, 4methylenedioxymethamphetamine in human hepatocytes. Journal of Analytical Toxicology 2014, 38 (5), 249-255.

96. Wood, W. G.; Weatherall, D. J. Haemoglobin synthesis during human foetal development. Nature 1973, 244 (5412), 162. 
97. Konigsberg, W.; Hill, R. J. The structure of human hemoglobin III. the sequence of amino acids in the tryptic peptides of the alpha chain. Journal of Biological Chemistry 1962, 237 (8), 2547-2561.

98. Goldstein, J.; Konigsberg, W.; Hill, R. J. The structure of human hemoglobin $\mathrm{VI}$. the sequence of amino acids in the tryptic peptides of the beta chain. Journal of Biological Chemistry 1963, 238 (6), 2016-2027.

99. Blacken, G. R.; Wang, Y.; Lopez, J. A.; Fu, X. Cysteine 93 of hemoglobin beta chain is the major target of oxidation during red blood cell storage. 2009. Am Soc Hematology.

100. Kruse, A.; Uehlinger, D. E.; Gotch, F.; Kotanko, P.; Levin, N. W. Red blood cell lifespan, erythropoiesis and hemoglobin control. In Hemodialysis-From Basic Research to Clinical Trials, 161 ed.; Karger Publishers: 2008; pp 247-254.

101. Hinderling, P. H. Red blood cells: A neglected compartment in pharmacokinetics and pharmacodynamics. Pharmacological Reviews 1997, 49 (3), 279.

102. Reichel, C.; von Falkenhausen, M.; Brockmeier, D.; Dengler, H. J. Characterization of cyclosporine a uptake in human erythrocytes. European Journal of Clinical Pharmacology 1994, 46 (5), 417-419.

103. Lin, J. H.; Lin, T. H.; Cheng, H. Uptake and stereoselective binding of the enantiomers of MK-927, a potent carbonic anhydrase inhibitor, by human erythrocytes in vitro. Pharmaceutical Research 1992, 9 (3), 339-344.

104. Ferrari, V.; Cutler, D. J. Uptake of chloroquine by human erythrocytes. Biochemical Pharmacology 1990, 39 (4), 753-762.

105. Deuticke, B. Properties and structural basis of simple diffusion pathways in the erythrocyte membrane. In Reviews of Physiology, Biochemistry and Pharmacology, Volume 78, Springer: 1977; pp 197.

106. Sabbioni, G.; Neumann, H. G. Biomonitoring of arylamines: hemoglobin adducts of urea and carbamate pestides. Carcinogenesis 1990, 11 (1), 111-115.

107. Sabbioni, G. Hemoglobin binding of monocyclic aromatic amines: molecular dosimetry and quantitative structure activity relationships for the N-oxidation. Chemico-Biological Interactions 1992, 81 (1-2), $91-117$. 
108. McClure, G. Y.; Freeman, J. P.; Lay, J. O.; Hinson, J. A. Haemoglobin adducts as biomarkers of exposure to the herbicides propanil and fluometuron. Biomarkers 1996, 1 (2), 136-140.

109. Noort, D.; Fidder, A.; Degenhardt-Langelaan, C. E. A. M.; Hulst, A. G. Retrospective detection of sulfur mustard exposure by mass spectrometric analysis of adducts to albumin and hemoglobin: an in vivo study. Journal of Analytical Toxicology 2008, 32 (1), 25-30.

110. Dieckhaus, C. M.; Fernandez-Metzler, C. L.; King, R.; Krolikowski, P. H.; Baillie, T. A. Negative ion tandem mass spectrometry for the detection of glutathione conjugates. Chemical Research in Toxicology 2005, 18 (4), 630-638.

111. Beck, S.; Michalski, A.; Raether, O.; Lubeck, M.; Kaspar, S.; Goedecke, N.; Baessmann, C.; Hornburg, D.; Meier, F.; Paron, I.; Kulak, N. A.; Cox, J.; Mann, M. The Impact II, a very high-resolution quadrupole time-of-flight instrument (QTOF) for deep shotgun proteomics. Molecular \& Cellular Proteomics : MCP 2015, 14 (7), 2014-2029.

112. de Hoffman, E.; Stroobant, V. Mass Analyzers. In Mass Spectrometry: Principles and Applications, 3 ed.; John Wiley \& Sons: West Sussex, 2007; pp 85-174.

113. Switzar, L.; Giera, M.; Niessen, W. M. A. Protein digestion: An overview of the available techniques and recent developments. Journal of Proteome Research 2013, 12 (3), 1067-1077.

114. Burkhart, J. M.; Schumbrutzki, C.; Wortelkamp, S.; Sickmann, A.; Zahedi, R. P. Systematic and quantitative comparison of digest efficiency and specificity reveals the impact of trypsin quality on MS-based proteomics. Journal of Proteomics 2012, 75 (4), 1454-1462.

115. Navas, J. M.; Segner, H. In-vitro screening of the antiestrogenic activity of chemicals. Expert Opinion on Drug Metabolism \& Toxicology 2008, 4 (5), 605-617.

116. Attia, S. M. Deleterious effects of reactive metabolites. Oxidative Medicine and Cellular Longevity 2010, 3 (4), 238-253.

117. Miller, J. A.; Miller, E. C. Metabolism of drugs in relation to carcinogenicity. Annals of the New York Academy of Sciences 1965, 123 (1), 125140. 
118. Ikehata, K.; Duzhak, T. G.; Galeva, N. A.; Ji, T.; Koen, Y. M.; Hanzlik, R. P. Protein targets of reactive metabolites of thiobenzamide in rat liver in vivo. Chemical Research in Toxicology 2008, 21 (7), 14321442.

119. Lu, D.; Sullivan, M. M.; Phillips, M. B.; Peterson, L. A. Degraded protein adducts of cis-2-butene-1,4-dial are urinary and hepatocyte metabolites of furan. Chemical Research in Toxicology 2009, 22 (6), 997-1007.

120. Thompson, R. A.; Isin, E. M.; Li, Y.; Weaver, R.; Weidolf, L.; Wilson, I.; Claesson, A.; Page, K.; Dolgos, H.; Kenna, J. G. Risk assessment and mitigation strategies for reactive metabolites in drug discovery and development. Chemico-Biological Interactions 2011, 192 (1-2), 65-71.

121. Meneses-Lorente, G.; Sakatis, M. Z.; Schulz-Utermoehl, T.; Nardi, C. D.; Watt, A. P. A quantitative high-throughput trapping assay as a measurement of potential for bioactivation. Analytical Biochemistry 2006, 351 (2), 266-272.

122. Schadt, S.; Simon, S.; Kustermann, S.; Boess, F.; McGinnis, C.; Brink, A.; Lieven, R.; Fowler, S.; Youdim, K.; Ullah, M.; Marschmann, M.; Zihlmann, C.; Siegrist, Y. M.; Cascais, A. C.; Di Lenarda, E.; Durr, E.; Schaub, N.; Ang, X.; Starke, V.; Singer, T.; Alvarez-Sanchez, R.; Roth, A. B.; Schuler, F.; Funk, C. Minimizing DILI risk in drug discovery - A screening tool for drug candidates. Toxicology in Vitro 2015, 30 (1, Part B), 429-437.

123. Hoffmann, K. J.; Streeter, A. J.; Axworthy, D. B.; Baillie, T. A. Structural characterization of the major covalent adduct formed in vitro between acetaminophen and bovine serum albumin. ChemicoBiological Interactions 1985, 53, 155-172.

124. James, L. P.; Letzig, L.; Simpson, P. M.; Capparelli, E.; Roberts, D. W.; Hinson, J. A.; Davern, T. J.; Lee, W. M. Pharmacokinetics of acetaminophen-protein adducts in adults with acetaminophen overdose and acute liver failure. Drug Metabolism and Disposition 2009, 37 (8), 1779-1784.

125. McGill, M. R.; Lebofsky, M.; Norris, H. R.; Slawson, M. H.; Bajt, M. L.; Xie, Y.; Williams, C. D.; Wilkins, D. G.; Rollins, D. E.; Jaeschke, H. Plasma and liver acetaminophen-protein adduct levels in mice after acetaminophen treatment: Dose - response, mechanisms, and clinical implications. Toxicology and Applied Pharmacology 2013, 269 (3), 240-249. 
126. Zheng, J.; Ma, L.; Xin, B.; Olah, T.; Humphreys, W. G.; Zhu, M. Screening and identification of GSH-trapped reactive metabolites using hybrid triple quadruple linear ion trap mass spectrometry. Chemical Research in Toxicology 2007, 20 (5), 757-766.

127. Zhu, X.; Kalyanaraman, N.; Subramanian, R. Enhanced screening of glutathione-trapped reactive metabolites by in-source collisioninduced dissociation and extraction of product ion using UHPLChigh resolution mass spectrometry. Analytical Chemistry 2011, 83 (24), 9516-9523.

128. Ma, L.; Wen, B.; Ruan, Q.; Zhu, M. Rapid screening of glutathione-trapped reactive metabolites by linear ion trap mass spectrometry with isotope pattern-dependent scanning and postacquisition data mining. Chemical Research in Toxicology 2008, 21 (7), 1477-1483.

129. Schaber, G.; Stevens, I.; Gaertner, H. J.; Dietz, K.; Breyer-Pfaff, U. Pharmacokinetics of clozapine and its metabolites in psychiatric patients: plasma protein binding and renal clearance. British Journal of Clinical Pharmacology 1998, 46 (5), 453-459.

130. Sergi, M.; Bafile, E.; Compagnone, D.; Curini, R.; DGÇÖascenzo, G.; Romolo, F. S. Multiclass analysis of illicit drugs in plasma and oral fluids by LC-MS/MS. Analytical and Bioanalytical Chemistry 2009, 393 (2), 709-718.

131. Dain, J. G.; Nicoletti, J.; Ballard, F. Biotransformation of clozapine in humans. Drug Metabolism and Disposition 1997, 25 (5), 603-609.

132. Volpicelli, S. A.; Centorrino, F.; Puopolo, P. R.; Kando, J.; Frankenburg, F. R.; Baldessarini, R. J.; Flood, J. G. Determination of clozapine, norclozapine, and clozapine-N-oxide in serum by liquid chromatography. Clinical Chemistry 1993, 39 (8), 1656-1659.

133. de Almeida, C. A. A.; Oliveira, M. c. S.; Mallmann, C. A.; Martins, A. F. Determination of the psychoactive drugs carbamazepine and diazepam in hospital effluent and identification of their metabolites. Environmental Science and Pollution Research 2015, 22 (21), 17192-17201.

134. Yamada, H.; Ishii, Y.; Oguri, K. Metabolism of drugs of abuse: its contribution to the toxicity and the inter-individual differences in drug sensitivity. Journal of Health Science 2005, 51 (1), 1-7. 
135. Dragovic, S.; Gunness, P.; Ingelman-Sundberg, M.; Vermeulen, N. P. E.; Commandeur, J. N. M. Characterization of human cytochrome P450s involved in the bioactivation of clozapine. Drug Metabolism and Disposition 2013, 41 (3), 651.

136. Li, Y.; Wu, L.; Gu, Y.; Si, D.; Liu, C. Metabolism of aildenafil in vivo in rats and in vitro in mouse, rat, dog, and human liver microsomes. Drug Testing and Analysis 2014, 6 (6), 552-562.

137. Moller, C.; Davis, W. C.; Thompson, V. R.; Mari, F.; DeCaprio, A. P. Proteomic analysis of thiol modifications and assessment of structural changes in hemoglobin induced by the aniline metabolites $\mathrm{N}$-phenylhydroxylamine and nitrosobenzene. Scientific Reports 2017, 7 (1), 14794.

138. Axworthy, D. B.; Hoffmann, K. J.; Streeter, A. J.; Calleman, C. J.; Pascoe, G. A.; Baillie, T. A. Covalent binding of acetaminophen to mouse hemoglobin. Identification of major and minor adducts formed in vivo and implications for the nature of the arylating metabolites. Chemico-Biological Interactions 1988, 68 (1-2), 99-116.

139. Agilent Technologies . Mass Accuracy and Mass Resolution in TOF MS. 2011.

140. Day, B. W.; Doxtader, M. M.; Rich, R. H.; Skipper, P. L.; Singh, K.; Dasari, R. R.; Tannenbaum, S. R. Human serum albumin-benzo [a] pyrene anti-diol epoxide adduct structure elucidation by fluorescence line narrowing spectroscopy. Chemical Research in Toxicology 1992, 5 (1), 71-76.

141. Dooley, G. P.; Hanneman, W. H.; Carbone, D. L.; Legare, M. E.; Andersen, M. E.; Tessari, J. D. Development of an immunochemical detection method for atrazine-induced albumin adducts. Chemical Research in Toxicology 2007, 20 (7), 10611066.

142. Obach, R. S.; Kalgutkar, A. S.; Soglia, J. R.; Zhao, S. X. Can in vitro metabolism-dependent covalent binding data in liver microsomes distinguish hepatotoxic from nonhepatotoxic drugs? An analysis of 18 drugs with consideration of intrinsic clearance and daily dose. Chemical Research in Toxicology 2008, 21 (9), 1814-1822. 
143. Rubino, F. M.; Pitton, M.; Di Fabio, D.; Colombi, A. Toward an omic physiopathology of reactive chemicals: thirty years of mass spectrometric study of the protein adducts with endogenous and xenobiotic compounds. Mass Spectrometry Reviews 2009, 28 (5), 725-784. 


\section{APPENDIX}

\section{Appendix 1. Precursor ion scan TIC and XIC data for the drugs of abuse.}
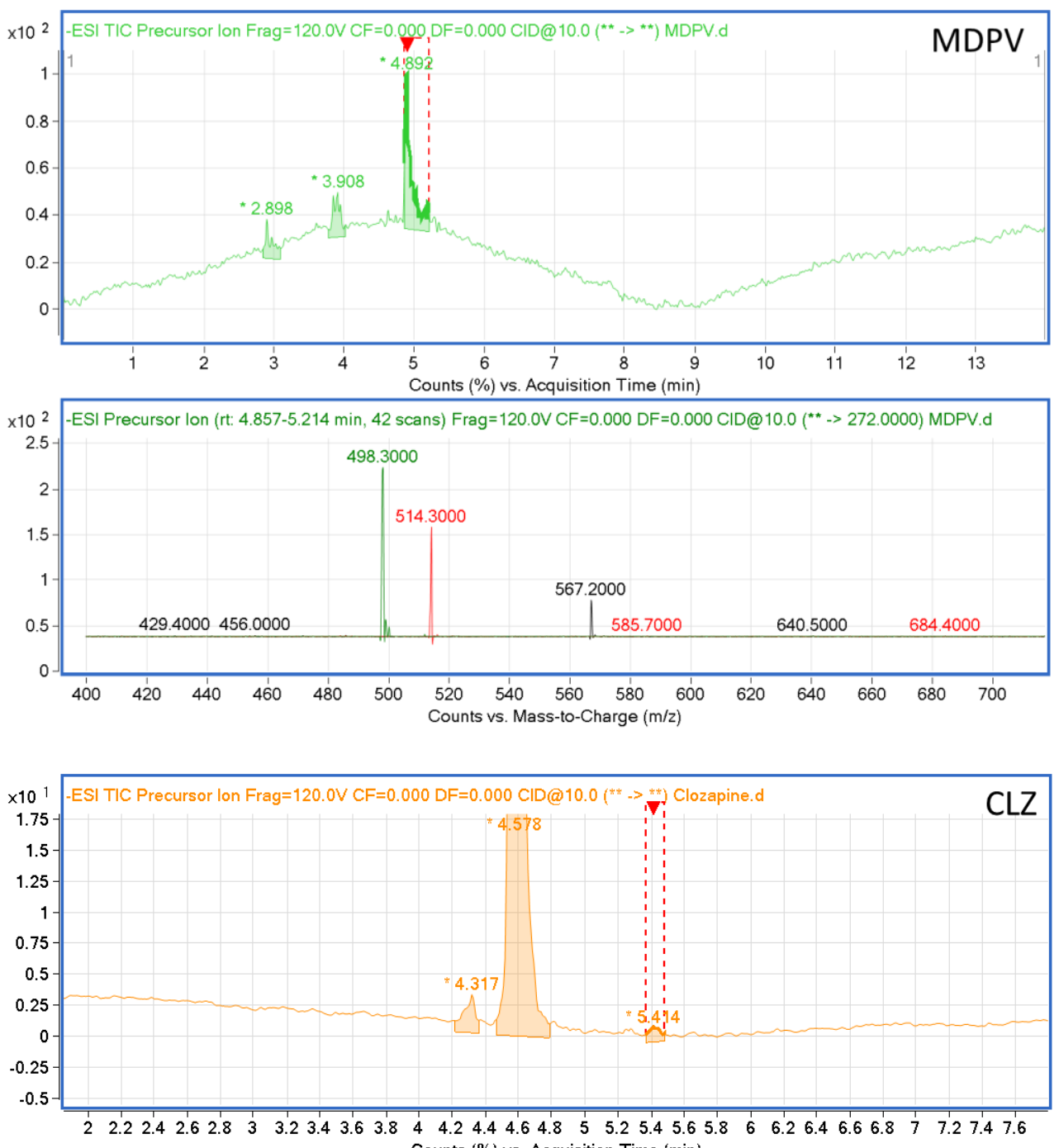

Counts (\%) vs. Acquisition Time (min)

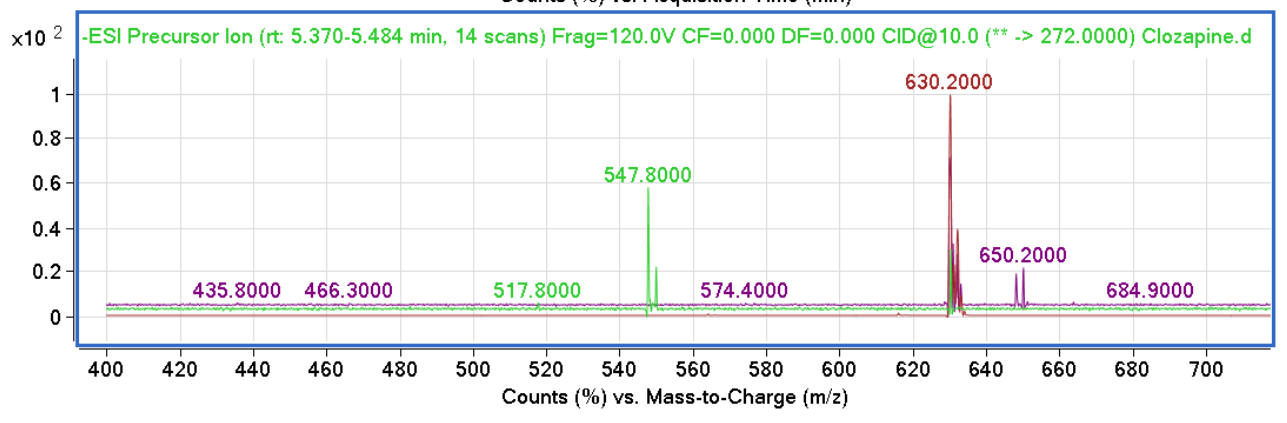



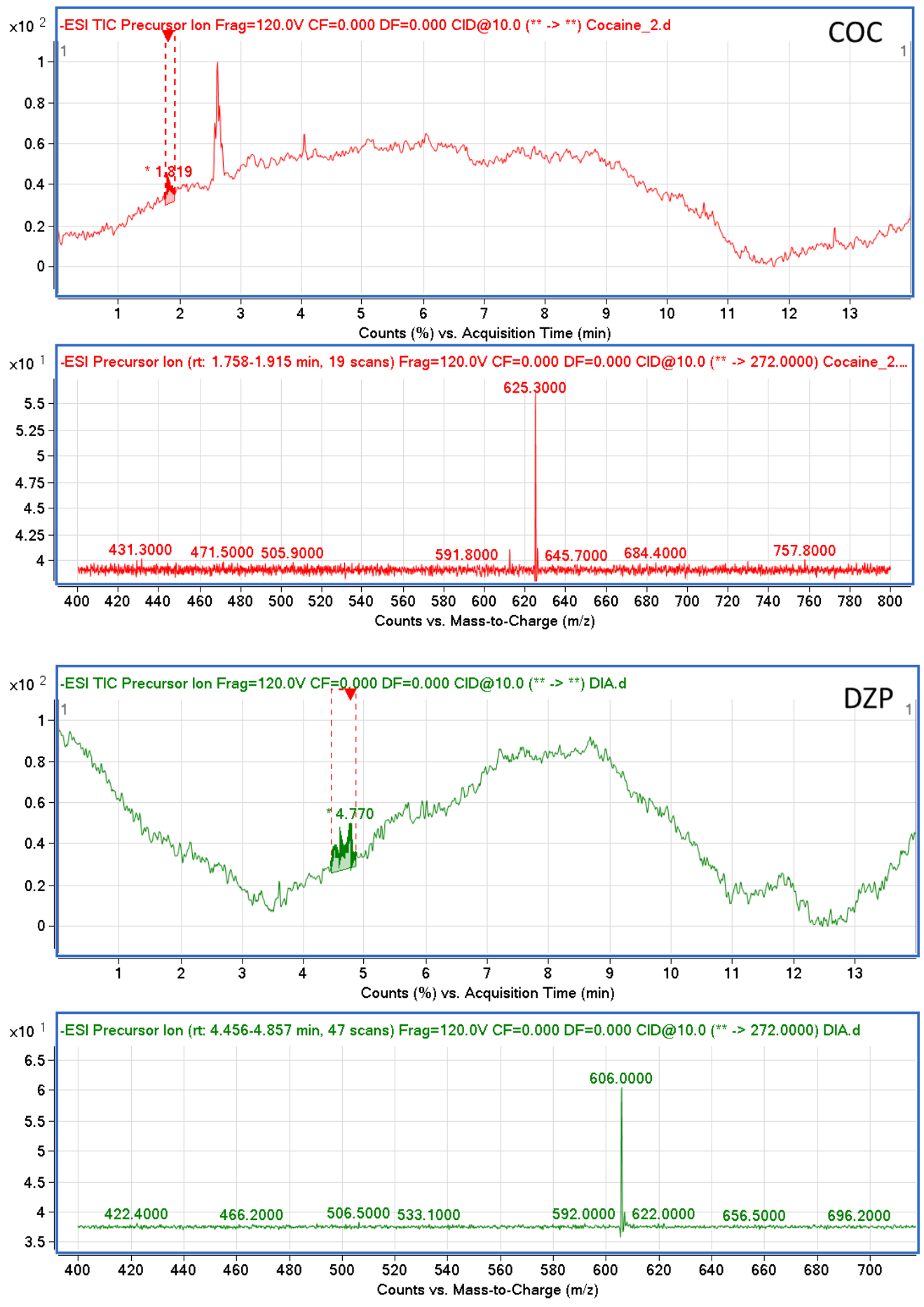

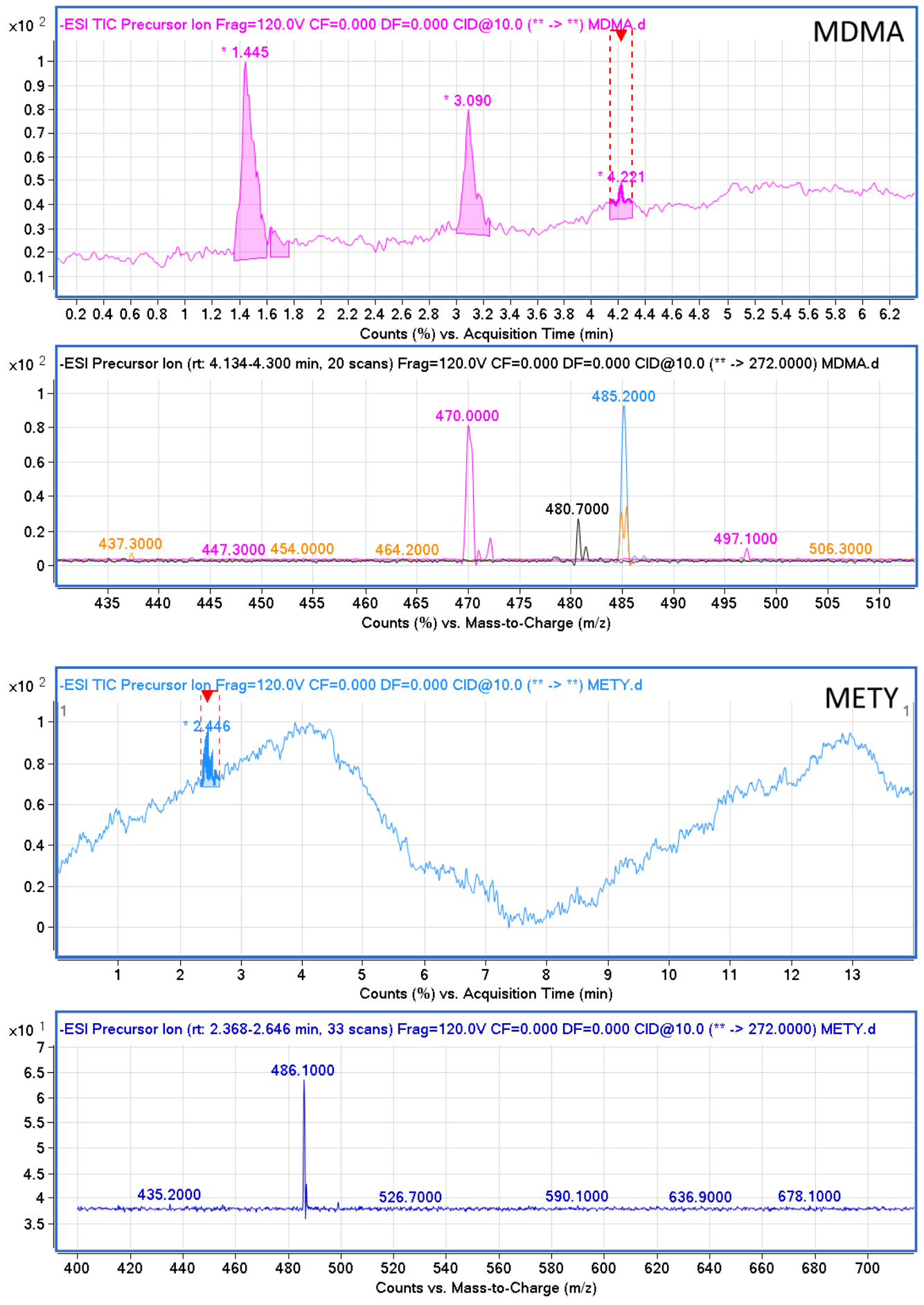

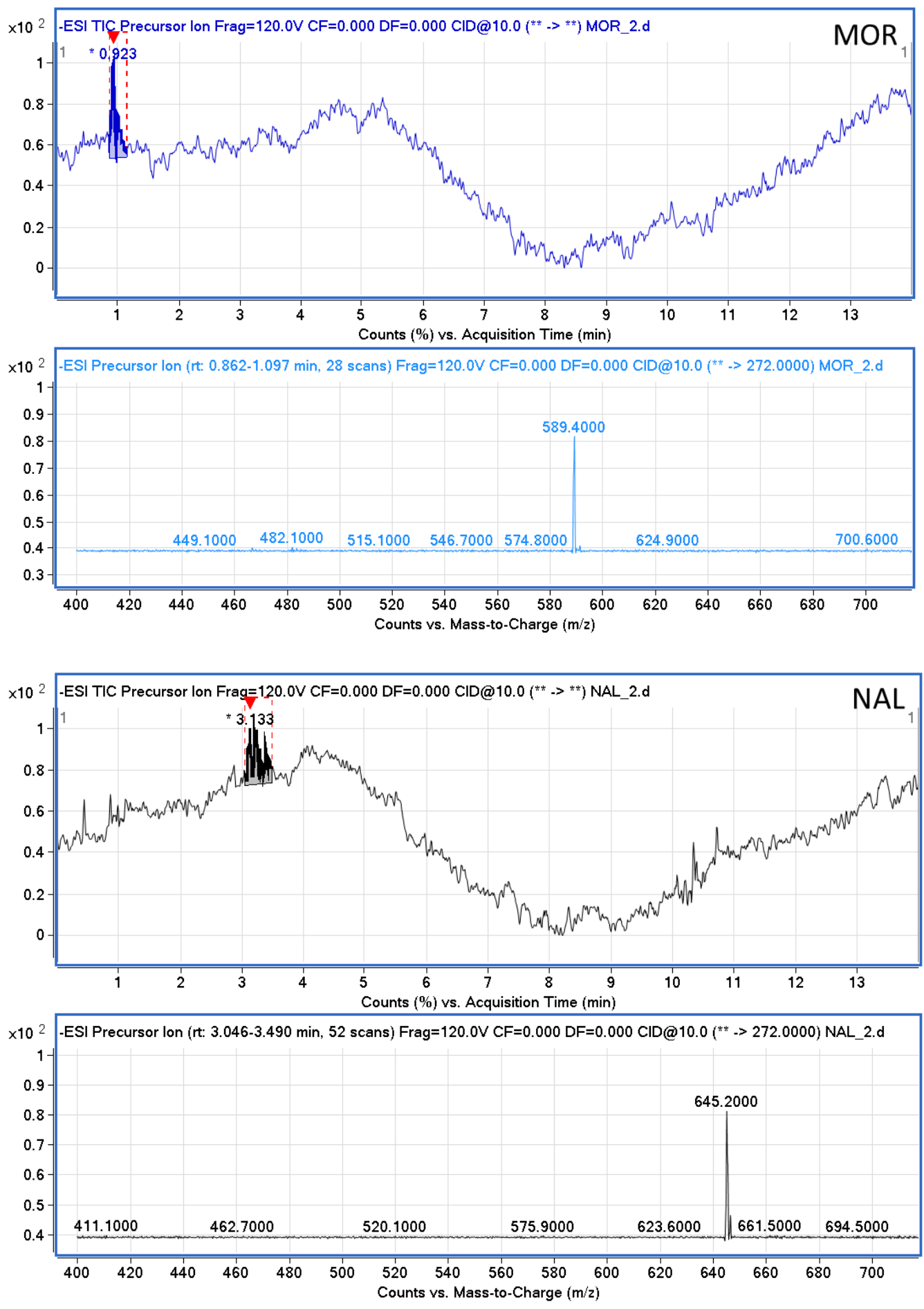

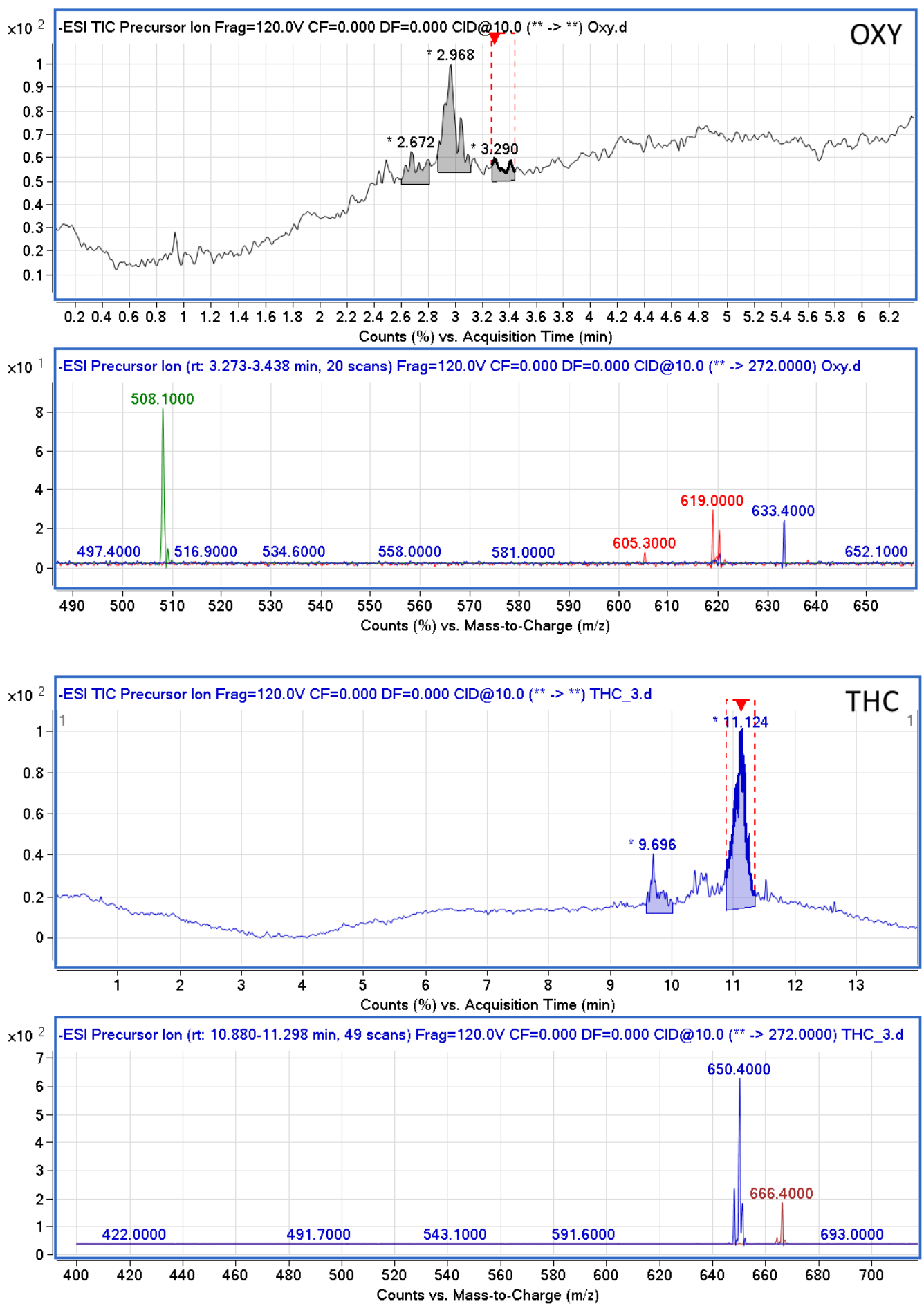
VITA

\title{
R. ALLEN GILLILAND
}

\author{
2009 - $2013 \quad$ Bachelor's Degree in Chemistry \\ Minors in biochemistry and forensic science \\ New Mexico State University \\ Las Cruces, NM \\ 2011 - $2013 \quad$ Undergraduate Research Assistant \\ New Mexico State University \\ Advisor: Jeffrey Arterburn \\ Las Cruces, NM \\ $2013-2016 \quad$ Teaching Assistant \\ Florida International University \\ Miami, FL \\ $2013-2016 \quad$ Master's of Science in Forensic Science \\ Florida International University \\ Earned en route \\ Advisor: Anthony DeCaprio \\ Miami, FL \\ $2013-2018 \quad$ Doctoral Candidate, Forensic Chemistry \\ Florida International University \\ Advisor: Anthony DeCaprio \\ Miami, FL \\ $2017-2018 \quad$ Teaching Assistant \\ Florida International University \\ Miami, FL
}

\section{PUBLICATIONS AND PRESENTATIONS}

Nashir, A.T.M.; Gilliland, R.A.; Arterburn, J.B. Regioselective control of the cycloaddition reaction of vinylboronic acid derivatives with tetrazines for the synthesis of pyridazines. (2012) Poster presentation Southwest Regional Meeting of the American Chemical Society. Baton Rogue, LA.

Nashir, A.T.M.; Gilliland, R.A.; Arterburn, J.B. Regioselective control of the cycloaddition reaction of vinylboronic acid derivatives with tetrazines for the synthesis of pyridazines. (2013) Poster presentation at the Undergraduate Research and Creative Arts Symposium. Las Cruces, NM. 
Gilliland, R.A.; Moller, C.; DeCaprio, A.P. LC-MS/MS based analysis of in vitro covalent modifications of glutathione and peptide thiols by drugs of abuse. (2017) Poster presentation at the American Academy of Forensic Sciences Annual Meeting. New Orleans, LA.

Gilliland, R.A.; Moller, C.; DeCaprio, A.P. LC-MS/MS based analysis of in vitro covalent modifications of glutathione and peptide thiols by drugs of abuse. (2017) Poster presentation at the Graduate Student Appreciation Week Scholarly Forum. Miami, FL.

Gilliland, R.A.; Moller, C.; DeCaprio, A.P. LC-MS/MS based analysis of in vitro covalent modifications of glutathione and peptide thiols by drugs of abuse. (2017) Poster presentation at the International Forensic Research Institute Symposium. Miami, FL.

Gilliland, R.A. and DeCaprio, A.P. A mass spectrometric approach to the analysis of covalent modifications of blood proteins by drugs of abuse. (2018) Poster presentation at the American Academy of Forensic Sciences Annual Meeting. Seattle, WA. Awarded First Place Toxicology Poster Presentation.

Gilliland, R.A. and DeCaprio, A.P. Analysis and characterization of in vitro glutathione adducts formed with drugs of abuse. (2018) Poster presentation at the Pittsburgh Conference and Expo. Orlando, FL.

Gilliland, R.A. and DeCaprio, A.P. Analysis of drug-protein modifications in forensic toxicology. (2018) Oral presentation at the Pittsburgh Conference and Expo. Orlando, FL.

Moller, C.; Gilliland, R.A.; DeCaprio, A.P. In vitro peptide-based assay for the detection of thiol adducts of drugs of abuse. (2018) To be submitted to Bioanalysis. (in preparation)

Gilliland, R.A.; Moller, C.; DeCaprio, A.P. LC-MS/MS based detection and characterization of covalent glutathione modifications formed by reactive drug of abuse metabolites. (2018) Xenobiotica. (accepted) 